\title{
Experimentelle Untersuchungen zur reaktiven Streuung laserangeregter Teilchen
}

\author{
Dissertation \\ zur Erlangung des Doktorgrades \\ der Mathematisch-Naturwissenschaftlichen Fakultäten \\ der Georg-August-Universität zu Göttingen
}

vorgelegt von

Matthias Färber

aus Hannover

Göttingen 1997 
D7

Referent:

Prof. Dr. H. Pauly

Korreferent:

Prof. Dr. F. Smend

Tag der mündlichen Prüfung: 19. 6. 1997 


\section{Inhaltsverzeichnis}

1 Einleitung \& Übersicht

2 Experimentelle Grundlagen 5

2.1 Überblick über den Aufbau . . . . . . . . . . . . . . . . . . . . . . 5

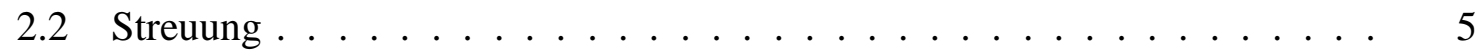

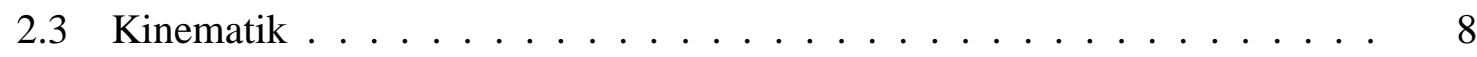

2.4 Anregung von Natrium $3^{2} S_{1 / 2} \rightarrow 3^{2} P_{3 / 2} \ldots \ldots \ldots \ldots \ldots$

2.5 Düsenstrahlen . . . . . . . . . . . . . . . . . . . . . 11

2.6 Pseudostatistische Laufzeitanalyse . . . . . . . . . . . . . . . . 13

3 Experimentelle Realisation $\quad 17$

3.1 Vakuumapparatur . . . . . . . . . . . . . . . . . . . . . 17

3.2 Molekularstrahlquellen . . . . . . . . . . . . . . . . . . . . . . . 19

3.3 Lasersystem . . . . . . . . . . . . . . . . . . . . . . . 21

3.4 Anregung des Natriums . . . . . . . . . . . . . . . . . . . . . . 26

3.5 Detektoren . . . . . . . . . . . . . . . . . . . . . 28

3.6 Pseudostatistische Modulation . . . . . . . . . . . . . . . . . . 31

3.7 Meßdatenaufnahme und -verarbeitung . . . . . . . . . . . . 33

4 Eigenschaften der verwendeten Apparatur 35

4.1 Anregung im Natriumstrahl . . . . . . . . . . . . . . . . . . 35

4.1 .1 Effizienz der Anregung . . . . . . . . . . . . . . . . 35

4.1.2 Methoden der Bestimmung von $\eta \ldots \ldots \ldots \ldots$

4.1 .3 Eigene Ergebnisse . . . . . . . . . . . . . . 36

4.2 Auswertung der Flugzeitspektren . . . . . . . . . . . . . . . . . . . 40

4.2 .1 Typische Spektren . . . . . . . . . . . . . . . . . . 40

4.2 .2 Anpassung von Flugzeitverteilungen . . . . . . . . . . . . 40

4.2.3 Extraktion der Querschnitte . . . . . . . . . . . . . . . . 41

4.3 Kinematik und Auflösungsvermögen . . . . . . . . . . . . . . . . . . 42

4.3.1 Transformation vom Labor- ins Schwerpunktsystem . . . . . . . 42

4.3.2 Behandlung von Mittelungseffekten durch Simulation _. . . . . 43

4.3.3 Testmessungen am System $\mathrm{Na}-\mathrm{Xe} \ldots \ldots \ldots \ldots$. . . . . . 45

4.3.4 Auswirkungen auf das Auflösungsvermögen $\ldots \ldots \ldots \ldots$ 
$5 \quad$ Streuung von $\mathrm{SF}_{6}$ an $\mathrm{Na}(3 \mathrm{~S})$ und $\mathrm{Na}(3 \mathrm{P}) \quad \mathbf{5 3}$

5.1 Überblick . . . . . . . . . . . . . . . . . . . . . . 53

5.2 Untersuchte Fragestellungen . . . . . . . . . . . . . . . . 53

5.3 Experimentelle Bedingungen . . . . . . . . . . . . . 55

5.4 Experimentelle Ergebnisse . . . . . . . . . . . . . . . . . 56

5.5 Interpretation der Ergebnisse . . . . . . . . . . . . 76

6 Streuung von $\mathrm{SiF}_{4}$ an $\mathrm{Na}(3 \mathrm{~S})$ und $\mathrm{Na}(3 \mathrm{P}) \quad 83$

6.1 Überblick . . . . . . . . . . . . . . . . . . . . . . 83

6.2 Experimentelle Ergebnisse . . . . . . . . . . . . . . . . . . . 84

6.3 Statistische Interpretation der Meßdaten . . . . . . . . . . . . . . . 89

6.4 Vergleich mit anderen Experimenten . . . . . . . . . . . . . 90

7 Streuung von $\mathrm{NH}_{3}$ an $\mathrm{Na}(3 \mathrm{~S})$ und $\mathrm{Na}(3 \mathrm{P}) \quad 95$

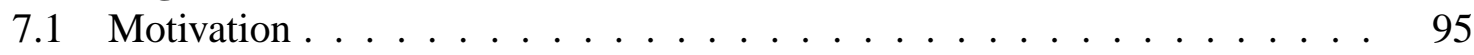

7.2 Mögliche Ausgangskanäle . . . . . . . . . . . . . . . . 95

7.3 Experimentelle Bedingungen . . . . . . . . . . . . . . . . . . 96

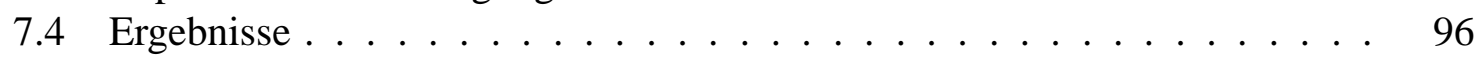

8 Zusammenfassung $\quad 103$

$\begin{array}{llr}\text { A } & \text { Eingesetzte Software } & 117\end{array}$ 


\section{Kapitel 1}

\section{Einleitung \& Übersicht}

Das Interesse dieser Arbeit gilt der experimentellen Untersuchung von Stoßprozessen zwischen elektronisch angeregtem Natrium und mehratomigen Molekülen. Bei der Streuung elektronisch angeregter Spezies sind grundsätzlich mehrere Potentialflächen involviert, deren Symmetrie- und sonstige Eigenschaften sich unterscheiden. Besondere Verhältnisse ergeben sich dadurch, daß im Stoß ein nicht-adiabatischer Übergang zwischen diesen Potentialflächen möglich ist. Ein solcher Übergang kann sehr verschiedene Auswirkungen auf den Streuprozess haben :

- Zunächst wird dem Streusystem zusätzliche Energie zugeführt, wenn ein Übergang auf eine tieferliegende Potentialfläche erfolgt. Für einige Systeme kann so eine im elektronischen Grundzustand vorhandene energetische Barriere überwunden werden.

- Über die reine Energiezufuhr hinaus ist die Struktur der Potentialflächen für Reaktivität und Reaktionsverhalten entscheidend. So findet eine Reaktion von Natrium mit $\mathrm{O}_{2}$ nur in einem speziellen Anregungszustand des Natriums (4D) statt, obwohl die Reaktion auch für andere Anregungszustände energetisch möglich erscheint [53]. Ein Überblick über reaktive Prozesse mit angeregten Atomen ist in [29] gegeben.

- Auch ohne chemische Reaktion kann der nicht-adiabatische Übergang den Streuprozess beeinflussen. In diesem Fall findet eine Übertragung der Energie in die zugänglichen Freiheitsgrade der Translation, Rotation und Vibration statt. Diese Art von Prozeß wurde schon in den Anfangszeiten der Quantenmechanik an der Löschung der Fluoreszenz von Alkalien durch Puffergase beobachtet [50].

Die ideale experimentelle Untersuchungsmethode dieser nicht-adiabatischen Prozesse ist die vollständig zustandsselektive Streuung, d. h.

1. vor dem Stoß wird eine Präparation der Zustände der Stoßpartner so vorgenommen, daß diese Zustände exakt bekannt sind,

2. die Streuung findet unter Einzelstoßbedingungen statt,

3. nach dem Stoß werden die gestreuten Teilchen auf ihren Quantenzustand hin analysiert. 
Punkt 1 ist für spezielle Systeme zu erreichen, indem Moleküle im Düsenstrahl expandiert werden, so daß für Vibration und Rotation idealerweise nur noch der Grundzustand besetzt ist. Mit infraroter, intensiver Laserstrahlung können - insbesondere für $\mathrm{SF}_{6}$ [55] spezielle Vibrationsniveaus bevölkert werden; die selektive Anregung einzelner elektronischer Anregungszustände ist insbesondere bei Atomen durch schmalbandige Laserstrahlung möglich. Punkt 2 ist mit dem Stand der Technik entsprechenden Molekularstrahl-Streuapparaturen problemlos erreichbar. Punkt 3 stellt das größte Problem dar; für spezielle Spezies ist die zustandsspezifische Detektion mit spektroskopischen Methoden möglich. Als Standardverfahren für alle Systeme erlaubt die - auch für diese Arbeit verwendete - Messung der Translationsenergie der gestreuten Teilchen immerhin, die Aufteilung in Translations- und innere Energie zu bestimmen. Unter günstigen Umständen sind daraus Rückschlüsse auf die Zustandsverteilung möglich.

Die theoretische Behandlung der nicht-adiabatischen Übergänge ist sehr schwierig. Zunächst müssen mit quantenchemischen Methoden die zugrundeliegenden Potentialflächen gewonnen werden. Dies ist für vielatomige Systeme noch recht aufwendig. Dann müssen Rechnungen der Quantendynamik oder entsprechende Trajektorienrechnungen [63] ausgeführt werden. Daraus ergibt sich eine „Arbeitsteilung“ zwischen experimentellen und theoretischen Arbeiten zur Aufklärung der Vorgänge bei nicht-adiabatischen Übergängen. Motivation für Arbeiten auf diesem Feld ist die Bedeutung dieser Vorgänge für ein grundlegendes Verständnis von Reaktionsdynamik.

Auch die oben beschriebenen experimentellen Untersuchungsmethoden sind von hohem technischem Aufwand begleitet. Das Hauptziel dieser Arbeit ist dementsprechend, die erzielten experimentellen Ergebnisse möglichst nachvollziehbar darzustellen.

In Kapitel 2 soll zum einen eine Klassifikation von Streuprozessen vorgenommen werden. Zum anderen werden die physikalischen Gesetzmäßigkeiten, die zum Verständnis der eingesetzten experimentellen Techniken und Auswertungsrechnungen erforderlich sind, dargestellt.

Kapitel 3 widmet sich detailliert der Beschreibung der Gerätschaften und des Apparaturbetriebes.

Die für das Verständnis der Ergebnisse wichtigen Eigenschaften der Apparatur sowie die grundsätzlich zu erhaltenden Meßergebnisse und deren Auswertung werden in Kapitel 4, gemeinsam mit Testmessungen elastischer Streuung, dargestellt.

Die folgenden Kapitel enthalten die Beschreibung der Spezifika, Ergebnisse und Interpretation für die untersuchten Systeme $\mathrm{Na}(3 \mathrm{~S}) / \mathrm{Na}(3 \mathrm{P})-\mathrm{SF}_{6}, \mathrm{Na}(3 \mathrm{~S}) / \mathrm{Na}(3 \mathrm{P})-\mathrm{SiF}_{4}$ und $\mathrm{Na}(3 \mathrm{~S}) / \mathrm{Na}(3 \mathrm{P})-$ $\mathrm{NH}_{3}$. 


\section{Kapitel 2}

\section{Experimentelle Grundlagen}

\section{1 Überblick über den Aufbau}

Zunächst sei ein grober Überblick über die Meßmethode gegeben; Abb. 2.1 zeigt die wesentlichen Bestandteile des Aufbaus in schematischer Form. Ein Natrium-Atomstrahl und ein Molekularstrahl sind unter $90^{\circ}$ gekreuzt. Der Nachweis der gestreuten Teilchen findet winkelabhängig in der Streuebene statt. Zur Anwendung kommen zwei Detektoren, die nach dem Prinzip der Oberflächenionisation (Langmuir-Taylor-Detektor) bzw. der Laserinduzierten Fluoreszenz arbeiten. Die Modulation des Natriumstrahls mit einer pseudostatistischen Sequenz erlaubt eine Flugzeitanalyse der gestreuten Teilchen. Mit Hilfe eines kontinuierlichen Farbstofflasers kann der Natriumstrahl auf der Natrium-D-Linie effizient angeregt werden. Durch die pseudostatistische Modulation des Laserstrahls kann die Flugzeitanalyse auch für die Streuung im angeregten Zustand erfolgen.

\subsection{Streuung}

\section{Definition der Meßgrößen}

Für die gegebene Anordnung ist die entscheidende Meßgröße der differentielle Streuquerschnitt

$$
\frac{d \sigma}{d \omega} \sim \frac{d N}{d \omega \cdot d t}
$$

also die pro Zeiteinheit in ein Winkelintervall $d \omega$ gestreute Teilchenzahl $d N$. Der in (2.1) fehlende Proportionalitätsfaktor enthält die Intensitäten der Strahlen; für eine absolute Kalibrierung müssen diese bekannt sein. Dann hat $\frac{d \sigma}{d \omega}$ die Einheit einer Fläche pro Winkelelement. Für den experimentell zugänglichen rechten Teil von 2.1 ergibt sich als Einheit Teilchen pro Sekunde. Integration über den gesamten Winkelbereich führt zum integralen oder totalen Streuquerschnitt

$$
\sigma=\int \frac{d \sigma}{d \omega} d \omega
$$




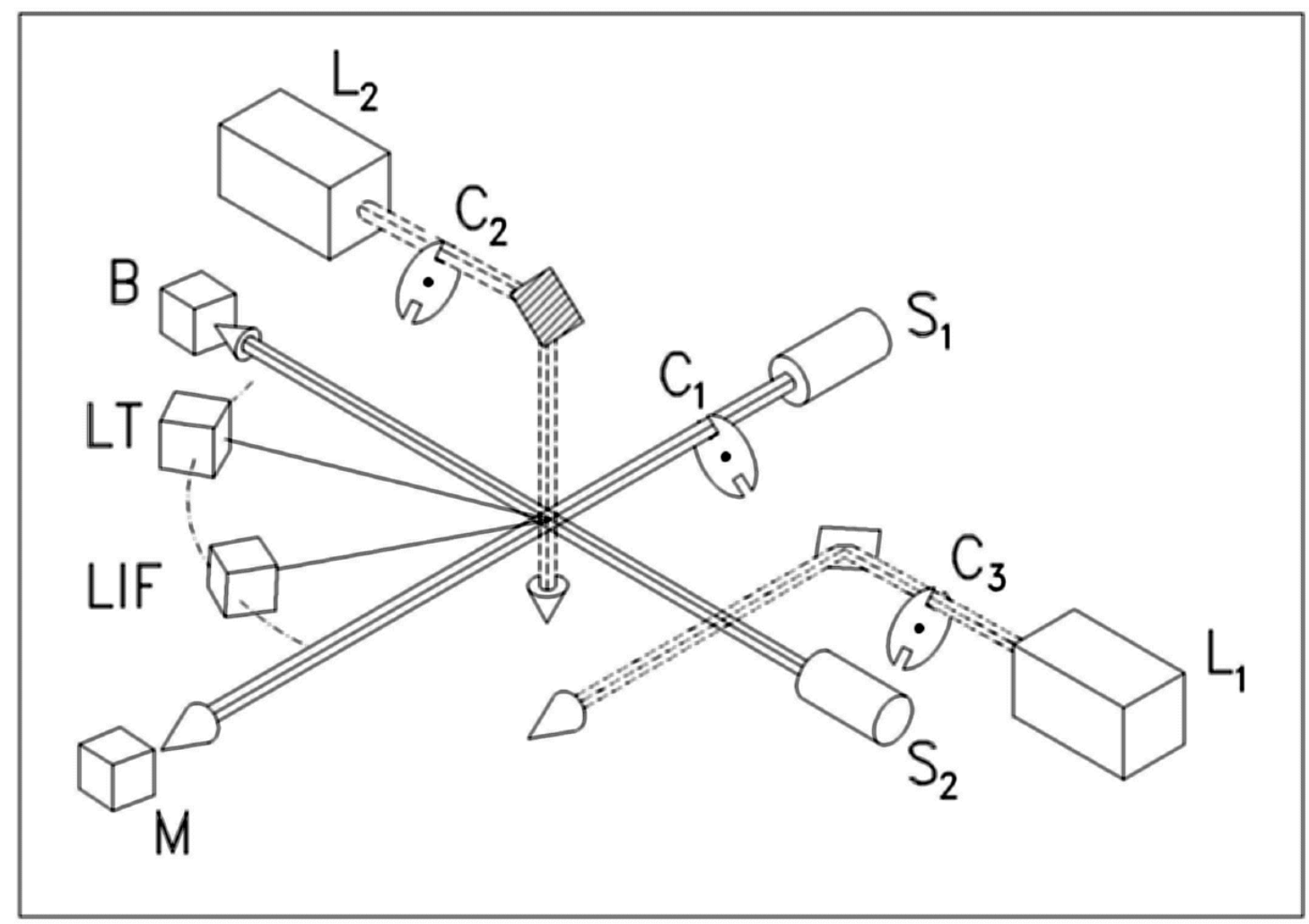

Abb. 2.1: Schema der Apparatur. Bedeutung der Beschriftung : $\mathrm{S}_{1,2}$ Natrium- bzw. Molekularstrahlquelle, $\mathrm{L}_{1} \mathrm{CO}_{2}$-Laser, $\mathrm{L}_{2}$ Farbstoff-Laser, $\mathrm{C}_{1-3}$ Chopper mit psudostatistischer Sequenz, B Bolometer, M Monitordetektor, LT und LIF Winkeldetektoren.

der, interpretiert im Modell harter Kugeln, die Fläche angibt, die ein Stoßpartner vom anderen wahrnimmt. Im allgemeinen ist der totale Streuquerschnitt ein Maß für die Wahrscheinlichkeit des Streuprozesses. Prinzipiell ist der totale Streuquerschnitt durch Integration einer gemessenen Winkelverteilung des differentiellen Querschnittes über den gesamten (Raum)Winkelbereich zu berechnen (Im folgenden ist mit „Winkelverteilung“ grundsätzlich die Winkelverteilung des differentiellen Querschnittes gemeint, soweit nicht anders spezifiziert). Allerdings läßt sich die Winkelverteilung meistens nicht für den gesamten Winkelbereich bestimmen; weiterhin ist auch hier eine absolute Kalibrierung der Intensitäten notwendig. Insofern ist eine Apparatur zur Messung differentieller Streuquerschnitte, wie sie hier verwendet wird, nicht optimal zur Bestimmung des totalen Querschnittes geeignet. Dennoch können aus dem Intensitätsvergleich der Winkelverteilungen zumindest qualitative Informationen über totale Querschnitte entnommen werden.

Das bisher gesagte gilt bei Streuexperimenten, die mehrere Ausgangskanäle haben (z. B. elastisch/reaktiv), für jeden Kanal einzeln, sofern sich die Kanäle experimentell trennen lassen.

Wird der differentielle Querschnitt zusätzlich noch in Abhängigkeit von der Laufzeit der gestreuten Teilchen gemessen, spricht man vom doppelt differentiellen Querschnitt 


$$
\frac{d \sigma}{d \omega \cdot d t} \sim \frac{d N}{d \omega \cdot d t^{2}}
$$

der die gestreute Intensität zusätzlich in Zeitintervalle $d t$ einordnet. Dementsprechend ergibt sich für die experimentelle Größe die Einheit $\frac{\text { Teilchen }}{s^{2}}$. Der differentielle Querschnitt, im folgenden zur Abgrenzung totaler differentieller Querschnitt genannt, wird durch Integration über die Zeit gewonnen. Aus der Laufzeit der Teilchen ergibt sich die kinetische Energie des Systems nach dem $S t o ß$ und damit $\frac{d \sigma^{2}}{d \omega d E}$.

Eine detaillierte Darstellung der Thematik findet sich z. B. in [8] oder [25].

\section{Zu unterscheidende Streuprozesse}

\section{Elastische Streuung}

Elastische Streuung findet dann statt, wenn die innere Energie der Stoßpartner sich im Stoß nicht ändert. Dies ist typischerweise der Fall für die Streuung zwischen zwei Atomen, wenn vom Energiebereich her die elektronischen Niveaus keine Rolle spielen. Im Stoß gilt also Energieerhaltung für die Translation; aufgrund dessen ist die Energie der gestreuten Teilchen mit der Stoßenergie festgelegt. Es gibt im Schwerpunktsystem (s. nächster Abschnitt) keine Änderung der Geschwindigkeit der Stoßpartner. Für ein solches System erbringt die Messung des totalen differentiellen Querschnittes schon die gesamte Information, die Messung doppelt differentieller Querschnitte hingegen redundante Information. Daher kann elastische Streuung für das beschriebene Experiment zur Eichung verwendet werden. Elastische Streumessungen finden ihre Bestimmung in der Erforschung des Wechselwirkungspotentials aus der Struktur der Winkelverteilung. Diese Messungen erfordern Experimente mit sehr guter Winkelauflösung.

\section{Inelastische Streuung}

Findet eine Änderung des Quantenzustandes (Vibrations-, Rotations- oder elektronischer Zustand) zumindest eines Stoßpartners statt, gilt nicht mehr wie im obigen Fall Erhaltung der Translationsenergie. Die maximale Information über ein solches System ist nur durch völlig zustandsselektive Messung doppelt differentieller Querschnitte möglich. Die Aufnahme nicht zustandsselektiver doppelt differentieller Querschnitte liefert im allgemeinen Fall immerhin die Summe und die Verteilung des Übertrages in die innere Energie. Die Auflösung in diskrete Niveaus ist in der Regel aufgrund mangelnder energetischer Auflösung oder fehlender Möglichkeit der Zuordnung zwischen energetisch ähnlichen Prozessen nicht möglich.

\section{Reaktive Streuung}

Im Falle eines reaktiven Stoßprozesses ändert sich auch noch die chemische Zusammensetzung der Stoßpartner. Zusätzlich zu den bei inelastischer Streuung zu gewinnenden Informationen ist hier noch eine nach der chemischen Spezies selektive Detektion erforderlich. Im Gegensatz zur inelastischen Streuung sind die Energieüberträge oft wesentlich größer (aufgrund von Endo-/Exothermizität). Infolgedessen ist es einfacher, Information durch doppelt 
differentielle Querschnitte zu gewinnen. Für die Selektion gilt das für inelastische Streuung gesagte.

Weiterhin liefert die Winkelverteilung Erkenntnisse über den Ablauf der Reaktion. Auf diesen Punkt wird in Kap. 5 eingegangen (siehe auch [48, 46, 2]).

\subsection{Kinematik}

Die physikalisch entscheidenden Vorgänge im Stoß äußern sich in der Verteilung der Intensitäten und Geschwindigkeiten der gestreuten Teilchen im Schwerpunktsystem. Da die Messung jedoch zwangsweise im System eines äußeren Betrachters, also im Laborsystem erfolgt, ist eine Transformation der Meßdaten erforderlich. Da bei dieser Transformation sich außer den Geschwindigkeiten auch die Intensitäten ändern, können Meßdaten erst nach der Transformation korrekt interpretiert werden. Für die Durchführung der Transformation sei der Vektorraum der Geschwindigkeiten betrachtet; im allgemeinen Fall ist eine dreidimensionale Betrachtung notwendig. Da hier nur Streuung in einer Ebene zu Diskussion steht, reicht eine zweidimensionale Betrachtung. Der Geschwindigkeitsraum kann in der Ebene anschaulich dargestellt werden; eine solche Darstellung wird Newton-Diagramm genannt. Abb. 2.2 zeigt ein solches Newton-Diagramm. Die Bedeutung der Größen ist

$\vec{v}_{1,2} \quad$ Geschwindigkeit von Primär- bzw. Sekundärteilchen vor dem Stoß im Laborsystem

$\vec{u}_{1,2} \quad$ Geschwindigkeit von Primär- bzw. Sekundärteilchen vor dem Stoß im Schwerpunktsystem

$\vec{v}_{1,2}^{\prime} \quad$ Geschwindigkeit von Primär- bzw. Sekundärteilchen nach dem Stoß im Laborsystem

$\vec{u}_{1,2}^{\prime} \quad$ Geschwindigkeit von Primär- bzw. Sekundärteilchen nach dem Stoß im Schwerpunktsystem

$\vec{c} \quad$ Geschwindigkeit des Schwerpunktes

Dabei seien die Massen der Teilchen $m_{1,2}$ vor dem Stoß und $m_{1,2}^{\prime}$ nach dem Stoß. Bekannt sind als Eingangsgrößen die Laborgeschwindigkeiten vor dem Stoß sowie aus der Messung doppelt differentieller Querschnitte die Intensitätsverteilung $I\left(\Theta_{L A B}, \vec{v}_{1}^{\prime}\right)$. Die Eingangsgrößen im Schwerpunktsystem lassen sich direkt berechnen. Die Geschwindigkeit des Schwerpunktes ergibt sich zu

$$
\vec{c}=\frac{m_{1}}{m_{1}+m_{2}} \vec{v}_{1}+\frac{m_{2}}{m_{1}+m_{2}} \vec{v}_{2}
$$

daraus folgen dann die Geschwindigkeiten $\vec{u}_{1}$ und $\vec{u}_{2}$. Da $\vec{u}_{1}$ und $\vec{u}_{2}$ kollinear sind, kann jetzt mit den Beträgen $u_{1}$ und $u_{2}$ weitergerechnet werden. Die kinetische Energie der Translation ist dann im Schwerpunktsystem

$$
E_{T R}=\frac{1}{2} m_{1} u_{1}^{2}+\frac{1}{2} m_{2} u_{2}^{2}
$$

Die Größen $\Theta_{C M}$ und $u_{1}$ ergeben sich jetzt direkt aus dem Newton-Diagramm. Aufgrund der Impulserhaltung kann $u_{2}$ berechnet werden, so daß auch bei Nachweis nur eines Teilchens die gesamte kinetische Energie nach dem Stoß festgestellt werden kann. Zur Bestimmung 
der Intensität im Schwerpunktsystem ist noch die Jacobi-Determinante der Transformation anzuwenden. Anschaulich betrachtet wird dadurch das feste Winkelelement $d \Omega$ des Detektors in die korrespondierende Größe $d \omega$ im Schwerpunktsystem umgerechnet. Im gegebenen Experiment ergibt sich die Jacobideterminante $\frac{v_{1}^{\prime 2}}{u_{1}^{\prime 2} \cos \zeta}$ für elastische Streuung (die Bedeutung des Winkels $\zeta$ ergibt sich aus Abb. 2.2) und $\frac{v_{1}^{\prime 2}}{u_{1}^{\prime 2}}$ für reaktive und inelastische Streuung, da keine einzelnen Quantenzustände aufgelöst werden [8].

Die kinematischen Eigenschaften eines Streusystems, abzulesen im Newton-Diagramm, können sehr verschieden sein; so gibt es Newton-Diagramme, für die zwei Schwerpunktswinkel auf demselben Laborwinkel nachgewiesen werden. Dieser Fall spielt in der vorliegenden Arbeit jedoch keine Rolle, so daß er bei der Diskussion von Newton-Diagrammen im folgenden nicht berücksichtigt wird.

Im Grenzfall der Streuung eines leichten, schnellen Teilchens an einem schweren, langsamen Teilchens ergibt sich eine Situation, in der das schwere Teilchen als fast stehend angesehen werden kann. Für den Nachweis des leichteren Teilchens, das hier immer als Primärteilchen angesehen wird, ergibt sich eine Situation entsprechend Abb. 2.2. Dieser Fall soll im folgenden als ,asymmetrisch“ klassifiziert werden. Die sich daraus ergebenden Auswirkungen werden in Kap. 4 diskutiert.

\subsection{Anregung von Natrium $3^{2} S_{1 / 2} \rightarrow 3^{2} P_{3 / 2}$}

Im Experiment wird die Streuung an $\mathrm{Na}\left(3^{2} P_{\frac{3}{2}}\right)$ - später einfach als $\mathrm{Na}(3 \mathrm{P})$ bezeichnet - untersucht. Daher sollen hier zunächst die für die Präparation dieses Zustandes notwendigen Grundlagen dargestellt werden. In Abschnitt 4.1 wird dann diskutiert, wie erfolgreich die Präparation im beschriebenen Experiment ist.

\section{Der Übergang $3^{2} S_{\frac{1}{2}} \rightarrow 3^{2} P_{\frac{3}{2}}$}

Die Wellenlänge des betrachteten Übergangs liegt bei 588.9963 nm [30]. Der Übergang zeigt Hyperfeinaufspaltung, d. h. eine Kopplung des Drehimpulses der Elektronen J mit dem des Kerns I, begründet in der Wechselwirkung des magnetischen Kernmoments mit dem Magnetfeld der Elektronen. Es ergibt sich ein Gesamtdrehimpuls

$$
\mathbf{F}=\mathbf{J}+\mathbf{I} \quad \text { mit dem Betrag } \quad|\mathbf{F}|=\sqrt{F(F+1)} \hbar .
$$

Der Wertebereich für $F$ ist $[\mathbf{J}+\mathbf{I}, \mathbf{J}+\mathbf{I}-1, \ldots, \mathbf{J}-\mathbf{I}]$. Da für Natrium $\mathbf{I} \frac{3}{2}$ beträgt, ergeben sich also für den Grundzustand $3^{2} S_{\frac{1}{2}}$ die Werte 1 und 2 für $\mathbf{F}$ und für den $3^{2} P_{\frac{3}{2}}$-Zustand $F=0,1,2,3$. Die Auswahlregel für optische Übergänge ist

$$
\Delta \mathbf{F}=0, \pm 1
$$

Die dementsprechend möglichen optischen Übergänge sind in Abb. 2.3 eingetragen. 
Liegt ein schwaches Magnetfeld $\mathbf{B}_{\mathbf{0}}$ an, welches die Kopplung zwischen $\mathbf{I}$ und $\mathbf{J}$ nicht aufhebt, ergeben sich $2 F+1$ Orientierungsmöglichkeiten von $\mathbf{F} \mathrm{zu} \mathbf{B}_{\mathbf{0}}$, beschrieben durch die Quantenzahl $m_{F} . m_{F}$ kann dann die Werte $-F,-F+1, \ldots, F$ annehmen. Im Einzelnen gelten die Auswahlregeln

$$
\begin{array}{ll}
\Delta m_{F}=0, \pm 1 & \text { für spontane Emission } \\
\Delta m_{F}=0 & \text { für Absorption und stimulierte Emission } \\
& \text { bei linear polarisiertem }(\pi \text { - }) \text { Licht } \\
\Delta m_{F}= \pm 1 & \text { für Absorption und stimulierte Emission } \\
& \text { bei zirkular polarisiertem }\left(\sigma^{+} / \sigma^{-}\right) \text {Licht }
\end{array}
$$

In Abwesenheit eines Magnetfeldes sind die $m_{F}$-Niveaus entartet, bestimmen aber das statistische Gewicht des jeweiligen Hyperfeinstrukturniveaus. Weiterhin ändert eine Anregung mit polarisiertem Licht die Besetzung der $m_{F}$-Niveaus (s. u.).

Die Besetzung der beiden Hyperfeinstrukturniveaus des Grundzustandes gehorcht der Boltzmann-Verteilung. Aufgrund der geringen Energiedifferenz wird die Besetzung nur vom Entartungsgrad bestimmt. Es befinden sich also $5 / 8$ der Atome in $F=1$ und $^{3} / 8$ im $F=0$.

\section{Zyklische Anregung im Atomstrahl}

Im Experiment soll sich innerhalb des Streuvolumens ein großer Anteil des Natriums - idealerweise $100 \%$ - im angeregten Zustand befinden. Prinzipiell können sich bei optischer Anregung im Zwei-Niveau-System im Mittel höchstens 50 \% im angeregten Zustand befinden.

Um derartige Verhältnisse erreichen zu können, muß während der gesamten Durchflugszeit der Atome durch das Streuvolumen ein zyklischer Anregungsprozess durchgeführt werden, denn innerhalb der spontanen Lebensdauer von 16,06 ns [78] legen die Atome nur ca. $50 \mu \mathrm{m}$ zurück. Zyklisch bedeutet hier, daß alle Atome, die nach Absorption eines Photons und der entweder durch induzierte oder spontane Emission vermittelten Rückkehr in den Grundzustand wieder der Anregung zur Verfügung stehen, d. h. in der Lage sind, ein Photon zu absorbieren.

Zur kontinuierlichen Anregung taugliche Dauerstrich-Farbstofflaser weisen eine Linienbreite von $1 \mathrm{MHz}$ bis $10 \mathrm{MHz}$ auf. Die Anregung ist also spezifisch bezüglich der Hyperfeinstrukturniveaus. Es muß dann derart aus einem der beiden Grundzustandsniveaus angeregt werden, daß die durch spontane Emission zurückfallenden Atome nur diesen Zustand erreichen können. Dafür existieren die folgenden Möglichkeiten :

(a) $F=1 \rightarrow F^{\prime}=0$

(b) $F=2 \rightarrow F^{\prime}=3$

Möglichkeit (b) ist günstiger, da $5 / 8$ der Atome zur Verfügung stehen und die Frequenzdifferenz zum nächstliegenden Hyperfeinstrukturniveau des angeregten Zustands größer ist.

Um eine hinreichend große Besetzung im oberen Zustand zu erreichen, ist eine hohe Fluenz erforderlich. Dann wird jedoch über Sättigungsverbreiterung [12] auch der Übergang $F=2 \rightarrow F^{\prime}=2$ möglich. Aus diesem Zustand kann über spontane Emission der $F=1$ - Grundzustand erreicht werden, so daß die entsprechenden Atome dauerhaft dem Anregungsprozess verloren gehen (,trapping-effect"). Da die quantitative, rechnerische Behandlung sehr aufwendig ist [51], soll hier darauf verzichtet werden. Experimentelle Ergebnisse in Abschnitt 4.1 und in $[27,59,80]$ belegen jedoch, daß der Effekt für die erreichbare 
Anregungseffizienz eine wichtige Rolle spielt.

Um das Auftreten des trapping-effects zu vermeiden, kann

1. zirkular polarisiertes Licht verwendet werden. So stellt sich etwa bei links-zirkular polarisiertem Licht eine stationäre $m_{F}$-Verteilung ein, bei der der Anregungsprozess nur noch zwischen den Niveaus $F=2, m_{F}=2$ und $F^{\prime}=3, m_{F}=3$ erfolgt. Ein Übergang in $F^{\prime}=2$ ist aufgrund der Auswahlregel für $m_{F}$ verboten. Allerdings dauert es relativ lange bis zur Einstellung der stationären Verteilung [51]; während dieser Zeit können durchaus erhebliche Verluste nach $F=1$ entstehen.

2. Eine weitere Möglichkeit ist der Einsatz eines Zwei-Farben-Lasers, der die Übergänge $F=2 \rightarrow F^{\prime}=3$ und $F=1 \rightarrow F^{\prime}=2$ gleichzeitig pumpt. Da aus beiden Grundzustandsniveaus angeregt wird, können durch spontane Emission keine Verluste mehr herbeigeführt werden. Außerdem stehen statt $5 / 8$ alle Atome zur Anregung zur Verfügung. Dieser Weg der Zwei-Farben-Anregung ist für das dargestellte Experiment eingeschlagen worden.

\subsection{Düsenstrahlen}

Eine Standardmethode der Erzeugung von Atom- bzw. Molekularstrahlen ist die adiabatische Expansion eines Gases durch eine Düse ins Vakuum. Die Düse ist im einfachsten Fall ein Loch in einer dünnen Wand, d. h. die Dicke der Wand ist klein gegenüber dem Düsendurchmesser $D$. Da die Molekularstrahltechnik mittlerweile gut bekannt ist, sei auf die Literatur verwiesen $[54,61]$. Im folgenden wird nur eine kurze Einführung in Grundlagen gegeben.

Die Expansionsbedingungen werden durch die Knudsenzahl $K$ charakterisiert

$$
K=\frac{\lambda_{0}}{D}
$$

charakterisiert. $\lambda_{0}$ ist die mittlere freie Weglänge im Gasreservoir hinter der Düse. Ist $D$ groß gemessen an $\lambda_{0}(K>10)$, finden während der Expansion keine Stöße statt. Die Geschwindigkeitsverteilung im Gasreservoir gilt dann auch nach der Expansion ins Vakuum. Man spricht von einer Knudsen- oder Effusivquelle. Ist jedoch $K \ll 1$, führen die Teilchen beim Passieren des Loches eine große Zahl an Stößen aus. Dabei wird die Energie der inneren Freiheitsgrade und der Translation in gerichteten Massenfluß umgesetzt. In diesem Falle wird der Molekularstrahl Düsenstrahl genannt. Dabei wird den einzelnen Freiheitsgraden in verschiedenem Maße Energie entzogen. Da die Teilchen nach Verlassen der Expansionszone keine Stöße mehr untereinander ausführen, bleibt das Ungleichgewicht zwischen den Freiheitsgraden erhalten. Es ist daher nicht mehr möglich, eine Temperatur für die Teilchen anzugeben; entsprechend der in den einzelnen Freiheitsgraden enthaltenen Energie lassen sich allerdings jeweils auf einen Freiheitsgrad bezogene Temperaturen $T_{v i b}, T_{\text {rot }}, T_{\text {trans }}$ angeben, die sich aus der Besetzung der jeweiligen Zustände ergibt. Es sind Translationstemperaturen von wenigen Kelvin erreichbar. Zumindest für die Vibration liegt dann nur noch die Grundzustandsbesetzung vor. Damit ist die adiabatische Expansion ein sehr effizientes Verfahren zur Abkühlung von Gasen. 
Zur Charakterisierung der Strömungsverhältnisse kann das sog. Geschwindigkeitsverhältnis (,Speed-ratio“)

$$
S=\frac{u}{\alpha}
$$

verwendet werden. $\alpha$ ist die thermische Restgeschwindigkeit, die sich aus der Temperatur $\mathrm{zu} \sqrt{\frac{2 k T}{m}}$ ergibt, $u$ die Strömungsgeschwindigkeit.

\section{Strahlgeschwindigkeit}

Die Geschwindigkeitsverteilung der Teilchen im Molekularstrahl läßt sich beschreiben durch die Verteilungsfunktion

$$
f_{n}(v) d v=N_{n} v^{n} e^{-\frac{(v-u)^{2}}{\alpha^{2}}} d v
$$

Dabei ist $n$ abhängig von der Art der Beobachtung. Für die Betrachtung des Teilchenflusses muß $n=3$ gesetzt werden, für die Betrachtung der Teilchendichte hingegen $n=2$. Durch Bildung des Erwartungswertes aus Gl. (2.9) ergibt sich für die wahrscheinlichste Geschwindigkeit $v_{w}$ im Strahl

$$
v_{w}=u \cdot\left(\frac{1}{2}+\sqrt{\frac{1}{4}+\frac{n}{2 S^{2}}}\right) .
$$

Ist $S$ sehr groß, folgt daraus näherungsweise $v_{w}=u$. Dann ergibt sich für die wahrscheinlichste Geschwindigkeit im Düsenstrahl näherungsweise

$$
v_{w} \approx u=\sqrt{2 \frac{\gamma}{\gamma-1} \frac{k T_{0}}{m}},
$$

wobei $T_{0}$ die Düsentemperatur und $\gamma$ der Adiabatenexponent des expandierten Gases ist.

\section{Expansion in Trägergas - ,Seeded Beams ${ }^{6}$}

Wie aus Gl. (2.11) hervorgeht, ist die Strahlgeschwindigkeit im wesentlichen von $\gamma$ und der Teilchenmasse $m$ des Gases abhängig, wenn die Düsentemperatur konstant gehalten wird. Für gleiches $\gamma$ ergibt sich nach Gl. (2.11) eine größere Strahlgeschwindigkeit für kleine $m$. Um die Strahlgeschwindigkeit zu verändern, kann nun das zu expandierende Gas A, dessen Teilchen die Masse $m$ haben, mit einem Trägergas B, dessen Teilchen die Masse $m_{t} \neq m$ haben, gemischt werden. Diese Technik, die allgemein "seeded-beam"-Technik genannt wird, dient in der Regel der Steigerung der Strahlgeschwindigkeit. Beträgt der Anteil des Gases mit der Masse A $x$, ergibt sich für die „Mischmasse“

$$
m_{s}=x m+(1-x) m_{t}
$$

Setzt man $m_{s}$ in Gl. (2.11) ein, ergibt sich die Strahlgeschwindigkeit des mit Trägergas expandierten Molekularstrahls. 
Wie gesagt, kann die Expansion in Trägergas dazu eingesetzt werden, die Strahlgeschwindigkeit gezielt zu verändern. Für Substanzen, deren Dampfdruck zur Erzeugung eines Düsenstrahls nicht ausreicht, kann ein ausreichender Expansionsdruck erreicht werden. Zur Beurteilung der Verhältnisse im dargestellten Experiment ist der Dampfdruck für Natrium im verwendeten Temperaturbereich $\left(400^{\circ}\right.$ bis $\left.600^{\circ}\right)$ in Abb. 2.4 angegeben. Für die verwendeten Trägergasdrücke von 500 mbar bis 2 bar ist also die Geschwindigkeit des Trägergases bestimmend für die Strahlgeschwindigkeit.

\subsection{Pseudostatistische Laufzeitanalyse}

Um die Energie der gestreuten Teilchen nach dem Stoß bestimmen zu können, wird eine Analyse der Teilchenlaufzeit zwischen Streuung und Detektor durchgeführt. Dabei wird im Idealfall zum Zeitpunkt der Streuung - eine an-aus-Modulation des Streusignals nach einer bestimmten Sequenz vorgenommen. Dazu wird ein Zerhacker - im folgenden Chopper genannt - verwendet. Die detektierten Teilchen werden zeitabhängig gezählt und durch Vergleich des Detektorsignals mit der Sequenz wird die Laufzeit bestimmt.

Dies soll im folgenden quantitativ dargestellt werden :

Das Detektorsignal setzt sich zusammen aus einem Anteil $S$, der auf Teilchen zurückgeht, die den Chopper durchlaufen haben, sowie einem Anteil $U$, der keine Modulationsinformation enthält (i. a. der Untergrund). Da $U$ keine Interaktion mit dem Chopper gehabt hat, ist $U$ keine Funktion der Zeit im Gegensatz zu $S=S(t)$. Der Chopper stellt eine endliche Anzahl $n$ von Zeitintervallen zur Verfügung, in denen Teilchen passieren können oder nicht; diese Abfolge gebe der Vektor $\vec{Q}$ an. Der elementare Zeitschritt des Choppers wird Kanalbreite $b_{K}$ genannt. Sinnvoll ist jetzt eine Betrachtung, in der die Zeit mit der Kanalbreite diskretisiert ist; $S(t)$ kann dann als Vektor $\vec{S}$ der Länge $n$ betrachtet werden. Die Detektorfunktion $\vec{D}$ wird ebenfalls in diskreten Intervallen der Kanalbreite aufgenommen. Für die Aufnahme stehen genausoviele Kanäle zur Verfügung, wie auf dem Chopper enthalten sind. Also hat $\vec{D}$ ebenfalls die Länge $n$.

Zum Startzeitpunkt t=0 werden der Ablauf der Sequenz $\vec{Q}$ und die Messung der Detektorfunktion $\vec{D}$ gestartet; die Sequenz $\vec{Q}$ wird dauernd wiederholt. Ist nun der k-te Kanal der Sequenz offen, so kommt im Detektor $\vec{S}$ um k Kanäle verschoben an. Die Detektorfunktion enthält also eine Summe aus verschobenen $\vec{S}$. Mathematisch aus gedrückt also ist

$$
\vec{D}=\mathbf{A} \vec{S}+U \vec{E}
$$

wobei die $n \times n$-Matrix A in folgendem Zusammenhang zu $\vec{Q}$ steht: 


$$
\mathbf{A}=\left(\begin{array}{c}
\vec{Q} \\
\vec{Q} \text { um 1 Kanal verschoben } \\
\ldots \\
\\
\ldots \\
\vec{Q} \text { um n Kanäle verschoben }
\end{array}\right)
$$

$\vec{Q}$ muß nun so gewählt sein, daß A invertierbar ist; eine solche Sequenz $\vec{Q}$ heißt pseudostatistisch. Dann erhält man durch Anwendung von $\mathbf{A}^{-1}$

$$
\vec{S}+\mathbf{A}^{-1} \vec{E} U=\mathbf{A}^{-1} \vec{D}
$$

Im gegebenen Experiment wird eine Sequenz $\vec{Q}$ eingesetzt, die aus 127 Kanälen besteht, von denen 64 durchlässig und 63 geschlossen sind. In diesem Fall ergibt sich (die Rechnung soll hier im einzelnen nicht nachvollzogen werden)

$$
\mathbf{A}^{-1} \vec{E}=\vec{E}
$$

im allgemeinen Fall

$$
\mathbf{A}^{-1} \vec{E}=k \vec{E} \text { mit } k \varepsilon \mathbb{Z}
$$

Damit ergibt sich aus 2.14

$$
\vec{S}+U=\mathbf{A}^{-1} \vec{D} .
$$

Damit ergibt sich dasjenige Resultat, das bei einem Experiment mit einem Einzelschlitzchopper mit einem Schlitz der Breite $b_{k}$ und einem Aufnahmesystem der Kanalbreite $b_{k}$ erzielt worden wäre. Daher kann im folgenden die Laufzeitmessung betrachtet werden, als sei sie mit einem entsprechenden Einzelschlitzchopper durchgeführt worden. Der Vorteil der pseudostatistischen Methode liegt darin, daß etwas über 50\% der Teilchen durchgelassen werden, während die Einzelschlitzmethode nur einen kleinen, von der gewünschten Auflösung abhängigen Anteil (im gegebenen Fall $\frac{1}{127} \approx 0.8 \%$ ) durchläßt. 


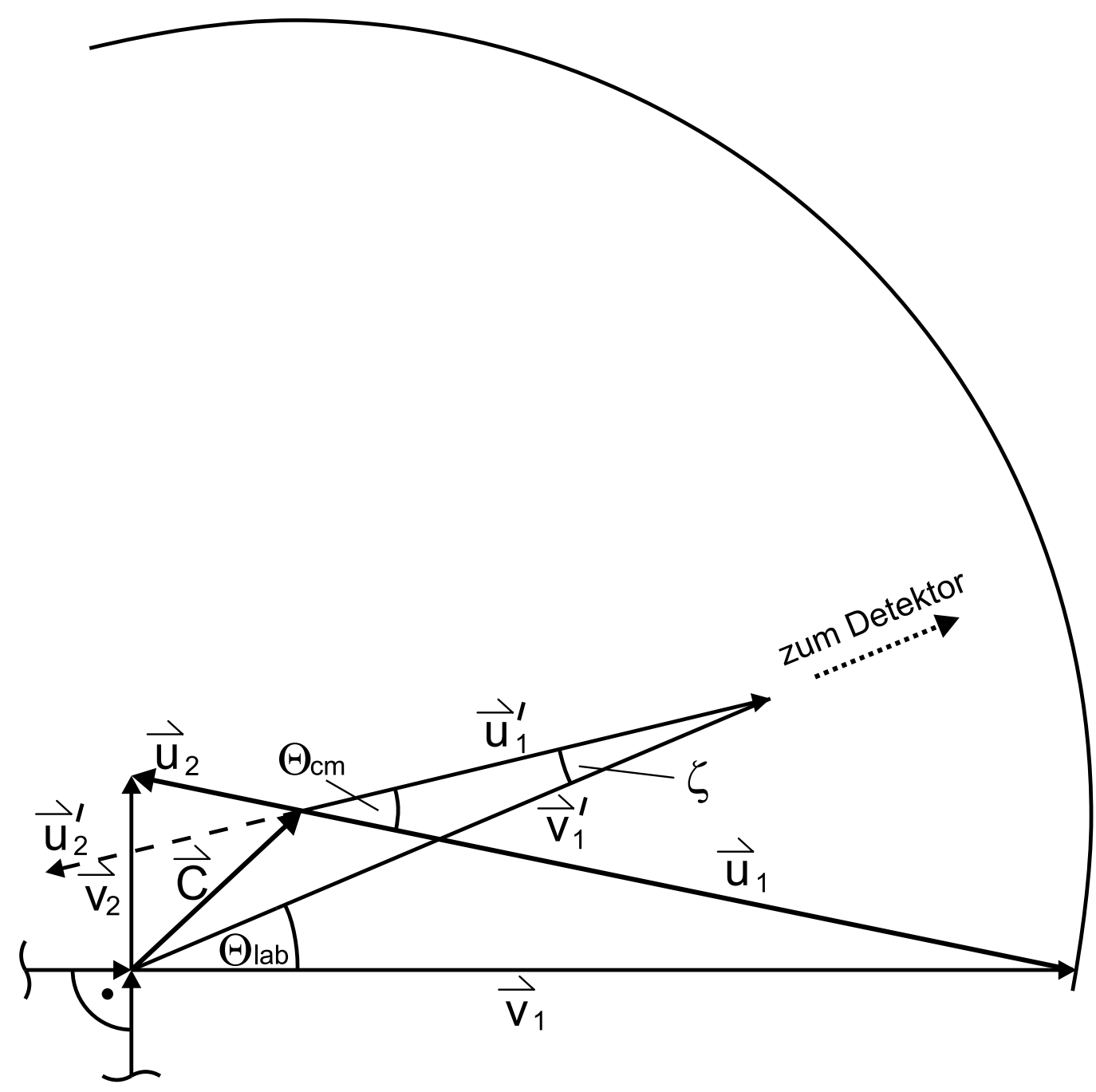

Abb. 2.2: Newton-Diagramm für den Fall inelastischer Streuung. Die Bedeutung der Symbole ergibt sich aus dem Text. 


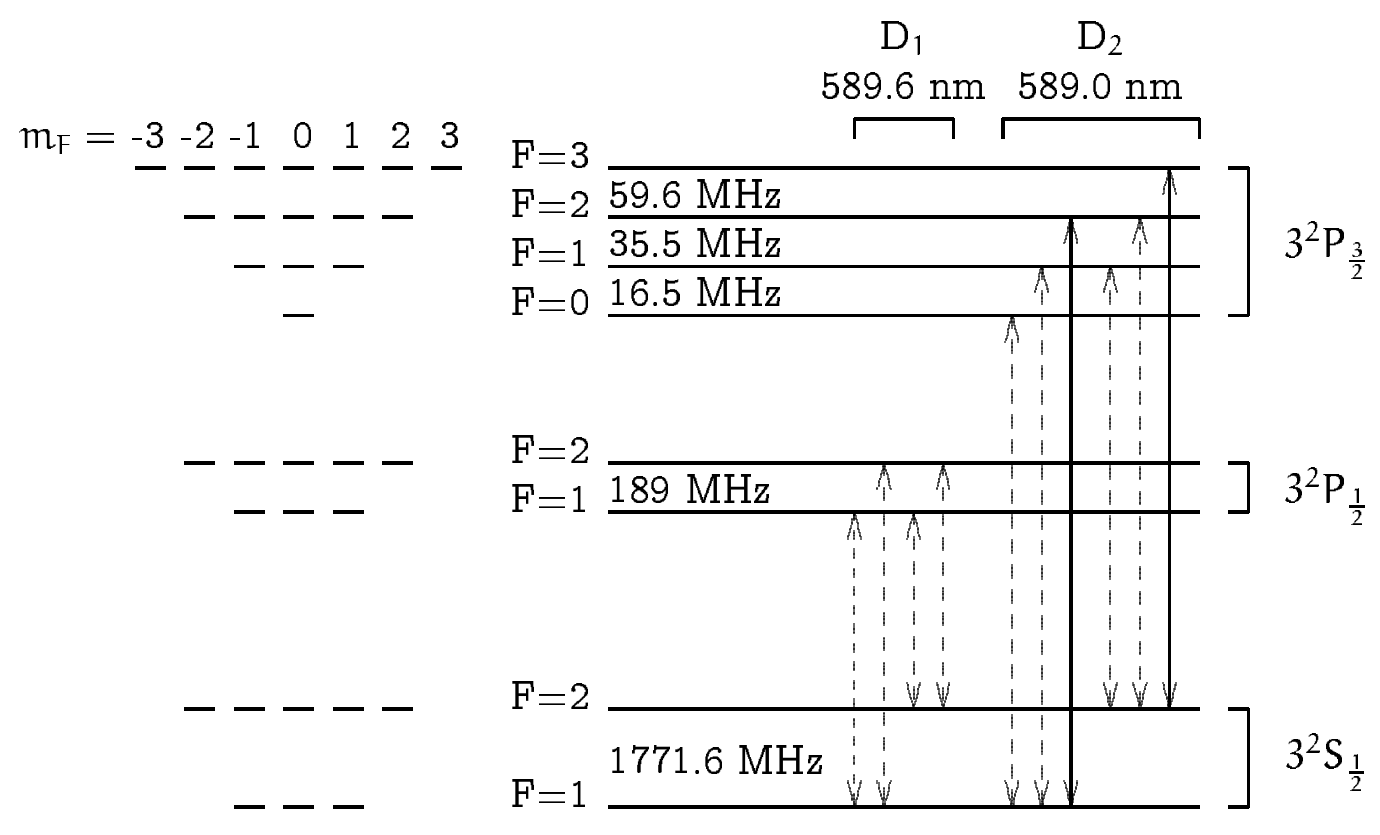

Abb. 2.3: Hyperfeinstrukturaufspaltung der Natrium-Übergänge $3^{2} S_{\frac{1}{2}} \rightarrow 3^{2} P_{\frac{1}{2}}$ und $3^{2} S_{\frac{1}{2}} \rightarrow 3^{2} P_{\frac{3}{2}}$. Die entsprechend der Auswahlregel 2.6 erlaubten Übergänge sind eingezeichnet, die verwendeten als durchgezogene Linien. Rechts sind die entarteten $m_{F}$-Niveaus angegeben.

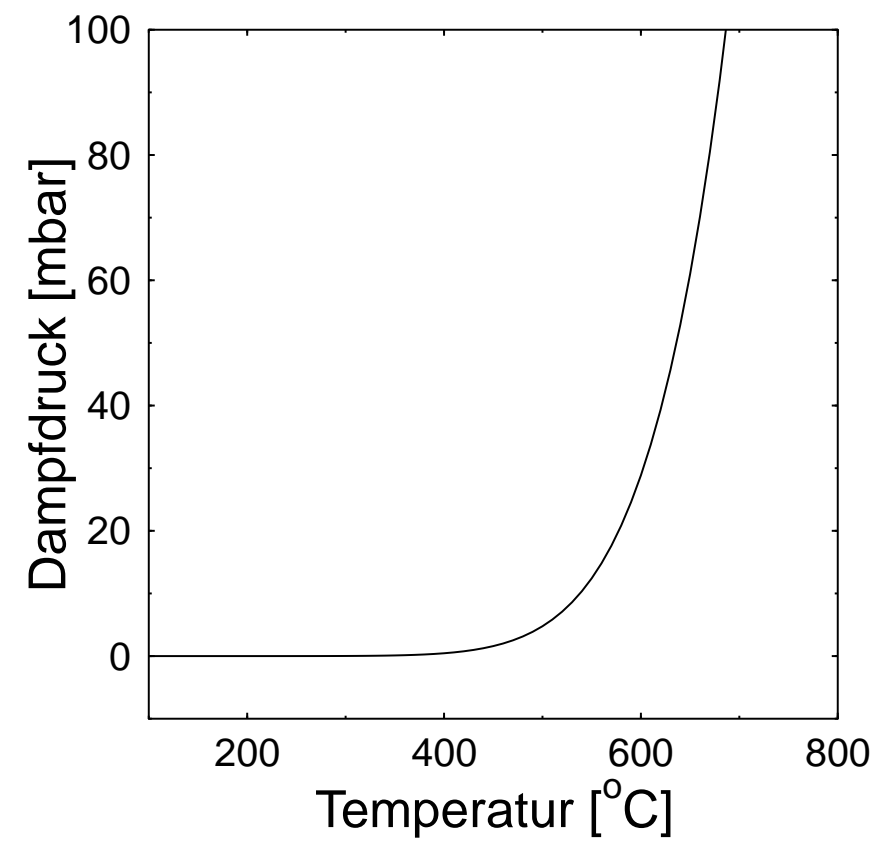

Abb. 2.4: Dampfdruckkurve von Natrium, entnommen aus [70] 


\section{Kapitel 3}

\section{Experimentelle Realisation}

\subsection{Vakuumapparatur}

Abb. 3.1 stellt die Aufteilung der Vakuumapparatur dar. Im folgenden ist eine Beschreibung der Komponenten gegeben :

Die Primärkammer dient der Aufnahme des Natrium-Ofens; mit der folgenden differentiellen Pumpstufe ist sie durch einen geheizten Skimmer (Durchmesser $600 \mu \mathrm{m}$ ) verbunden. Das Hochvakuum wird mit einer Diffusionspumpe Typ Leybold DI 6000 $\left(6000 \frac{1}{\mathrm{~s}}\right)$ erzeugt. Zum Ausfrieren des Natriums befindet sich ein flüssig-StickstoffBehälter in der Kammer, an den diverse Kupferbleche so angebracht sind, daß das austretende Natrium quantitativ ausgefroren werden kann. Dennoch sammelt sich eine gewisse Menge Natrium bzw. dessen Reaktionsprodukte im Öl der Diffusionspumpe; die Pumpleistung wird dadurch offenbar nicht negativ beeinflußt. Als Vorpumpsystem kommt eine Kombination aus einer zweistufigen Drehschieberpumpe (60 $\frac{\mathrm{m}^{3}}{\mathrm{~h}}$ ) und einer Boosterpumpe zum Einsatz. Ohne Belastung wird in der Primärkammer ein Druck vom $5 \cdot 10^{-6}$ mbar erreicht; beim höchsten verwendeten Seedegasdruck von 2 bar liegt der Kammerdruck bei $5 \cdot 10^{-4}$ mbar.

Die differentielle Pumpstufe ist mit einer Diffusionspumpe (1000 $\left.\frac{1}{\mathrm{~s}}\right)$ ausgestattet und an das Vorvakuumsystem der Primärkammer angeschlossen. An der Verbindungsstelle zur Hauptkammer befindet sich ein Schiebeventil.

Die Streukammer verfügt selbst über keine Pumpe. Über die seitlich angeflanschten Kammern, die vollständig zur Streukammer hin geöffnet sind, wird dennoch eine annehmbare Pumpleistung erzielt. Weiterhin befinden sich zwei mit flüssigen Stickstoff gefüllte Behälter in der Streukammer: Einer direkt unterhalb des Streuzentrums, ein weiterer im Kammerdeckel. Von diesem aus werden die Ummantelungen der Detektoren mit flüssigen Stickstoff versorgt; an einen weiteren Kreislauf angeschlossen sind das gekühlte Flugrohr sowie ein Kühlblech zum Abfangen von Natrium. An diesen Bauteilen werden Temperaturen von $-140^{\circ} \mathrm{C}$ bzw. $-120^{\circ} \mathrm{C}$ erreicht. Da der Sekundärstrahl direkt in die Streukammer eintritt und weiterhin Drücke realisiert werden müssen, die Einzelstoßbedingungen garantieren, ist die Pumpleistung nur bei Verwendung gut 


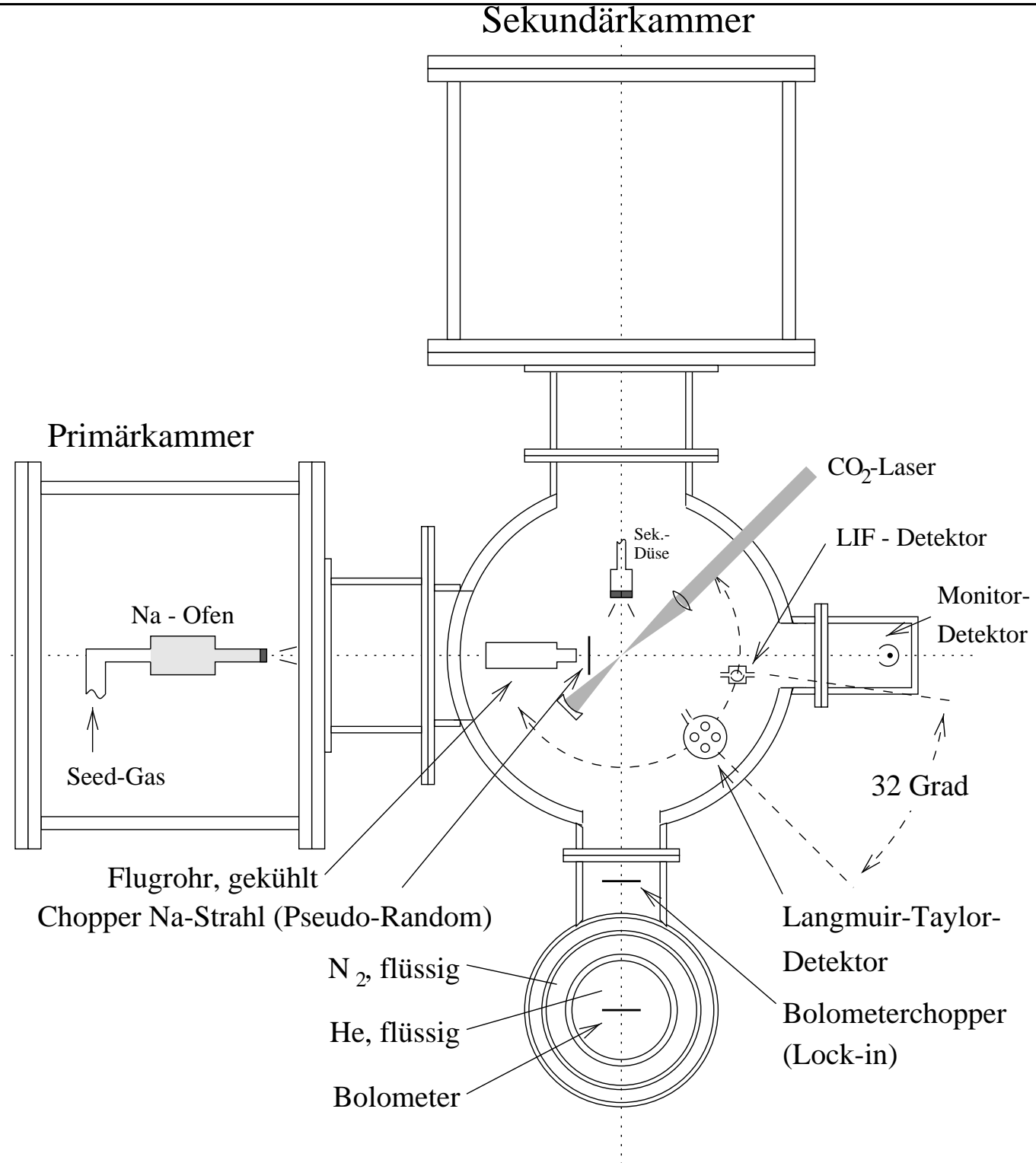

Abb. 3.1: Schnitt durch die Apparatur in Strahlebene. Die beiden Detektoren sind am drehbaren Deckel der Streukammer aufgehängt. Die erreichbaren Winkel sind ca. $-30^{\circ}$ bis $108^{\circ}$ für den LIF- und ca. $0^{\circ}$ bis $140^{\circ}$ für den LT-Detektor (bei einigen Messungen nur bis $86^{\circ}$ bzw. $\left.100^{\circ}\right)$

auszufrierender Gase oder sehr geringem Einlaßdruck für den Sekundärstrahl ausreichend. Im unbelasteten Zustand wird - mit Stickstoffüllung - ein Enddruck von $2 \cdot 10^{-7}$ mbar erreicht. Der zulässige Betriebsdruck bei Einsatz des Sekundärstrahls ist gasartabhängig (s. Abschnitt 2.5).

An die Streukammer angeflanscht sind drei weitere Kammern, die mit je einer Diffusionspumpe $\left(6000 \frac{1}{\mathrm{~s}}, 3000 \frac{1}{\mathrm{~s}}\right.$ und $\left.1000 \frac{\mathrm{l}}{\mathrm{s}}\right)$ bestückt sind. Jede ist mit einer wassergekühlten Dampfsperre versehen, um Kontamination der Streukammer mit Pumpenöl zu vermei- 


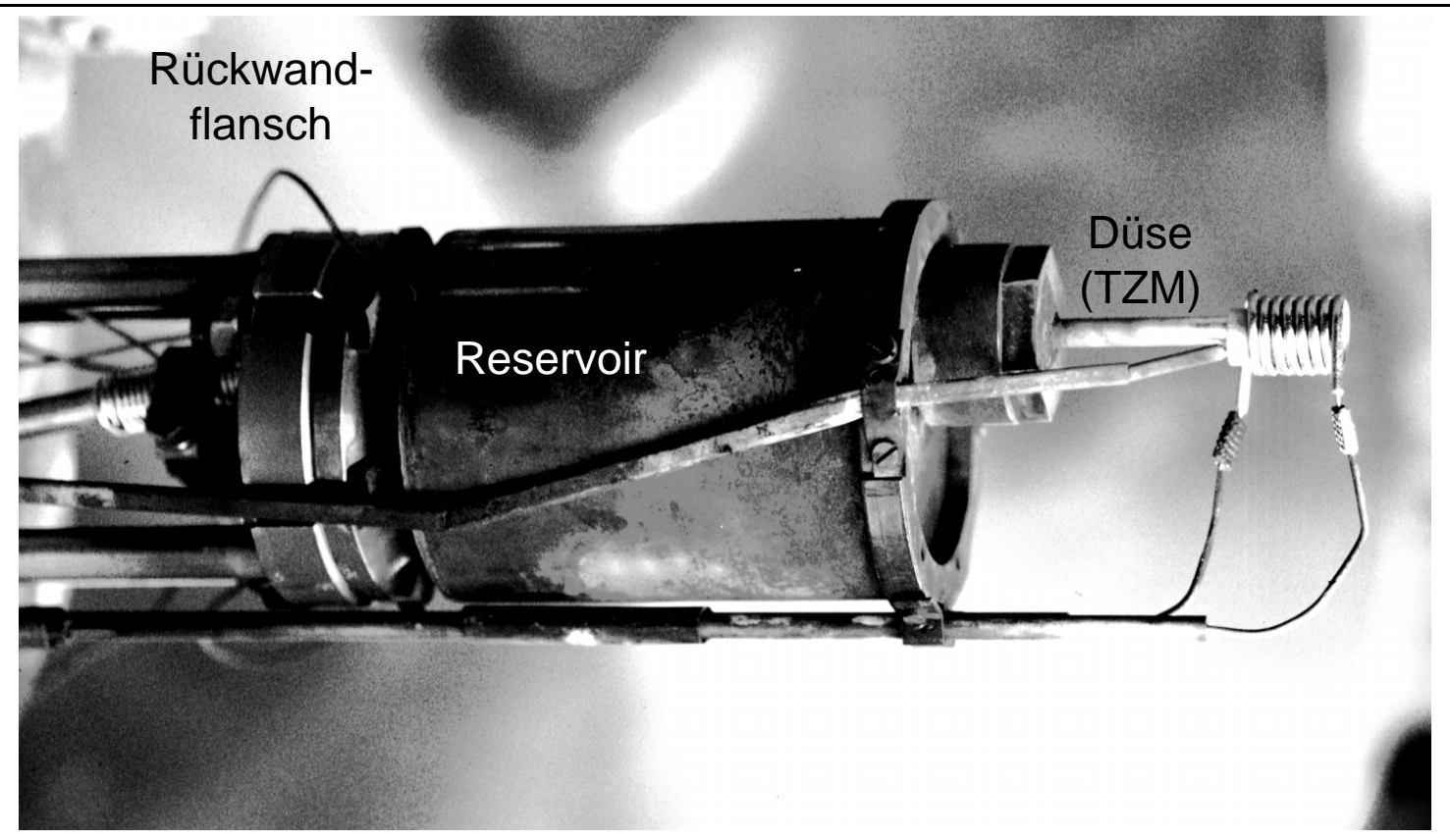

Abb. 3.2: Na-Ofen.

den. Alle Diffusionspumpen sind an ein gemeinsames, leistungsfähig dimensioniertes Vorpumpensystem, bestehend aus einer Drehschieberpumpe $\left(25 \frac{\mathrm{m}^{3}}{\mathrm{~h}}\right)$ und einer Wälzkolbenpumpe $\left(1000 \frac{\mathrm{m}^{3}}{\mathrm{~h}}\right)$, angeschlossen.

\subsection{Molekularstrahlquellen}

\section{Primärstrahl (Natrium-Atomstrahl)}

\section{Aufbau}

Zur Erzeugung des Primärstrahls wird eine "seeded-beam"Quelle [61, 39] (Abb. 3.2, im folgenden Ofen genannt) verwendet : Im geheizten zylindrischen Reservoir befindet sich das Natrium, über ein Einlaßrohr tritt das Trägergas - hier $\mathrm{H}_{2}$, He oder $\mathrm{Ne}$ - ein. Dem Einlaßrohr gegenüber ist eine Röhre angebracht, deren Ende als Düse mit einer Öffnung von $120 \mu \mathrm{m}$ ausgebildet ist. Dieses Bauteil, im folgenden als Düse bezeichnet, wird mittels einer Heizwicklung aus hochtemperaturbeständigem Heizleiter [4] auf bis zu 1400 ${ }^{\circ} \mathrm{C}$ aufgeheizt (Abb. 3.3 zeigt die Anbringung des Heizleiters). Da das Ofenmaterial Inconell dieser Temperatur nicht

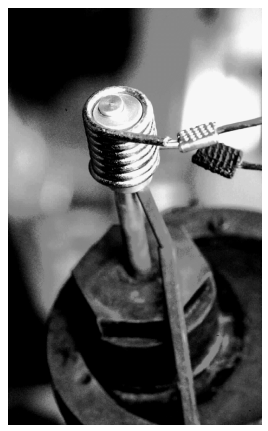

Abb. 3.3: Düse standhalten würde, wurde für die Düse Wolfram verwendet; dabei hat sich als nachteilig erwiesen, daß durch die Materialstruktur des Wolframs die Düse im Betrieb zur Aufweitung neigt. Durch die Verwendung von TZM, einer Legierung aus 99.4 \% Molybdän, $0.5 \%$ Tantal 
und $0.1 \%$ Zirkon wurde die Lebensdauer der Düsen erheblich gesteigert. TZM hat weiterhin den Vorteil, daß es im Gegensatz zu Wolfram spanender Bearbeitung zugänglich ist und die Düsen mithin einfacher zu fertigen sind. Die Düse wird mittels einer Konusdichtung und einer Überwurfmutter, die unter Erwärmung angezogen wird, am Ofen befestigt.

Die Rückwand des Reservoirs, die auch das Gaseinlaßrohr trägt, kann zum Füllen des Ofens abgenommen werden. Die Dichtung der Rückwand gegen den Ofen wird mit einem Conflat-ähnlichen Mechanismus erreicht; zwischen zwei Schneidkanten wird ein Kupferring eingedrückt. Um Dichtigkeit auch bei Betriebstemperatur zu erreichen, wird ein verhältnismäßig dicker Kupferring verwendet und dieser mit Hilfe von besonders reißfesten Schrauben (M6, $800 \mathrm{~N} / \mathrm{mm}^{2}$ ) besonders tief eingeschnitten.

Das Reservoir und - zur Vermeidung von Kondensation - das Gaseinlaßrohr tragen je eine Heizwicklung aus konventionellem Heizleiter (Philips Thermocoax). Die Stromversorgung wird mit hochwertigen Niederspannungsnetzteilen durchgeführt. Eine Temperaturstabilisierung hat sich nicht als notwendig erwiesen.

\section{Praktischer Betrieb}

In den Ofen wird soviel Natrium eingefüllt, daß dieser später (nach dem Aufschmelzen) etwa halb gefüllt ist. Dabei wird die Schutzflüssigkeit (Petroleum bzw. Parafinum subliquidum) so weit wie möglich entfernt. Um die verbliebene Schutzflüssigkeit zu entfernen, wird der Ofen nach dem Einbau in die Vakuumkammer mehrere Stunden bei ca. $120^{\circ} \mathrm{C}$, ausgeheizt"; dabei wird er durch den Einlaß für Trägergas evakuiert. Mit einer so erzeugten Füllung ist über eine Woche Dauerbetrieb möglich.

Danach kann das Trägergas in den Ofen eingelassen und auf Betriebstemperatur (s.u.) aufgeheizt werden. Es hat sich als wichtig erwiesen, das Trägergas durch eine $\mathrm{N}_{2}$-Kühlfalle zu leiten, da sonst Verunreinigungen - vermutlich Pumpenöl - am Gaseinlaß mit dem Natrium reagieren und die entstehende Substanz den Gaseinlaß zusetzt. Wie erwähnt kann durch Variation von Düsentemperatur, Reservoirtemperatur sowie Druck und Art des Trägergases die Geschwindigkeit des Natriumstrahl variiert werden; in Tabelle 3.1 werden typische Betriebsbedingungen angegeben.

\begin{tabular}{|r||l|l||l|l|}
\hline \multicolumn{1}{|c||}{} & \multicolumn{2}{c||}{$\mathrm{v}_{\mathrm{w}}=2280 \frac{\mathrm{m}}{\mathrm{s}}$} & \multicolumn{2}{c|}{$\mathrm{V}_{\mathrm{w}}=4800 \frac{\mathrm{m}}{\mathrm{s}}$} \\
\hline Trägergas & \multicolumn{2}{c|}{$\mathrm{He}$} & \multicolumn{2}{c|}{$\mathrm{H}_{2}$} \\
\hline & el. Heizleistung & Temperatur & el. Heizleistung & Temperatur \\
\hline Düse & $210 \mathrm{~W}$ & $915^{\circ} \mathrm{C}$ & $365 \mathrm{~W}$ & $1130^{\circ} \mathrm{C}$ \\
Reservoir & $150 \mathrm{~W}$ & $520^{\circ} \mathrm{C}$ & $150 \mathrm{~W}$ & $549^{\circ} \mathrm{C}$ \\
\hline
\end{tabular}

Tab. 3.1: Betriebsbedingungen des Ofens für 2 verschiedene Primärstrahlgeschwindigkeiten. Insgesamt kann ein Bereich von etwa $1500 \frac{\mathrm{m}}{\mathrm{s}}$ bis $5000 \frac{\mathrm{m}}{\mathrm{s}}$ abgedeckt werden. Im untersten Bereich ist allerdings die Strahlqualität hinsichtlich Breite der Geschwindigkeitsverteilung und Intensität von nur mäßiger Qualität. 


\section{Sekundärstrahl}

Als Sekundärdüse wird eine $10 \mu \mathrm{m}$-Lochdüse verwendet. Der geringe Düsendurchmesser ist darin begründet, daß die Expansion direkt in die Streukammer erfolgt und somit der mögliche Gasanfall durch die zur Verfügung stehende Pumpleistung begrenzt ist. Der beheizbare Düsenträger ist auf einer von außerhalb der Apparatur bedienbaren x-y-z-Verstellung montiert.

Da die Bestimmung der Geschwindigkeit des Sekundärstrahls nicht möglich ist, muß für diesen Wert auf Messungen aus anderen Experimenten zurückgegriffen werden oder sie muß berechnet werden. Dies ist bei der Darstellung der einzelnen Experimente angegeben.

\subsection{Lasersystem}

\section{Der Farbstoff-Laser}

Zur Erzeugung des Lichtes für die Natrium-Anregung wird ein kommerzieller DauerstrichFarbstoff-Laser (Coherent CR 599-21) verwendet. Es handelt sich um einen linear aufgebauten Laser mit gefaltetem Resonator und freiem Farbstoff-Strahl. Als Farbstoff wird Rhodamin 6G eingesetzt; die benötigte Wellenlänge von $589 \mathrm{~nm}$ liegt in der Nähe des Fluoreszenzmaximums dieses Farbstoffes. Der Laser-Resonator enthält zur Grobabstimmung der Wellenlänge ein Lyot-Filter. Einzel-Frequenz-Betrieb wird mit einer in den Resonator eingesetzten Einheit aus zwei Etalons erreicht; in dieser Einheit befindet sich weiterhin eine elektrisch drehbare Glasplatte (galvo-plate), die das Verändern der optischen Weglänge im Resonator und somit das Durchstimmen des Lasers ermöglicht. Zum Lasersystem gehört ein externes, temperaturstabilisiertes Etalon, auf dessen Transmissionswellenlänge der FarbstoffLaser elektronisch stabilisiert wird.

Um ausreichende Leistung und Stabilität zu erreichen, wurde der Farbstoff-Kreislauf durch ein gegenüber der Standardausstattung des Lasers erheblich leistungsfähigeres System ersetzt. Mittels einer speziellen Düse [1], die weniger Turbulenzen im Jet erzeugt, sowie einer stärkeren Farbstoff-Pumpe in Verbindung mit einem Gaspolster konnte der FarbstoffDurchsatz deutlich gesteigert werden. Diese Maßnahme war insofern notwendig, als mit dem bisherigen Pumpe-Düse-System auch im Bereich maximalen Durchsatzes die vom FarbstoffLaser erbrachte Leistung stark mit dem Durchsatz an Farbstofflösung variiert. Mit dem neuen System hingegen läßt sich Sättigung erreichen. Insgesamt wird im Einzel-Frequenz-Betrieb eine Leistung von $130 \mathrm{~mW}$ erreicht. Dabei kann die Laserfrequenz um maximal $30 \mathrm{GHz}$ durchgestimmt werden; das ist für die Bestimmung der Primärstrahlgeschwindigkeit (s. 2.4) in jedem Falle ausreichend. Laut Spezifikation liegt die Linienbreite unter $10 \mathrm{MHz}$; eigene Messungen mit einem durchstimmbaren Etalon (Burleigh, FSR $150 \mathrm{MHz}$ ) bestätigen dies. Die Langzeitstabilität ist durch das temperaturstabilisierte Referenz-Etalon bestimmt und mit $100 \mathrm{MHz} / \mathrm{h}$ spezifiziert. Praktisch ist die Langzeitstabilität zwar höher; allerdings wird die aufwendige Elektronik durch äußere Einflüsse leicht gestört. Dadurch werden Modensprünge des Lasers hervorgerufen, so daß eine häufige Kontrolle während der Messungen unabdingbar ist.

Als Pumplaser dient ein $\mathrm{Ar}^{+}$-Laser (Coherent Innova 90), der im multiline-Betrieb maximal $6 \mathrm{~W}$ abgibt. Der Farbstoff-Laser wird mit einer Pumpleistung von max. $4 \mathrm{~W}$ multiline 
betrieben.

\section{Der elektrooptische Modulator}

Um die Anregung des Natriums auf beiden Hyperfeinstrukturübergängen simultan zu ermöglichen, werden außerhalb des Laserresonators die beiden benötigten Frequenzen mittels eines elektrooptischen Modulator (EOM) aus dem Laserlicht erzeugt. Grundsätzlich sind noch andere Möglichkeiten denk- und verwendbar. In [76] ist ein linear aufgebauter Farbstofflaser beschrieben, dessen Auskoppelfenster durch ein Fabry-Perot-Etalon ersetzt wurde. Auf diese Weise produziert der Laser direkt die beiden benötigten Wellenlängen; allerdings ist der Aufwand für die gleichzeitige Stabilisierung des Lasers und des Etalons recht hoch, wird jedoch durch eine hohe Effizienz belohnt. Weiterhin wäre denkbar, zwei separate Lasersysteme zu verwenden; diese Möglichkeit führt allerdings zu einer dramatischen Steigerung der Anschaffungs- und Betriebskosten.

Elektrooptische Modulatoren werden für die effiziente Anregung bzw. Spinpolarisation von Natrium inzwischen von mehreren Gruppen [43, 59, 27, 5] eingesetzt. Da es sich jedoch nicht um ein kommerziell erhältliches Gerät handelt, soll die Funktionsweise hier dargestellt werden. Der Einsatz des Modulators an der verwendeten Apparatur und die damit verbundenen Konsequenzen sind ausführlich in [80] beschrieben.

\section{Grundprinzip und Funktionsweise}

Grundelement des EOM ist ein doppelbrechender Kristall (in diesem Fall aus $\mathrm{LiTaO}_{3}$ ). Durch diesen tritt das Laserlicht senkrecht zur optischen Achse hindurch. Die Polarisation des Lichts ist parallel zur optischen Achse, so daß das Licht den Weg des außerordentlichen Stahls nimmt. In Richtung der optischen Achse wird ein elektrisches Feld der Frequenz $\omega_{0}$ eingekoppelt. Damit wird - vermittelt vom linearen elektrooptischen Effekt - der Brechungsindex periodisch variiert. Ergebnis ist eine Phasenmodulation des durchtretenden Laserlichts. Auf eine formelmäßige Darstellung der Zusammenhänge soll hier verzichtet werden; sie finden sich in diversen Arbeiten zum Thema [82, 59, 80].

Als Ergebnis der entsprechenden Berechnungen erhält man das Frequenzspektrum des Lichts, nachdem es den Modulator passiert hat, in Abhängigkeit von den Kenngrößen des Kristalls, der Wellenlänge und dem elektrischen Wechselfeld. Dieses Frequenzspektrum enthält ausschließlich Anteile der Form $\omega_{\text {out }}=\omega_{\text {in }} \pm n \cdot \omega_{0}\left(\omega_{\text {in }}\right.$ und $\omega_{\text {out }}$ : Lichtfrequenz an der Eingangs- bzw. Ausgangsseite des Modulators, n : ganze Zahl). Man bezeichnet diese Frequenzanteile als Seitenbänder. Die Intensität des n-ten Seitenbandes ist gegeben durch das Quadrat der n-ten Besselfunktion des Modulationsindex $\eta$, einer für Kristall und Betriebsbedingungen charakteristischen Größe. Diese Intensität gilt gleichermaßen für das höherfrequente wie das niederfrequente Seitenband. Wie man Abb. 3.4 entnehmen kann, läßt sich für das erste Seitenband eine Intensität von $34 \%$ der Gesamtintensität erreichen. Mithin entsteht eine Situation, bei der zwei Bänder im Frequenzabstand $2 \omega_{0} 68 \%$ der eingestrahlten Leistung enthalten; damit ist die experimentelle Anforderung, zwei Laserlinen zu erhalten, prinzipiell erfüllt. Die eingekoppelte Mikrowellenfrequenz muß der halben Differenzfrequenz der gewünschten Laserlinien (s. Abschnitt 2.4), also $\frac{1712 \mathrm{MHz}}{2}=856 \mathrm{MHz}$ entsprechen. 


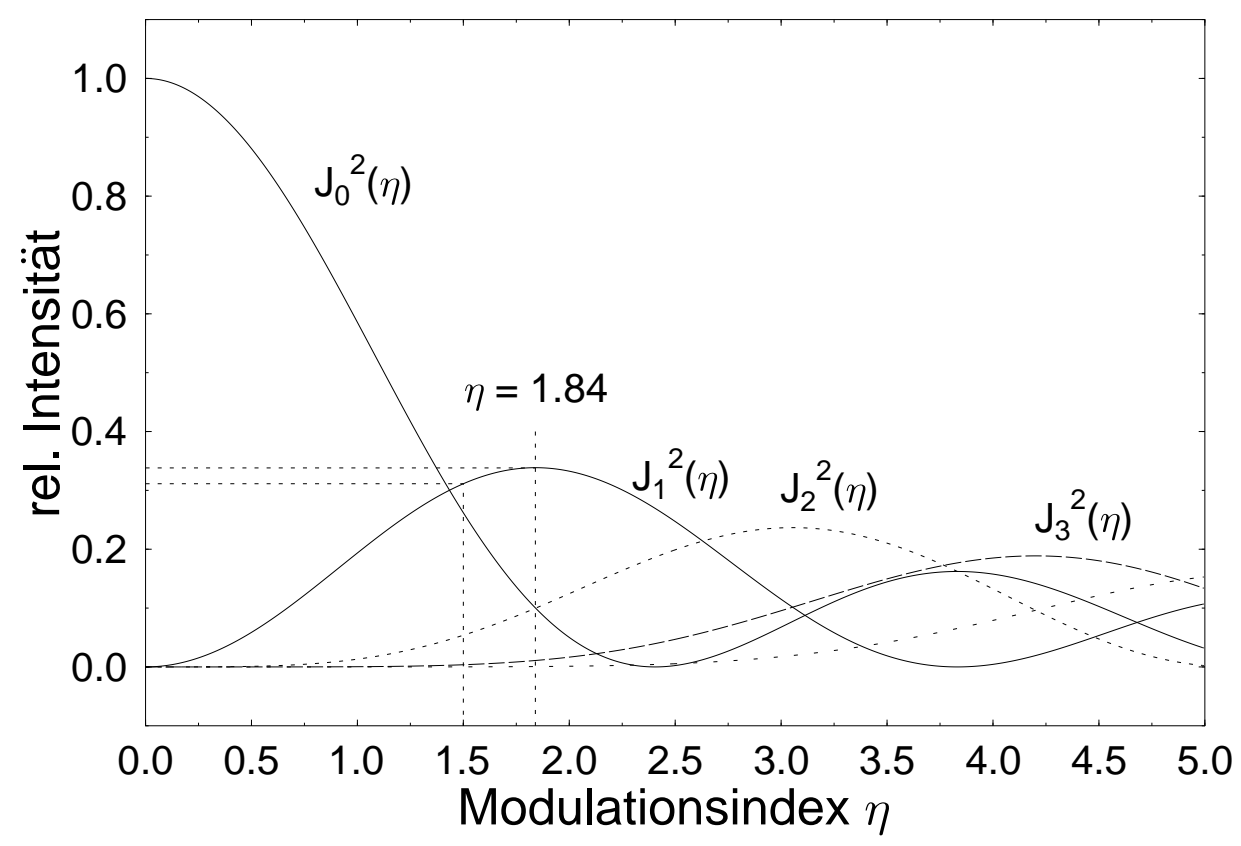

Abb. 3.4: Intensität der Seitenbänder in Abhängigkeit vom Modulationsindex $\eta$. Für die hier betrachtete Anwendung ist die Intensität der ersten Seitenbänder wesentlich. Man erkennt, daß diese in der Umgebung des optimalen Modulationsindex ein flaches Maximum aufweist; eine geringfügige Änderung des Modulationsindex führt nur zu einer Verschiebung der Intensität zwischen dem Träger und den zweiten Seitenbändern.

Allerdings muß noch der Modulationsindex von $\eta=1.84$ erreicht werden. Der Modulationsindex hängt folgendermaßen von den Bedingungen $a b$ :

$$
\eta=\frac{\pi n_{e}^{3} r_{3 z} U L}{\lambda d}
$$

mit

$n_{e}:$ Brechungsindex des außerordentlichen Strahls

$r_{3 z}:$ Komponente des el-opt. Tensors

$U$ : Amplitude des Wechselfeldes

$L, d, d$ : Länge, Breite und Höhe des Kristalls

$\lambda$ : eingestrahlte Wellenlänge

Durch die Auswahl des Kristallmaterials werden die Materialkonstanten $n_{e}$ und $r_{3 z}$ bestimmt. Für $\mathrm{LiTaO}_{3}$ sind $n_{e}=2.1878$ und $r_{3 z}=30.3 \cdot 10^{-12}$. Der Wert für $r_{3 z}$ ist im Vergleich 


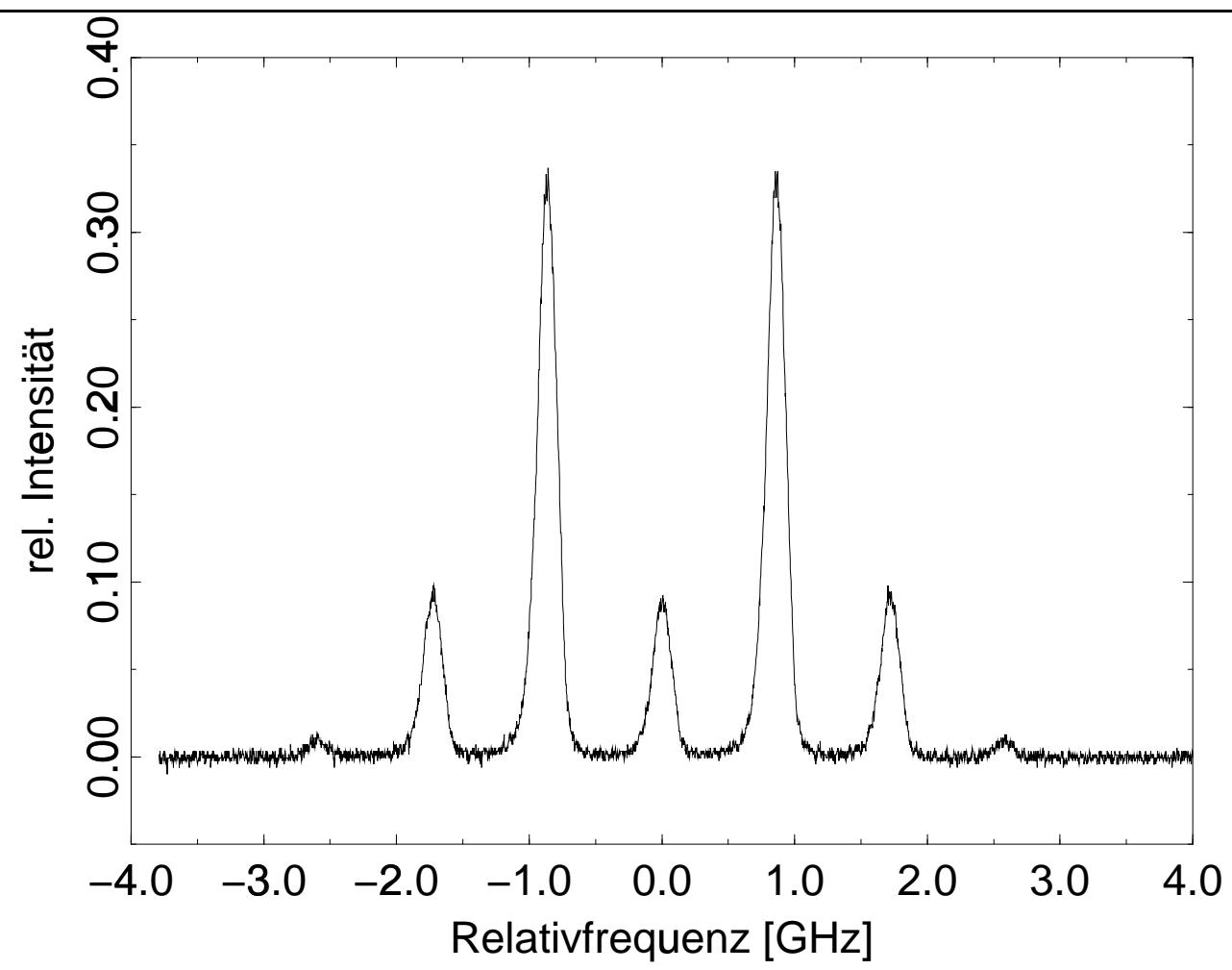

Abb. 3.5: Gemessenes Frequenzspektrum hinter dem EOM bei optimalen Betriebsbedingungen. Die spektrale Verteilung läßt auf einen Modulationsindex von 1.84 (s. Abb. 3.4) schließen. Das Spektrum wurde mit einem Interferometer mit einem FSR von $7.5 \mathrm{GHz}$ aufgenommen. Die relativ geringe Finesse dieses Interferometers ist für die Breite der Peaks verantwortlich.

mit anderen Materialien sehr hoch und führt daher zur Wahl von $\mathrm{LiTaO}_{3}$. Die Amplitude des Wechselfeldes muß nun noch so groß sein, daß bei den Abmessungen des Kristalls und der verwendeten Wellenlänge der geforderte Modulationsindex erreicht wird; das ist für $\mathrm{LiTaO}_{3}$ technisch möglich. Im verwendeten Aufbau ist der Kristall in einen Mikrowellenresonator eingesetzt, so daß der den Modulator versorgende HF-Generator eine Leistung von nur $1 \mathrm{~W}$ haben muß [5]. Damit verbunden ist allerdings, daß der Aufbau nur für einen engen Frequenzbereich genutzt werden kann.

\section{Der Modulator im Experiment}

Wie Abb. 3.5 zeigt, ist die Vorhersage, daß sich $68 \%$ der durchtretenden Leistung in zwei Linien im Abstand 1.772 GHz unterbringen lassen, experimentell realisierbar. Eine Abhängigkeit von der Leistung des Lasers wurde für die erreichbarem max. $150 \mathrm{~mW}$ nicht beobachtet. Eine Veränderung der Linienbreite konnte ebenfalls nicht festgestellt werden.

Sichergestellt werden muß noch, daß die beiden 1 . Seitenbänder die korrekten Frequenzen aufweisen. Es muß zum einen die Frequenz des Dye-Lasers auf die Mittenfrequenz der beiden Übergänge eingestellt werden. Zum anderen muß die Frequenz des HF-Generators auf die halbe Differenzfrequenz gebracht werden; dies ist einfach möglich, indem die Frequenz mit einem Zähler (Hameg HM 8022) kontrolliert wird. Die Einstellung ist dabei mit 


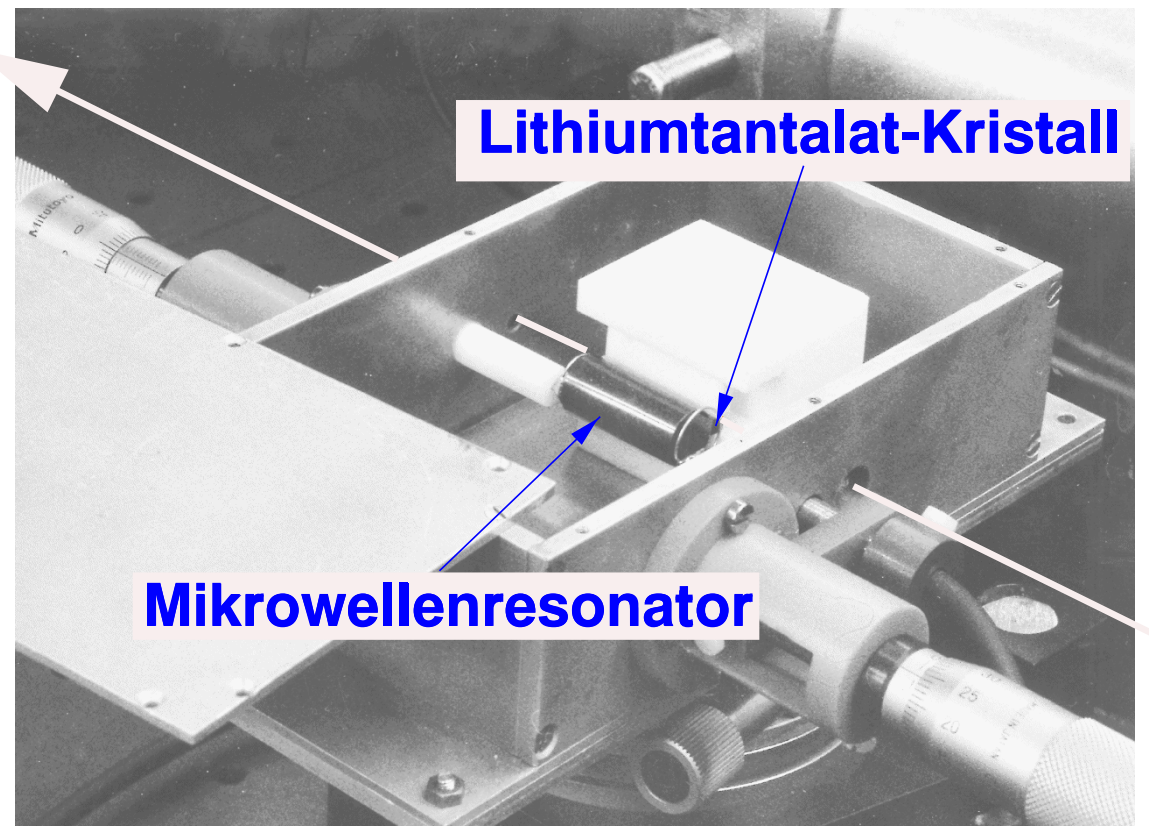

Abb. 3.6: Technischer Aufbau des EOM. Die erste Schleife dient der Einkoppelung der Hochfrequenz; durch Variation ihres Abstands zum Modulator kann die Amplitude des Wechselfeldes im Kristall beeinflußt werden. Der Modulationsindex wird also direkt beeinflußt. Die zweite Schleife schließt den Resonator teilweise kurz und ändert so die Resonanzfrequenz. Der Abstand wird jeweils mit einer Mikrometerschraube eingestellt. Das Betriebsverhalten wird also über die Stellung der beiden Schleifen und die HF-Frequenz gesteuert.

einer Genauigkeit von $100 \mathrm{~Hz}$ oder besser möglich. Die Genauigkeit liegt also deutlich innerhalb der Linienbreite der atomaren Übergänge; eine optische Kontrolle ist nicht erforderlich. Das Auffinden der richtigen Laserwellenlänge kann auf zwei Arten erfolgen :

a. Nach dem in Abschnitt 3.3 beschriebenen Verfahren wird bei ausgeschaltetem Modulator der Übergang $\left|3^{2} S_{\frac{1}{2}}, F=2>\longrightarrow\right| 3^{2} P_{\frac{3}{2}}, F=3>$ aufgesucht. Nach Inbetriebnahme des Modulators wird die Laserfrequenz um etwa $850 \mathrm{MHz}$ erniedrigt und die Fluoreszenz des Na-Strahls optimiert.

b. Ist die Starteinstellung des Lasers nicht bekannt, muß das Fluoreszenzspektrum des Na-Strahls bei Verwendung des Lichts aus dem Modulator durchgefahren werden. Es ergeben sich sieben Fluoreszenzlinien [80], von denen die gewünschte die Stärkste ist. An Stärke und Position der Linie im Spektrum kann diese eindeutig identifiziert werden.

Nach einer Aufwärmzeit von $\frac{1}{2} \mathrm{~h}-1 \mathrm{~h}$, während der häufige Nachjustierung der Parameter (s. Abb. 3.6) notwendig ist, weist der Modulator eine recht gute Langzeitstabilität auf, da geringe Änderungen des Modulationsindex nahezu keinen Einfluß auf die in den ersten Seitenbändern deponierte Leistung haben (s. Abb. 3.4). Damit ist die Langzeitstabilität des Modulators besser als die des Lasers, obwohl keinerlei stabilisierende Elemente eingesetzt werden. 


\section{Weiterer Strahlengang}

Nachdem das Laserlicht den Modulator passiert hat, muß noch ein kleiner Teil ausgekoppelt werden, um kontrollieren zu können, ob Modulator und Laser korrekt arbeiten; außerdem muß der LIF-Detektor versorgt werden. Weiterhin befindet sich ein Teleskop aus einer 150mm- und einer 100mm-Linse im Strahlengang, mit dem der Strahldurchmesser in der Anregungszone angepaßt wird. Beim Einsatz des mechanischen Choppers befindet sich die Chopperscheibe im Focus des Teleskops (s. Abschnitt 3.6); wird die Pockelszelle eingesetzt, ist sie vor dem Teleskop eingebaut. Mit Hilfe dreier Spiegel wird das Licht durch ein Fenster im Kammerdeckel zur Anregungszone geführt.

\begin{tabular}{ll|r} 
Komponente & Zweck & Verlust \\
\hline Modulator & & $10 \%$ \\
Strahlteiler & 7.5 GHz Etalon und Na-Dampflampe & $4 \%$ \\
Strahlteiler & LIF-Detektor & $4 \%$ \\
Teleskop & & $\approx 10 \%$ \\
& \\
Pockelszelle & pseudostatistische & $50 \%$ \\
mit Analysator & Modulation des Lichtes & \\
alternativ: & & $10 \%$ \\
mech. Chopper & Modulation des Lichtes & - \\
2 Spiegel & Umlenkung in die Vakuumkammer & $4 \%$
\end{tabular}

Tab. 3.2: Komponenten im Strahlengang des Dye-Lasers.

\subsection{Na-Anregung und Bestimmung der Primärstrahlgeschwindigkeit}

Nach der Anregungszone durchläuft der Strahl ein Pentaprisma zur senkrechten Umlenkung und wird durch einen in entsprechender Entfernung aufgestellten Spiegel unter $45^{\circ}$ in die Anregungszone zurückgespiegelt (s. Abb. 3.7). Hiernach wird das Licht von einem elektrisch von außerhalb der Vakuumkammer verstellbaren Spiegel in sich zurückgeworfen, um zusätzliche Leistung für die Anregung verfügbar zu machen.

Die in der Anregungszone anfallende Fluoreszenz wird auf ein Faserbündel abgebildet, das das Fluoreszenzlicht einem Sekundärelektronenvervielfacher (SEV) zuführt.

Der Durchlauf unter $45^{\circ}$ bzw. $135^{\circ}$ durch den Natriumstrahl dient der Messung der Strahlgeschwindigkeit unter Ausnutzung des Dopplereffekts. Geht man von einer einheitlichen Strömungsgeschwindigkeit $v$ aus, mißt man die Komponente von $v$ in Richtung des Laserstrahls. Damit ergibt sich für die Differenz zur nicht dopplerverschobenen Anregungsfrequenz 


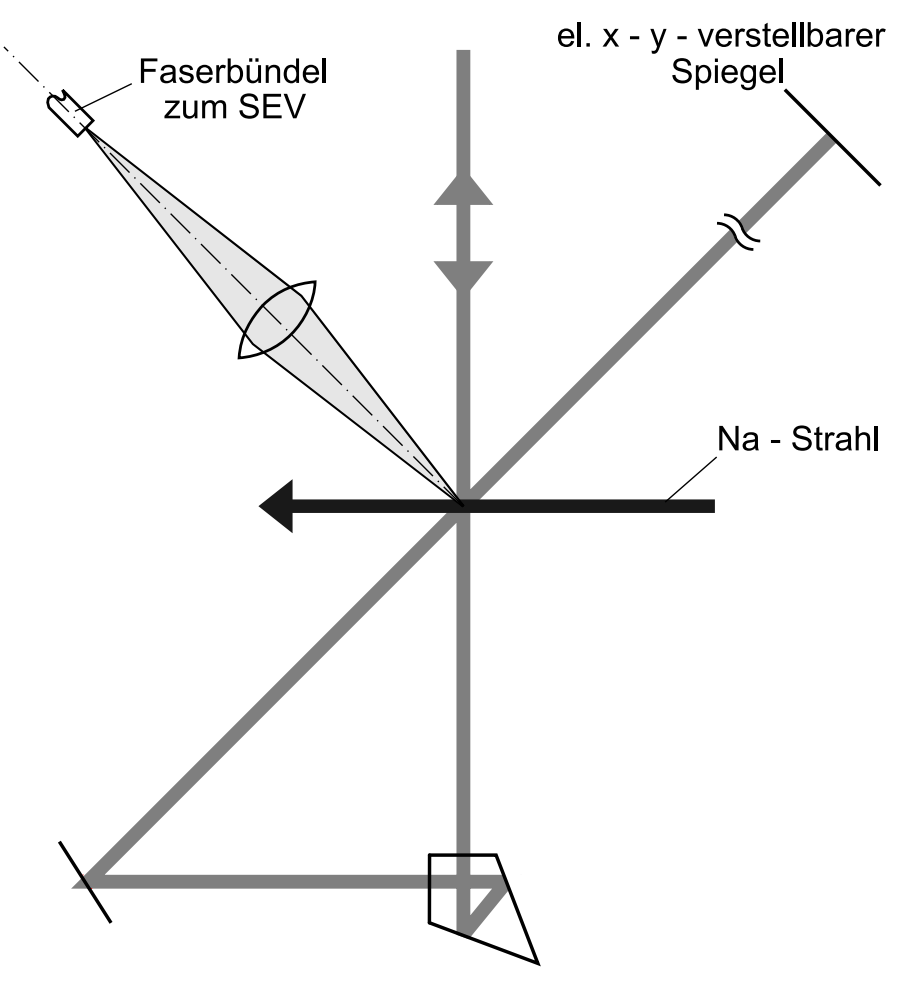

Abb. 3.7: Strahlführung innerhalb der Streukammer.

$$
\Delta v=\frac{ \pm \cos \left(45^{\circ}\right) \cdot v}{\lambda}
$$

da die Anregungszone in beiden Richtungen durchlaufen wird. Die Strahlgeschwindigkeit kann also ermittelt werden, indem die Laserfrequenz über einen hinreichend großen Bereich durchgefahren und das Fluoreszenzlicht gemessen wird. Mit dem maximalen Scanbereich des Lasers wäre eine Geschwindigkeit von $\approx 25000 \frac{\mathrm{m}}{\mathrm{s}}$ erreichbar; dies ist für die erreichbaren Geschwindigkeiten mehr als ausreichend.

Praktisch hat der Natriumstrahl keine einheitliche Geschwindigkeit, sondern eine Geschwindigkeitsverteilung. Aufgrund der sehr guten Kollimation des Strahls ,sieht“die Dopplermessung die Geschwindigkeitsverteilung in Ausbreitungsrichtung, die näherungsweise durch eine Gauß-Funktion gegeben ist. Da der Zusammenhang zwischen $\Delta v$ und $v$ linear ist, können Messungen des Fluoreszenzlichts in Abhängigkeit von der Laserfrequenz direkt in die Geschwindigkeitsverteilung umgeeicht werden. Im auszumessenden Frequenzbereich liegen Übergänge zwischen verschiedenen Hyperfeinniveaus (s. Abb. 2.3). In den üblicherweise gemessenen Spektren wird nur die Aufspaltung im Grundzustand $(\approx 1.8 \mathrm{GHz})$ aufgelöst. Ein solches Spektrum zeigt Abb. 3.8. 


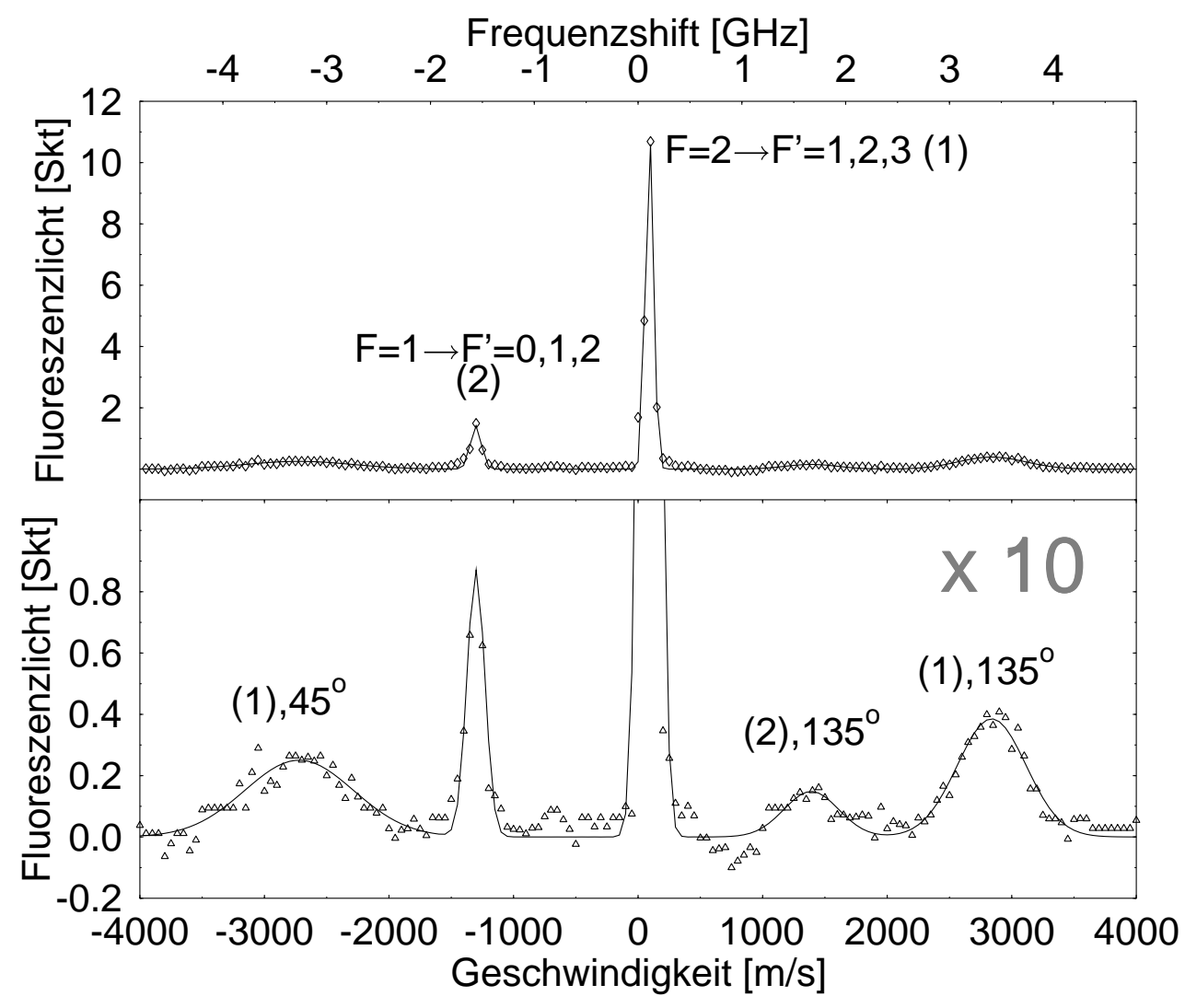

Abb. 3.8: Geschwindigkeitsmessung am Primärstrahl mit dem Dopplereffekt. Die jeweiligen Geschwindigkeiten ergeben sich aus der Differenz zwischen dem Signal des verschobenen und des unverschobenen Überganges.

\subsection{Detektoren}

Zur Detektion der gestreuten Teilchen werden zwei auf verschiedene Prinzipien gegründete Detektoren verwendet: Der eine funktioniert nach dem Verfahren der Oberflächenionisation an einem heißen Faden, der andere nach dem der laserinduzierten Fluoreszenz (LIF). Die grundsätzliche Funktionsweise soll hier in aller Kürze dargestellt werden.

\section{Oberflächenionisations- (Langmuir-Taylor-) Detektor}

Das Grundprinzip eines Oberflächenionisationsdetektors ist, daß beim Auftreffen auf Oberfläche, bestehend aus einem Material hoher Austrittsarbeit, Teilchen geringer Ionisationsenergie ionisiert werden. Die Temperaturabhängigkeit des Vorgangs wird durch die SahaLangmuir-Gleichung

$$
\frac{\text { Anzahl der abgegebenen Ionen }}{\text { Anzahl der abgegebenen Neutralteilchen }} \sim e^{-\left(\frac{I P-W_{a}}{k T}\right)}
$$


mit

$w_{a} \quad$ Austrittsarbeit des Oberflächenmaterials

IP Ionisationspotential der nachzuweisenden Atome

$T$ Oberflächentemperatur

beschrieben. Die Austrittsarbeit des im Experiment verwendeten Oberflächenmaterials Rhenium ist mit $5.17 \mathrm{eV}$ geringfügig größer als das Ionisationspotential des Natriums (5.14 eV) [25].

$\mathrm{Zu}$ beachten ist noch, daß sich die Austrittsarbeit durch Bedampfung der Oberfläche ändert; insbesondere kann die Adsorption von Restgasmolekülen, vor allem von Sauerstoff, die Austrittsarbeit erheblich steigern, während Adsorption des nachzuweisenden Natriums diesen Effekt wieder rückgängig machen kann. In [42] sind diese Effekte an einer Rheniumoberfläche, allerdings für den Nachweis von Lithium und Lithiumiodid untersucht. Für den hier eingesetzten Temperaturbereich ergibt sich dort ein positiver Einfluß der Restgasmoleküle auf die Austrittsarbeit sogar unter Hochvakuumbedingungen, kein Einfluß durch das Alkalihalogenid und ein negativer Einfluß durch Adsorption des Alkalis. Summarisch folgt daraus, daß die Rheniumoberfläche nach kurzer Einstellungszeit eine konstante Austrittsarbeit von $5.4 \mathrm{eV}$ aufweist.

Das im Experiment bei reaktiver Streuung anfallende NaF wird ebenfalls mit dem LangmuirTaylor-Detektor nachgewiesen. Dies ist möglich, da das NaF auf der heißen Oberfläche dissoziiert und das entstehende Natrium ionisiert wird. Nach $[42,45]$ ist die Ionisierungseffizienz für $\mathrm{Na}$ und $\mathrm{NaF}$ bei den verwendeten Fadentemperaturen gleich und beträgt nahezu $100 \%$. Eine Trennung der verschiedenen Spezies durch Oberflächen verschiedener Austrittsarbeit, realisiert durch unterschiedliches Oberflächenmaterial (wie etwa in [68]) oder Bedampfung ([47]) wurde bei dem hier dargestellten Experiment nicht durchgeführt; stattdessen wurde das Natrium selektiv mit laserinduzierter Fluoreszenz nachgewiesen.

Die Durchführung von Laufzeitmessungen stellt gewisse Anforderungen an die Antwortzeit des Detektors. Die Verweildauer der nachzuweisenden Teilchen soll auf der Zeitskala der Laufzeitmessung möglichst vernachlässigbar sein; daraus ergibt sich bei Laufzeiten im Bereich von 50 $\mu$ s bis 500 $\mu$ s für optimalen Betrieb eine Antwortzeit $<1 \mu s$. Die für LangmuirTaylor-Detektoren beobachteten Antwortzeiten liegen im Bereich von $10^{-2}$ bis $10^{-6}$ [4] und hängen wesentlich von der Temperatur ab. Schon aus diesem Grunde muß also eine recht hohe Temperatur gewählt werden. Im gegebenen Experiment konnte die Gesamtantwortzeit des Detektors durch Vergleich mit dem - auf der betrachteten Zeitskala - absolut verzögerungsfreien LIF-Detektor ermittelt werden. Diese Antwortzeit enthält allerdings noch die durch Ionenflugzeit und Massenselektion bestimmte Flugzeit (s. u.). Die Verzögerungszeit zwischen beiden Detektoren beträgt $14 \mu s \pm 3 \mu s$. Da jedoch keine signifikante Verbreiterung der Fluzeitverteilung bei elastischer Streuung im Vergleich der mit beiden Detektoren gemessenen Spektren auftritt, kann diese Verzögerung direkt in die Auswertung übernommen werden. Die Auswirkungen der langen Antwortzeit auf das Auflösungsvermögen werden in Abschnitt 4.3.4 genauer untersucht.

\section{Aufbau und Betrieb des LT-Detektors}

Der im Experiment eingesetzte Detektor besteht aus einem kommerziellen Quadrupol-Massenspektrometer [3], dessen Elektronenstoßionenquelle durch den heizbaren Rhenium-Faden [2] ersetzt ist. 
Die auf dem Faden erzeugten Ionen werden mittels einer Ionenoptik in den Massentrenner fokussiert. Dahinter werden sie umgelenkt zu einem Channeltron [5], welches im Teilchenzählmodus betrieben wird.

Das Massenspektrometer dient dabei ausschließlich der Abtrennung des (Alkali-)Untergrundes. Eine Trennung verschiedener, natriumhaltiger Moleküle ( $\mathrm{NaF}$ bzw. $\left.\mathrm{Na}_{n}\right)$ kann nicht erfolgen, da diese auf dem Faden dissoziiert und als $\mathrm{Na}^{+}$nachgewiesen werden.

Die Ionenquelle befindet sich in einem $\mathrm{N}_{2}$ (fl)-gekühlten Kupferbehälter, um Restgas fernzuhalten. Die Kühlleistung des $\mathrm{N}_{2}$ (fl)-Kreislaufs beschränkt die im Dauerbetrieb maximal mögliche Fadentemperatur. Bei allen hier dargestellten Experimenten betrug die Fadentemperatur $1450^{\circ} \mathrm{C}$ und die des Behälters $-130^{\circ} \mathrm{C}$. Zu Beginn eines Experiments wurde der Faden gereinigt, indem er kurzzeitig einer sehr viel höheren Temperatur ausgesetzt wurde.

\section{Der Monitor-Detektor}

Ebenfalls nach dem Prinzip der Oberflächenionisation arbeitet der Monitordetektor, der sich in der Achse des Primärstrahls jenseits des Streuzentrums befindet. Dieser Detektor dient lediglich der Kontrolle der Primärstrahlintensität und ist dementsprechend unempfindlich ausgelegt. Der LT-Faden ist mit einem zylindrischen Ionenauffänger umgeben, an dem der aufgenommene Ionenstrom von einem Elektrometerverstärker (Cary) gemessen wird. Der Ionenauffänger ist von einer gekühlten Abschirmung umgeben.

\section{Das Bolometer}

In der Achse des Sekundärstrahls befindet sich ein Halbleiterbolometer, das mittels eines evakuierbaren Kryostaten auf die Betriebstemperatur von $1.8 \mathrm{~K}$ gebracht wird. Es kann zur Kontrolle des Sekundärstrahls eingesetzt werden. Hauptsächlich dient es jedoch der Kontrolle des Anregung der Sekundärstrahlmoleküle mit einem $\mathrm{CO}_{2}$-Laser. Dieser wurde für die hier dargestellten Experimente nicht verwendet.

\section{Der LIF-Detektor}

Im LIF-Detektor wird im Prinzip derselbe Anregungsprozess angewendet wie bei der Anregung im Streuzentrum. Allerdings ist hier nicht maximale Anregungseffizienz, sondern ein linearer Zusammenhang zwischen Teilchenzahl und abgegebenem Fluoreszenzlicht anzustreben. Daher muß die Anregungsleistung gering gehalten werden, also eine wesentlich geringere Fluenz eingestellt werden.

Weiterhin besteht die Anforderung, eine dem LT-Detektor vergleichbare Empfindlichkeit zu erreichen. Daher ist eine Lichtsammeloptik erforderlich, die möglichst den gesamten Raumwinkelbereich auf das Nachweissystem abbildet. Dies wird durch ein System von zwei Hohlspiegeln erreicht(s. Abb. 3.9), die zusammen ca. 50 \% des aus dem Nachweisvolumen abgestrahlten Lichts auf ein Faserbündel abbilden.

In [44] befinden sich der exakte technische Aufbau sowie Untersuchungen über die Eigenschaften des LIF-Detektors. Hier sollen nur die für diese Arbeit wichtigen Eigenschaften referiert werden. 
Das zur Versorgung nötige Licht wird außerhalb der Vakuumkammer aus dem Strahl des Dye-Lasers ausgekoppelt und mittels einer multimode-Glasfaser zum Detektor geleitet. Dort wird das Licht nach dem Austritt aus der Faser parallelisiert und durch die Anregungszone in eine Lichtfalle geführt. Im Unterschied zu [44] wurde - durch Einsatz des elektrooptischen Modulators - 2-Farben-Licht verwendet. Infolgedessen wird die eingekoppelte Leistung mit $1 \mathrm{~mW}$ - $2 \mathrm{~mW}$ etwas größer gewählt (s. 4.1). Durch den Betrieb mit 2-Farben-Licht ist die

Verwendung auch bei Messungen von Streuung an $\mathrm{Na}^{2} P \frac{3}{2}$ möglich. Dies wird in 2.4 und genauer in [80] diskutiert.

Die Anzahl der pro nachgewiesenem Atom durchlaufenen Anregungszyklen hängt von dessen Geschwindigkeit ab. Weiterhin ändert schon die zum Nachweis erforderliche Fluenz die Besetzungsverteilung zwischen Grund- und angeregtem Zustand merklich ab. Insgesamt führt die zu einer komplizierten Geschwindigkeitsabhängigkeit, die weder der eines Fluß- noch der eines Dichtedetektors entspricht. Die tatsächliche Geschwindigkeitsabhängigkeit ist prinzipiell durch Simulationsrechnungen zugänglich [44], konnte jedoch für die hier dargestellten Experimente vernachlässigt werden. Die Begründung ist in 4.2.2 gegeben.

Die Empfindlichkeit der LIF-Detektors ist zwar mit der des LT-Detektors vergleichbar, die Untergrundzählrate liegt jedoch eine Größenordnung über der des LTDetektors. Damit sind nicht mehr unter allen Bedin-

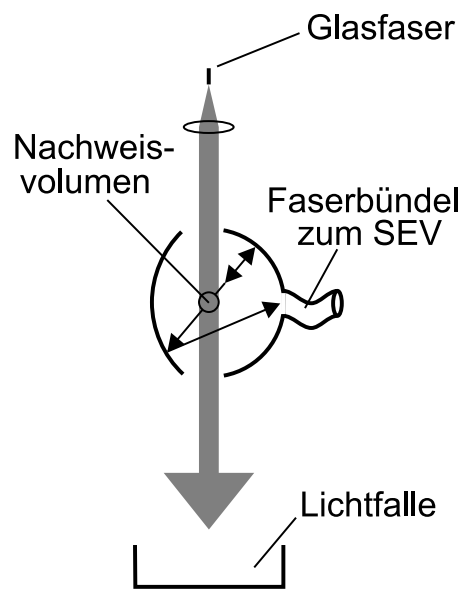
gungen mit dem LIF-Detektor Messungen zu erzielen, unter denen es mit dem LT-Detektor möglich ist.

Der Eintritt der gestreuten Teilchen erfolgt durch eine Blende mit einem Durchmesser von xx mm, die sich im Abstand von xx mm zum Streuzentrum und xx

Abb. 3.9: Schematischer Aufbau des LIF-Detektors. mm von der Detektionszone befindet. Damit wird derselbe Winkel bezüglich des Streuvolumens eingesehen, wie es beim LT-Detektor der Fall ist. Durch eine verschiedene Größe der Detektionsfläche bzw -volumen der beiden Detektoren ergibt sich dennoch ein Unterschied im eingesehenen Raumwinkel, der in der Auswertung durch Normierung der Spektren aufeinander berücksichtigt werden kann.

\subsection{Pseudostatistische Modulation}

Um die pseudo-statistische Laufzeitmessung (s. Abschnitt 2.6 durchzuführen, muß die PseudoRandom-Sequenz auf das Streusignal aufmoduliert werden. Die Modulaton erfolgt im Atomstrahl (für Messungen mit $\mathrm{Na}(3 \mathrm{~S})$ ) bzw. für das Anregungslicht (für Messungen mit $\mathrm{Na}^{*}$ ). Im Falle des Atomstrahls befindet sich ein mechanischer Zerhacker (im folgenden Chopper genannt) $65 \mathrm{~mm}$ vor dem Streuzentrum. Die Scheibe des Choppers (Abb. 3.10) enthält pro Umfang zwei Sequenzen zu je 127 Kanälen sowie - weiter außen - zwei an/aus- (lock in-)Sequenzen. Weiterhin enthält die Scheibe 10 Löcher, die der Drehzahlmessung mittels 
einer Lichtschranke dienen. Das Meßergebnis findet als Eingangsgröße der aktiven Drehzahlstabilisierung Verwendung. Eine weitere Lichtschranke liefert jeweils zu Beginn einer Sequenz einen Startpuls. Der Durchmesser der Scheibe beträgt $10 \mathrm{~cm}$.

Verschiedene konstruktive Merkmale ermöglichen den Betrieb bei hohen Drehzahlen :

- Der Antrieb ist - mit Gummiringen - weich gelagert.

- Die Scheibe besteht aus hochfestem Stahl.

- Der Chopper ist elektronisch ausgewuchtet.

- Das Gehäuse ist wassergekühlt.

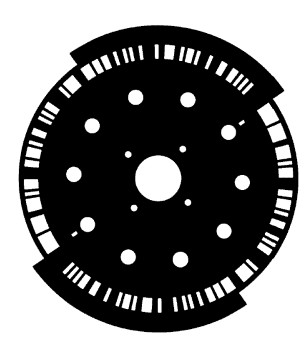

Abb. 3.10: Scheibe des PseudoRandomChoppers

Insgesamt lassen sich so Drehzahlen von bis zu $1000 \mathrm{~Hz}$ erreichen; daraus ergibt sich eine minimale Kanalbreite von $4 \mu s$. Damit läßt sich in der Regel für das TOF-System eine höhere Auflösung erreichen, als sie durch die Apparaturgeometrie und die Strahldaten gegeben ist. Dieser Punkt wird in Abschnitt 4.3 genauer diskutiert.

Wie in Abschnitt 2.6 beschrieben, werden Messungen mit $\mathrm{Na}^{*}$ durchgeführt, indem das Anregungslicht moduliert wird und so des die flugzeitaufgelöste Signaldifferenz zwischen Streuung im angeregten und im Grundzustand gemessen wird. Die Modulation des Laserlichtes wurde für einige Messungen mittels einer Pockelszelle (Electro-Optic Developments, PS 100/2) mit vorgeschaltetem Polarisator und nachgeschaltetem Analysator durchgeführt [15]. Dieser Aufbau ermöglicht das Ein- und Ausschalten des Lichts durch Drehung der Polarisation über die an der Pockelszelle angelegte Spannung, so daß der Analysator durchlässig bzw. undurchlässig wird. Die Pseudo-Random-Sequenz wird elektronisch zur Verfügung gestellt und mit einem speziellen Verstärker auf die für die Pockelszelle notwendige Spannungsdifferenz von 220V transformiert.

Alternativ wurde ein mechanischer Chopper, baugleich dem für den Primärstrahl verwendeten, eingesetzt. Da dieser Chopper an Luft keine ausreichenden Drehzahlen zuläßt, ist er in einer separaten Vakuumkammer auf dem Lasertisch installiert. Chopperkammer und Vakuumpumpe sind dem Lasertisch gegenüber schwingungsisoliert. Da die Breite des Laserstrahls größer ist als diejenige eines Elementarschlitzes des Choppers, muß der Chopper im Brennpunkt eines Teleskops aufgestellt werden. Ein solcher Aufbau wird für die Strahlführung ohnehin benötigt.

Beide Methoden haben Vor- und Nachteile, die hier kurz dargestellt werden sollen :

- Bedingt durch Reflexions- und Absorptionsverluste hat die Gesamtanordnung aus Pockelszelle und Analysator eine maximale Transmission von 50\%. Im Falle des mech. Choppers enstehen nur die Reflexionsverluste an den antireflexbeschichteten Fenstern der Vakuumkammer; es wird eine Transmission von über $95 \%$ erreicht. 
- Eine wesentliche Kenngröße ist der Modulationsgrad

$$
M:=\frac{\text { durchgelassenes Licht bei min. Transmission }}{\text { durchgelassenes Licht bei max. Transmission }} .
$$

Bei der Pockelszelle wird bei niedrigeren Laserleistungen (bis ca. $50 \mathrm{~mW}$ ) vorausgesagte Modulationsgrad von 1\% erreicht, steigt aber bei höheren Leistungen auch nach großem Justieraufwand auf $10 \%$ an. Im Falle des mech. Choppers ist der Laserstrahl „blockiert “und daher $M=0$.

- Die minimale Anstiegszeit für den Lichtpuls beträgt für die Pockelszelle 50ns; diese Zeit ist noch nicht einmal prinzipiell bedingt, sondern wird von der Ansteuerungselektronik verursacht. Damit lassen sich Kanalbreiten unterhalb von $1 \mu s$ realisieren; dies ist mit dem mech. Chopper unmöglich. Allerdings sind bei dem gegebenen Experiment Kanalbreiten von weniger als $4 \mu$ s nicht sinnvoll.

- Der Startzeitpunkt der Sequenz läßt sich für die Pockelszelle von außen vorgeben. Auf der $\mu s$-Zeitskala des Experiments ergeben sich zwischen dem Triggerimpuls und dem Start der Sequenz keine Verzögerungen. Der mech. Chopper kann nicht von außen gesteuert werden, wodurch bei den hier vorgestellten Experimenten jedoch keine Probleme entstanden. Nachteilig ist jedoch, daß der Startzeitpunkt der Sequenz justierabhängig ist. Es ist nur schwer möglich, den Chopper so einzujustieren, daß der Laserstrahl die Sequenz genau an der Position der Lichtschranke durchtritt. Diese Problem läßt sich lösen, indem das Laserlicht hinter dem Chopper auf eine Photodiode gegeben wird und die Verzögerung zum Startimpuls mit einem Oszilloskop gemessen wird. Die gemessene Verzögerung wird dann zusätzlich in die Auswertung eingefügt.

Insgesamt ergibt sich, da in Verbindung mit dem elektrooptischen Modulator eher zuwenig Laserleistung zur Verfügung steht, ein Vorteil für den mech. Chopper. Die in Kap. 6 und 7 dargestellten Experimente mit $\mathrm{SiF}_{4}$ und $\mathrm{NH}_{3}$ wurden mit der Pockelszelle, die Experimente mit $S F_{6}$ (Kap. 5) mit dem mech. Chopper durchgeführt.

\subsection{Meßdatenaufnahme und -verarbeitung}

Am Ausgang des LT- bzw. des LIF-Detektors fallen pro Zählereignis Pulse mit einer Amplitude von $10 \mathrm{mV}$ bis $100 \mathrm{mV}$ und einer Breite von typischerweise $10 \mathrm{~ns}$ an. Bei einer maximalen Zählrate von $150 \mathrm{KHz}$ besteht also keine Gefahr der Überlappung. Die Pulse durchlaufen direkt an der Apparatur einen Vorverstärker. Danach folgt ein Diskriminator, der NIM-Pulse liefert. Diese werden dann an das TOF-Modul weitergegeben.

Das TOF-Modul [38] besteht aus einem Zähler und einem Histogramm-Speicher. Nach dem Startpuls, der vom jeweiligen Pseudorandomchopper zum Beginn der Sequenz geliefert wird, werden die Zählpulse im ersten Kanal des Histogrammspeichers aufsummiert. Nach Ablauf einer Kanalbreite (s. Abschnitt 2.6) wird in den nächsten Kanal umgeschaltet. Dies wird fortgesetzt bis zum nächsten Startpuls, also für die Ablaufzeit einer Sequenz. 
Nach einer gewissen Anzahl Sequenzen enthält der Histogramm-Speicher dann die Detektorfunktion, über eine Anzahl von Durchläufen der Sequenz summiert. Der Inhalt des Histogrammspeichers wird dann in einen Rechner eingelesen und entsprechend Abschnitt 2.6 weiterverarbeitet.

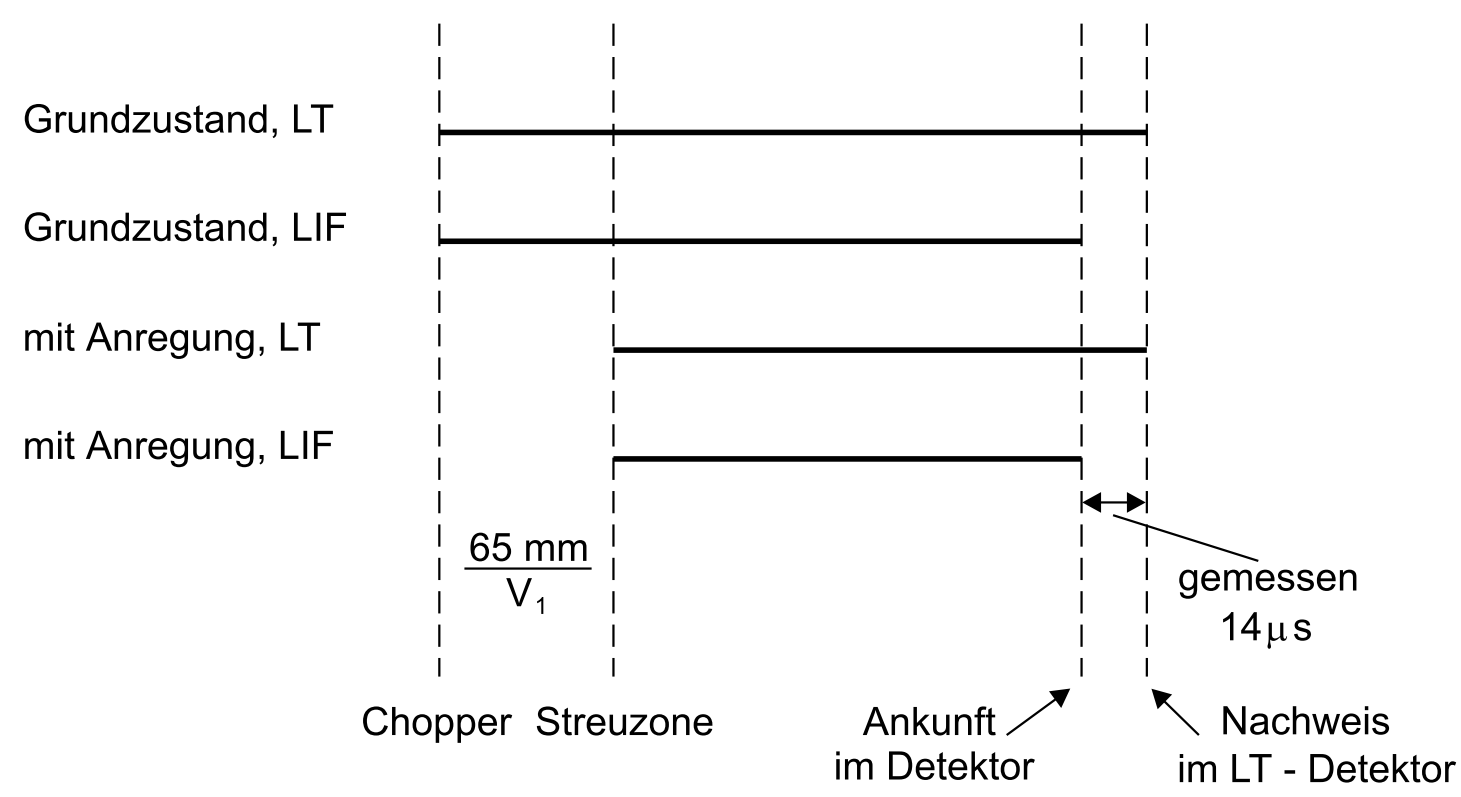

Abb. 3.11: Zeitdiagramm

Bedeutsam ist die Flugzeit zwischen dem Streuprozess und dem Erreichen des Detektors. Je nach experimenteller Konfiguration (s. Abb. 3.11) tragen jedoch weitere Anteile zur Gesamtlaufzeit bei. Die Flugzeit zwischen der Passage des mechanischen Choppers und der Streuzone läßt sich aus der Primärstrahlgeschwindigkeit bestimmen. Problematisch ist dabei jedoch, daß die vom Chopper produzierten „Pakete“auf dieser Strecke aufgrund der Geschwindigkeitsverteilung des Primärstrahls auseinanderlaufen.

Die Zeit zwischen dem Auftreffen der Teilchen auf dem Langmuir-Taylor-Detektor und ihrem Nachweis, bedingt durch die Antwortzeit des LT-Fadens und die Ionenflugzeit bis zum Channeltron, kann durch Vergleich zu unter identischen Bedingungen mit dem LIF-Detektor gemessenen Flugzeitspektren bestimmt werden. Die dem LIF-Detektor inhärenten Verzögerungen sind ausschließlich elektronischer Art und können höchstens im ns-Bereich liegen, sind also vernachlässigbar. Für die Verzögerung des Langmuir-Taylor-Detektors ergibt sich aus diesen Vergleichsmessungen ein Wert von $14 \pm 3 \mu$ s. 


\section{Kapitel 4}

\section{Eigenschaften der verwendeten Apparatur}

\subsection{Anregung im Natriumstrahl}

\subsubsection{Effizienz der Anregung}

Für die Streuung an angeregtem Natrium ist es außerordentlich wichtig, die Anregungseffizienz $\eta$, definiert als

$$
\eta:=\frac{\text { Anzahl der angeregten Na-Atome im Streuvolumen }}{\text { Gesamtzahl der Na-Atome im Streuvolumen }},
$$

zu kennen. Zum einen können so Aussagen über die Qualität des realen Anregungsprozesses gewonnen werden; unabdingbar ist der Wert von $\eta$ für die Bestimmung der Streuquerschnitte im Verhältnis zu den entsprechenden Streuquerschnitten im Grundzustand. Verschiedene Faktoren wirken sich auf die Anregungseffizienz aus:

- Eine nicht vollständige Ausleuchtung des Streuvolumens führt zu einer geometrischen Reduktion von $\eta$. Bei einem (wie im beschriebenen Experiment) nicht sehr genau definierten Streuvolumen ist eine vollständige Ausleuchtung allerdings nicht zu gewährleisten.

- Bei inhomogener räumlicher Leistungsverteilung im Laserstrahl wird in den Randbereichen keine hinreichende Effizienz mehr erreicht.

- In der Zeit bis zur Einstellung einer stationären Verteilung der Zustände herrscht eine geringere Effizienz. Dieser Effekt macht $\eta$ prinzipiell abhängig von der Strahlgeschwindigkeit. Für die verwendeten Strahlgeschwindigkeiten konnte allerdings keine signifikante Abhängigkeit der Anregungseffizienz von der Strahlgeschwindigkeit beobachtet werden. Für hochenergetische Strahlen wird der Effekt bestimmend [26].

- Für Anregung mit nur einer Linie sind nur ${ }^{5} / 8$ aller Atome zugänglich (s. Abschnitt 2.4). Dadurch wird $\eta$ auf max. $31.25 \%$ limitiert. Durch den ,trapping-effect" wird die Effizienz weiter verringert. 


\subsubsection{Methoden der Bestimmung von $\eta$}

Experimentell ist $\eta$ nur schlecht zugänglich. Grundsätzlich wurden verschiedene Wege der Bestimmung von $\eta$ beschritten :

Eine Möglichkeit ist die Messung der Fluoreszenz in Abhängigkeit von der Laserleistung. Die erhaltene Sättigungskurve wird dann durch eine Simulationsrechnung reproduziert [24, 51]. Problematisch ist, daß keine absolute Kalibrierung des Verfahrens möglich ist. Informationen werden nur aus der Kurvenform erhalten. Außerdem müssen in die Simulationrechnung die genauen experimentellen Bedingungen eingehen, die jedoch zum Teil (etwa die räumliche Leistungsverteilung des Lasers) nur sehr schwer zu ermitteln sind [51].

In Streuexperimenten ergibt sich die Möglichkeit, die Anregungseffizienz zu bestimmen, wenn sich im Streusignal Anteile identifizieren lassen, die auf Streuung an $\mathrm{Na}(3 \mathrm{~S})$ beruhen. In [27] ist dies für die inelastische Streuung von $\mathrm{Na}(3 \mathrm{P})$ an $\mathrm{He}^{2+}$ durchgeführt; anhand der Translationsenergie des gestreuten Natriums lassen sich die Eingangskanäle $(\mathrm{Na}(3 \mathrm{~S})$ und $\mathrm{Na}(3 \mathrm{P})$ ) unterscheiden. Es ergibt sich eine Effizienz von $31 \%$ für den zwei-Frequenz-Fall, bei Anregung mit einer Frequenz $11 \%$. Für Stöße zwischen $\mathrm{Na}^{+}$und $\mathrm{Na}(3 \mathrm{~S})$ bzw. $\mathrm{Na}(3 \mathrm{P})$ [71] wurde eine Effizienz von $12 \%$ gefunden. Das im folgenden Abschnitt dargestellte Verfahren, das die reaktive Streuung an $\mathrm{SF}_{6}$ ausnutzt, gehört ebenfalls in diese Gruppe.

Die Messung der Ablenkung des Na-Strahls durch den beim Anregungsprozess insgesamt erfolgenden Impulsübertrag [16] ergab eine Effizienz von ca. $25 \%$.

\subsubsection{Eigene Ergebnisse}

Abb. 4.1 zeigt eine in der verwendeten Apparatur gemessene Sättigungskurve. Für ZweiFrequenz-Anregung (gleichzeitig auf $F=2 \rightarrow F^{\prime}=3$ und $F=1 \rightarrow F^{\prime}=2$ ) zeigt sich qualitativ das erwartete Verhalten: Die Fluoreszenz steigt monoton mit der Leistung und strebt dabei gegen einen Sättigungswert. Es ist nicht sicher, inwieweit der Sättigungswert mit der zur Verfügung stehenden Leistung wirklich erreicht wird. Die Sättigungskurve für die Anregung mit einer Linie (auf $F=2 \rightarrow F^{\prime}=3$ ) zeigt ein Maximum bei etwa $8 m W$ eingestrahlter Leistung und einen Abfall zu größeren Leistungen hin. Daraus läßt sich erkennen, daß der ,trapping-effect“(s Abschnitt 2.4) eine wesentliche Rolle bei höherer Fluenz spielen muß. Der Abfall der Fluoreszenz zu größerer Fluenz hin ist auf diesen zurückzuführen. Im Bereich geringer Fluenz $\left(<30 \frac{\mathrm{mW}}{\mathrm{cm}^{2}}\right)$ führt die Anregung mit einer Linie zu einem größeren Fluoreszenzsignal und damit zu höherer Anregungseffizienz. Dies ist zum einen darauf zurückzuführen, daß ${ }^{1 / 3}$ der gemessenen Leistung nicht auf den richtigen Frequenzen zur Verfügung steht (s. Abschnitt 3.3), zum anderen an der geringeren Übergangswahrscheinlichkeit des $F=1 \rightarrow F^{\prime}=2$-Überganges. Im Limit sehr geringer Fluenz ergibt sich - aus beiden Effekten zusammen - theoretisch eine Reduktion auf $40 \%$. Messungen im LIF-Detektor bestätigen dies [80].

Vergleicht man das maximale Fluoreszenzsignal für Ein- und Zwei-Linien-Anregung, so ergibt sich aus Abb. 4.1 eine Steigerung von $75 \%$. Daraus ergibt sich qualitativ eine deutliche Steigerung der Anregungseffizienz. Quantitativ lassen Streumessungen (s. u.) auf eine Verdreifachung von $\eta$ schließen.

Zur Auswertung der Messungen wird $\eta$ aus Streuexperimenten von $\mathrm{Na}(3 \mathrm{P})$ an $\mathrm{SF}_{6}$ bestimmt. Da es im durchgeführten Experiment nicht möglich ist, das Streuvolumen vollständig 


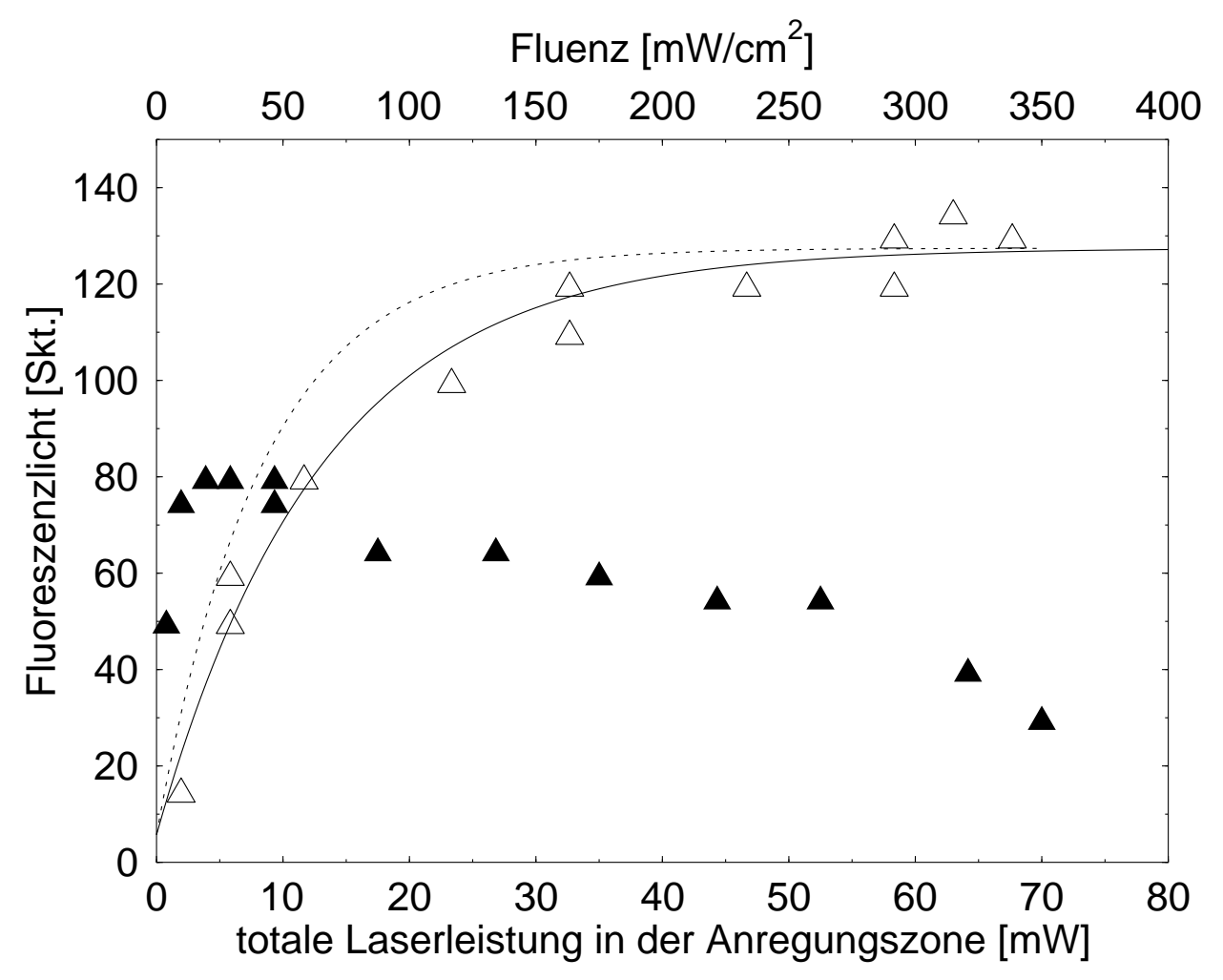

Abb. 4.1: Fluoreszenz in der Streuzone in Abhängigkeit von der eingestrahlten Laserleistung. Bedeutung der Symbole :

$\Delta \quad$ : Anregung mit einer Frequenz

$\Delta \quad:$ Anregung mit zwei Frequenzen

Linie : angepaßte Kurve der Form $A-B e^{-x}$

Strichlinie : angepaßte Kurve, korrigiert auf die zur

Anregung verfügbare Leistung

$(2 / 3$ der totalen $)$

auszuleuchten, soll hier unterschieden werden zwischen der experimentellen Anregungseffizienz $\eta_{\text {exp }}$, bezogen auf alle Na-Atome im Streuvolumen und der nur auf die vom Laser erreichbaren Na-Atome bezogenen ,echten“ Anregungseffizienz $\eta_{e c h t}$.

Die Auswertung der Flugzeitspektren wird in Abschnitt 4.2.2 beschrieben. Hier soll lediglich im Vorgriff dargestellt werden, wie $\eta_{\text {exp }}$ aus den Differenzspektren für das System $\mathrm{Na}(3 \mathrm{P})-\mathrm{SF}_{6}$ extrahiert werden kann. Das aus der reaktiven Streuung an $\mathrm{Na}$ (3P) hervorgehende $\mathrm{NaF}$ weist eine größere kinetische Energie auf als das bei Streuung im Grundzustand entstehende. Die daraus resultierenden Flugzeitverteilungen sind in Abb 4.2 in idealisierter Form dargestellt. Gemessen wird jedoch das Differenzspektrum zwischen dem Zustand mit eingeschaltetem und ausgeschaltetem Laser. Bei eingeschaltetem Laser befinden sich $\eta_{\exp }$ Atome im angeregten und $1-\eta_{\text {exp }}$ Atome im Grundzustand. Damit ergibt sich für das Differenzsignal $I_{\text {diff }}(t)$, zusammengesetzt aus den Anteilen für Grund- und angeregten Zustand $I_{0}(t)$ bzw. $I_{*}(t)$ 


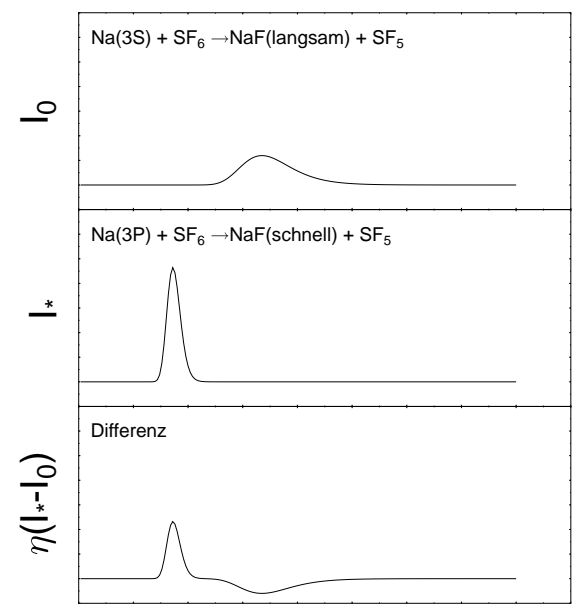

Flugzeit

Abb. 4.2: Zur Bestimmung von $\eta$. Es sind idealisierte Flugzeitspektren der reaktiven Streuung von $\mathrm{Na}$ an $\mathrm{SF}_{6}$ dargestellt (s. Kap. 5); die elastische Streuung ist weggelassen.

$$
I_{d i f f}(t)=\eta_{\text {exp }} I_{*}(t)+\left(1-\eta_{\text {exp }} I_{o}(t)\right)-I_{o}(t)=\eta_{\text {exp }}\left(I_{*}(t)-I_{o}(t)\right)
$$

Sind die Flugzeitverteilungen für $I_{*}$ und $I_{0}$ wirklich zeitlich getrennt, so ist für den Zeitbereich von $I_{0}(t)$, in dem das aus der Streuung im Grundzustand stammende, langsame NaF auftritt, $I_{*}(t)=0$. Gemessen wird also $I_{\text {diff }}(t)=-\eta_{\exp } I_{0}(t)$. Durch eine Messung der Streuung im Grundzustand erhält man $I_{0}$ und damit $\eta_{\text {exp }}$.

Allerdings überlappen in Abhängigkeit von den konkreten Stoßbedingungen die Zeitbereiche von $I_{*}$ und $I_{0}$ in den realen Spektren unter Umständen erheblich. Dadurch ergeben sich Unsicherheiten in der Anpassung der Flugzeitverteilungen und somit eine verhältnismäßig große systematische Ungenauigkeit für $\eta$. Die bei den verschiedenen Experimenten gemessenen Werte für $\eta_{\text {exp }}$ liegen bei $(27 \pm 4) \%$.

Es soll noch der Versuch gemacht werden, den Anteil der Stöße zu ermitteln, die im Bereich der Laseranregung stattfinden. Da der Sekundärstrahl direkt, also ohne Skimmer, verwendet wird, ist die räumliche Verteilung der Streuereignisse im wesentlichen durch die Winkelverteilung des Sekundärstrahls gegeben. Die Dimension senkrecht zum Natriumstrahl wird vernachlässigt, da der ausgeleuchtete Bereich breiter ist als der Strahl. Ebenfalls vernachlässigt wird die räumliche Leistungsverteilung im Laserstrahl, da sie zu einen nur schwer zu ermitteln ist. Für die Dichte $\rho$ auf einer Linie senkrecht zum Strahl ergibt sich aus [54]

$$
\rho(\Theta) \sim \cos ^{2}(\Theta) \cos ^{2}\left(\frac{\pi \Theta}{2 \phi}\right)
$$

$\phi$ ist dabei eine Konstante, abhängig von expandierten Gas, und $\phi$ der Winkel zur Strahlachse. Das Integral dieser Funktion über den ausgeleuchteten Bereich im Verhältnis zum 


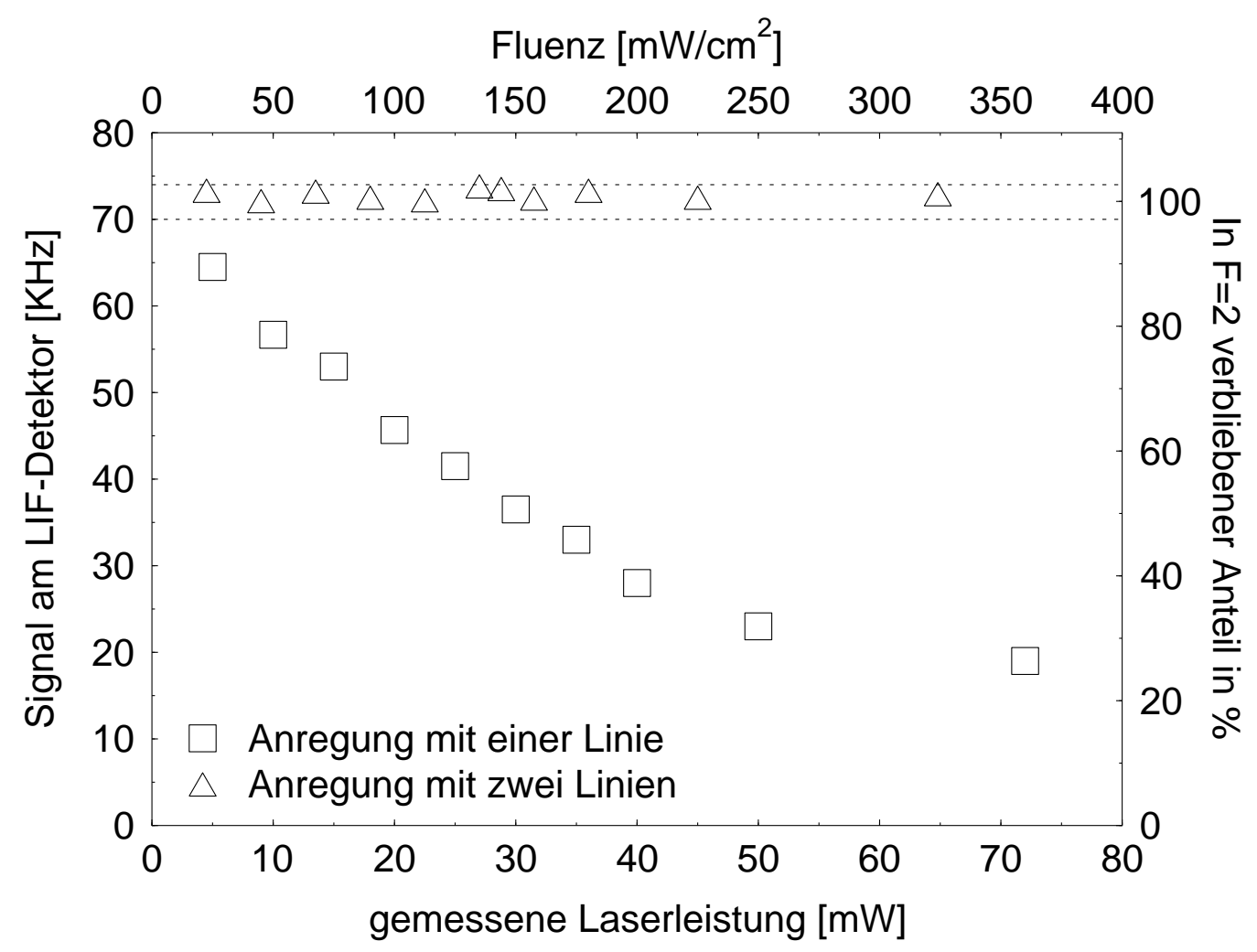

Abb. 4.3: Streusignal am LIF-Detektor in Abhängigkeit von der in der Streuzone eingestrahlten Lichtleistung. Gemessen wird der differentielle Querschnitt von Na-Xe auf dem Laborwinkel $10^{\circ}$. Dieser setzt sich - abhängig von der Lichtleistung - aus einem Beitrag der Streuung im Grundzustand sowie angeregtem Zustand zusammen. Die Differenz zwischen diesen ist jedoch für die Auftragung vernachlässigbar, wie aus Messungen mit dem Langmuir-Taylor Detektor bekannt ist. Die Leistung im LIF-Detektor wurde so angepaßt, daß die Zählrate für die reine Grundzustandsmessung gleich ist.

gesamten zur Streuung beitragenden Bereich ergibt den gesuchten Anteil. Für die im Experiment herrschenden Verhältnisse ergibt sich, daß ca. $80 \%$ der Streuereignisse im ausgeleuchteten Bereich stattfinden. Damit erhält man einen Wert von $34 \%$ für $\eta_{\text {echt }}$.

Beim verwendeten Aufbau wird das Laserlicht sowohl zur Anregung in der Streuzone als auch zur Detektion im LIF-Detektor eingesetzt. Bei Anregung mit einer Linie ist die Nutzung des LIF-Detektors für Messungen mit $\mathrm{Na}(3 \mathrm{P})$ unmöglich, da über den ,trapping-effect“ die F-Besetzungsverteilung im Grundzustand verändert wird. Nach dem Anregungsprozess findet sich ein größerer Anteil der Na-Atome in $F=1$, so daß diese dann für den LIF-Detektor „unsichtbar" werden. Quantitativ ist dieser Effekt in Abb. 4.3 dargestellt. Es zeigt sich, daß schon bei niedriger Fluenz ein nennenswerter Teil des $F=2$-Niveaus entvölkert wird.

Für die Messung mit Zwei-Linien-Anregung ist nicht a priori klar, daß Messungen mit dem LIF-Detektor möglich sind. Aufgrund der verschiedenen Intensitäten der gepumpten Linien ist nämlich eine Änderung der F-Besetzungsverteilung und deren Einfluß auf die Mes- 
sung möglich. Aber Abb. 4.3 zeigt für diesen Fall keine Abhängigkeit von der Fluenz. Dies bedeutet nicht, daß sich die F-Besetzungsverteilung nicht ändert, denn das Zwei-FrequenzLicht wird auch im Detektor verwendet.

\subsection{Auswertung der Flugzeitspektren}

\subsubsection{Typische Spektren}

Für die in dieser Arbeit behandelten Systeme ergeben sich Flugzeitverteilungen, die aus einem bzw. zwei Anteilen bestehen : Ein Anteil beschreibt die für alle Systeme beobachtete elastische bzw. schwach inelastische Streuung. Für die Systeme $\mathrm{Na}(3 \mathrm{~S})-\mathrm{SF}_{6}, \mathrm{Na}(3 \mathrm{P})-\mathrm{SF}_{6}$, $\mathrm{Na}(3 \mathrm{P})-\mathrm{SiF}_{4}$ und $\mathrm{Na}(3 \mathrm{P})-\mathrm{NH}_{3}$ zeigt sich ein auf reaktive Streuung bzw. Löschung zurückzuführender langsamer Anteil. Für die am System Na(3P)-SF 6 gemessenen Spektren ergibt sich durch die Überlagerung mit der Streuung an $\mathrm{Na}(3 \mathrm{~S})$ ein aus drei Anteilen bestehendes Spektrum (Für das Aussehen der Spektren sei auf die entsprechenden Kapitel verwiesen). In keinem der Fälle ist eine Auflösung des Energieübertrages an die einzelnen Vibrationsniveaus des jeweiligen Moleküls möglich, so daß keine innere Differenzierung der einzelnen Anteile erfolgt.

Als direkte Konsequenz ergibt sich, daß ausreichende Information nur durch die Aufnahme der doppelt differentiellen Querschnitte, also der Flugzeitverteilungen, gewonnen werden kann. Die - einfachere - Messung total differentieller Querschnitte ist nicht ausreichend. In den betrachteten Systemen sind die einzelnen Anteile hinreichend gut zeitlich getrennt, so daß eine Bestimmung der Querschnitte über die Flugzeit möglich ist. An Information kann also erhalten werden

- der totale differentielle Querschnitt für den jeweiligen Anteil und

- die mittlere Geschwindigkeit des entsprechenden Anteils.

Wie diese Information aus den Flugzeitspektren erhalten wird, soll im folgenden dargestellt werden. Zusätzlich zur Bestimmung der Geschwindigkeiten ermöglicht die Flugzeitmessung noch die Ermittlung des Untergrundes. Für sehr geringe Geschwindigkeiten gibt es keine Beiträge aus dem Streuprozess mehr; in diesem Bereich werden also nur Untergrundteilchen nachgewiesen, die nicht an der Modulation teilhatten. Dafür muß der Zeitbereich des Flugzeitspektrums so gewählt werden, daß zu einem hinreichend großen Teil des Flugzeitspektrums nur der Untergrund beiträgt.

\subsubsection{Anpassung von Flugzeitverteilungen}

Es wird davon ausgegangen, daß jeder Anteil im Geschwindigkeitsraum durch eine GaußVerteilung

$$
I(v) d v=A e^{\left(-\frac{\left(v-v_{0}\right)^{2}}{\sigma^{2}}\right)} d v
$$


beschrieben werden kann, wobei $v_{0}$ die mittlere Geschwindigkeit und $\sqrt{\ln 2} \sigma$ die Halbwertsbreite ist. Zur Anpassung an die Flugzeitspektren muß $I(v)$ in $I(t)$ transformiert werden (mit $t=\frac{l}{v}, 1$ ist die Flugstrecke zwischen Streuzone und Detektor). Für einen Flußdetektor, dessen Nachweiswahrscheinlichkeit unabhängig von der Geschwindigkeit ist, ergibt sich

$$
d t=\frac{l}{t^{2}} d v
$$

und damit

$$
I(t) d t=A \frac{l}{t^{2}} e^{\left(-\frac{\left(\frac{l}{t}-v_{0}\right)^{2}}{\sigma^{2}}\right)} d t
$$

An die gemessenen Flugzeitverteilungen wird für jeden Anteil eine solche transformierte Gauß-Funktion mit der Marquard-Levenberg-Methode [64] angepaßt. Im exakten Sinne wird die Anpassung von Gauß-Verteilungen hier nur als Methode der Beschreibung benutzt, die gerechtfertigt ist, wenn die Anpassung nach statistischen Kriterien gelingt. Dies ist für die hier vorgestellten Spektren der Fall.

\subsubsection{Extraktion der Querschnitte}

Sind die Gauß-Verteilungen der einzelnen Anteile bestimmt, läßt sich der totale differentielle Querschnitt $Q$ durch Integration über die Zeit bestimmen. Für jede Gauß-Verteilung ist

$$
Q=Q\left(\Theta_{L a b}\right)=\int_{\text {alle } t} I(t) d t=\sqrt{\pi} A \sigma
$$

Damit ergibt sich jeweils ein Paar $\left(v_{m}, Q\left(\Theta_{L a b}\right)\right)$. Für die korrekte Interpretation der Intensitäten ist noch die Transformation ins Schwerpunktsystem durchzuführen. Die mittleren Geschwindigkeiten und die zugehörigen Breiten werden im folgenden in das zugehörige Newton-Diagramm eingetragen, so daß die Verteilung im Labor- wie im Schwerpunktsystem unmittelbar abgelesen werden kann.

Streng genommen ist die Auftragung im Newton-Diagramm nur eine Auftragung im Laborsystem, da für die Betrachtung der Intensitäten im Schwerpunktsystem noch die entsprechende Transformation durchzuführen ist (s. folgender Abschnitt). Bei der Transformation ändern sich auch die Intensitätsverhältnisse innerhalb eines Flugzeitspektrums, also unter Umständen auch die Lage des Maximums und die Breite. Die Interpretation der aufgetragenen Werte im Schwerpunktsystem stellt damit nur eine (gute) Näherung dar, die im Falle beliebig guter Apparaturauflösung korrekt ist (s. Abschnitt 4.3).

Die Alternative wäre die Anpassung eines ,geratenen“" Querschnittsmodells $Q\left(\Theta_{C M}, v_{C M}\right)$, bis dessen Rücktransformation ins Laborsystem die gemessenen Daten wiedergibt und die Darstellung von $Q\left(\Theta_{C M}, v_{C M}\right)$ als Contourdiagramm (z. B. in [68, 45]). Eine solche Darstellung bietet den Vorteil, daß Intensitäts- und Geschwindigkeitsverteilung in einem Diagramm ablesbar sind, während sich die hier gewählte Auftragung an der Darstellung der tatsächlich gemessenen Daten orientiert.

Insgesamt werden die Meßdaten also dargestellt als 
Newton-Diagramm, aus dem sich die Geschwindigkeitsverteilung im Labor- wie im Schwerpunktsystem ablesen läßt, das jedoch keine Intensitätsinformation enthält sowie als

Winkelverteilung im Schwerpunktsystem, die den totalen differentiellen Querschnitt wiedergibt.

\subsection{Kinematik und Auflösungsvermögen}

\subsubsection{Transformation vom Labor- ins Schwerpunktsystem}

Die gemessenen Flugzeitspektren bestehen aus einer Anzahl von Meßpunkten, die jeweils die zu einem Winkel und einer Geschwindigkeit im Laborsystem zugehörige Intensität angeben. Physikalisch relevant ist jedoch die Verteilung im Schwerpunktsystem. Im Newtondiagramm (s. Abschnitt 2.3) beschreibt der Vektor $\vec{v}_{1}^{\prime}$ den Winkel $\theta_{\text {lab }}$ und die Geschwindigkeit $v_{l a b}$ im Laborsystem. $\vec{u}_{1}^{\prime}$ ist die entsprechende Größe im Schwerpunktsystem; Winkel und Geschwindigkeit seien hier $\theta_{c m}$ und $v_{c m}$ genannt. Mit der Schwerpunktsgeschwindigkeit $\vec{c}$ ist

$$
\vec{v}_{1}^{\prime}=\vec{c}+\vec{u}_{1}^{\prime}
$$

woraus sich $\theta_{c m}$ und $v_{c m}$ in Abhängigkeit von $\theta_{l a b}$ und $v_{l a b}$ bei gegebener Primär- und Sekundärstrahlgeschwindigkeit und den entsprecheden Teilchenmassen berechnen lassen (Abschnitt 2.3).

Zusätzlich zu dieser Koordinatentransformation muß noch beachtet werden, daß - aufgrund der Breite des Detektors - die Intensität über ein bestimmtes Raumwinkelelement $d \Omega$ integriert wird. Mit der Transformation ins Schwerpunktsystem geht ein Wechsel der Integrationsvariable vom Labor- auf den Schwerpunktswinkel einher. Als Jacobi-Determinante dieser Transformation ergibt sich das Verhältnis der entsprecheden Raumwinkelelemente $\frac{d \omega}{d \Omega}$. Damit ist die transformierte Intensität

$$
I\left(\theta_{c m}, v_{c m}\right)=I\left(\theta_{l a b}, v_{l a b}\right) \frac{d \omega}{d \Omega}
$$

Für $\frac{d \omega}{d \Omega}$ ergibt sich abhängig von der Art der Streuung $\frac{v_{1}^{\prime 2}}{u_{1}^{\prime 2} \cos \zeta}$ (für elastische Streuung bzw. inelastische/reaktive Streuung, wenn diskrete Niveaus aufgelöst werden) oder $\frac{v_{1}^{\prime 2}}{u_{1}^{\prime 2}}$, wenn eine Mittelung über eine kontinuierliche Energieverteilung für inelastische/reaktive Streuung ausgeführt wird [8].

Für die praktische Auswertung in einem Experiment mit realen Strahlprofilen und Geschwindigkeitsverteilungen ergibt sich das Problem, daß ein bestimmtes Paar von $\theta_{l a b}$ und $v_{l a b}$ nicht eindeutig mit entsprechenden $\theta_{c m}$ und $v_{c m}$ verbunden ist (Prinzipiell existiert noch die Möglichkeit, daß der Newtonkreis zweifach geschnitten wird. Dieses Problem stellt sich in der vorliegenden Arbeit nicht.). Sind die Voraussetzungen für den Grenzfall sehr großen Auflösungsvermögens nicht gegeben, wird bei der Messung über viele verschiedene Newton-Diagramme gemittelt. Dieses hat zwei Konsequenzen : 
- Das Auflösungsvermögen, das in der Möglichkeit besteht, verschiedene Schwerpunktswinkel und -geschwindigkeiten zu trennen, wird beeinträchtigt.

- Die Transformation vom Labor- ins Schwerpunktsystem wird im mathematisch exakten Sinne unmöglich.

Es existieren verschiedene Ansätze, dennoch eine Auswertung der experimentellen Daten vorzunehmen:

(a). Unter Umständen kann eine Entfaltung der experimentellen Daten erfolgen [75], die es dann ermöglicht, konstruktiv die Daten im Schwerpunktsystem aus denen im Laborsystem zu errechnen.

(b). Aus einer vorgegebenen Geschwindigkeits- und Winkelverteilung im Schwerpunktsystem kann unter Berücksichtigung aller Mittelungseffekte durch Simulation die Verteilung im Laborsystem errechnet werden [13]. Diese wird dann mit den experimentellen Daten verglichen.

(c). Bei hinreichend hoher Auflösung der Apparatur werden alle Mittelungseffekte vernachlässigt und die Transformation wird mit den Mittelwerten der entsprechenden Daten (z. B. Strahlgeschwindigkeiten) vorgenommen.

Die hier verwendete Vorgehensweise stellt eine Kombination von (b) und (c) dar. Aufgrund des für die meisten Messungen sehr asymmetrischen Newton-Diagramms (bis auf $\mathrm{Na} \leftrightarrow \mathrm{NH}_{3}$ ), in dem die Moleküle für die Natriumatome fast ein ,stehendes“ Target darstellen, ist die Winkelauflösung der Messungen relativ hoch, so daß die Vernachlässigung der Mittelungseffekte erwägenswert erscheint. Diese Argumentation soll im folgenden durch Simulationsrechnungen und den Vergleich mit Messungen elastischer Streuung am System $N a \leftrightarrow X e$ gestützt werden. Diese Simulationsrechnungen ermöglichen gleichzeitig Aussagen über das Auflösungsvermögen.

\subsubsection{Behandlung von Mittelungseffekten durch Simulation}

Um die Rücktransformation einer gegebenen Winkel- und Geschwindigkeitsverteilung auszuführen, muß eine gewichtete Mittelung über alle möglichen Newton-Diagramme ausgeführt werden. Hierzu muß über folgende Größen integriert werden :

- die Geschwindigkeits- und Intensitätsverteilung der beiden Strahlen, gegeben durch eine Dichte $n\left(\vec{v}_{x}\right)$, wobei der Geschwindigkeitsvektor $\vec{v}_{x}$ Betrag und Richtung der jeweiligen Teilchen beschreibt,

- das vom Detektor eingesehene Raumwinkelelement, beschrieben durch einen Winkel $\Theta$ in der Streuebene und $\Phi$ senkrecht dazu sowie

- den Geschwindigkeitsvektor $\vec{u}$ des gestreuten Teilchens mit seiner Winkel- und Geschwindigkeitsverteilung. 


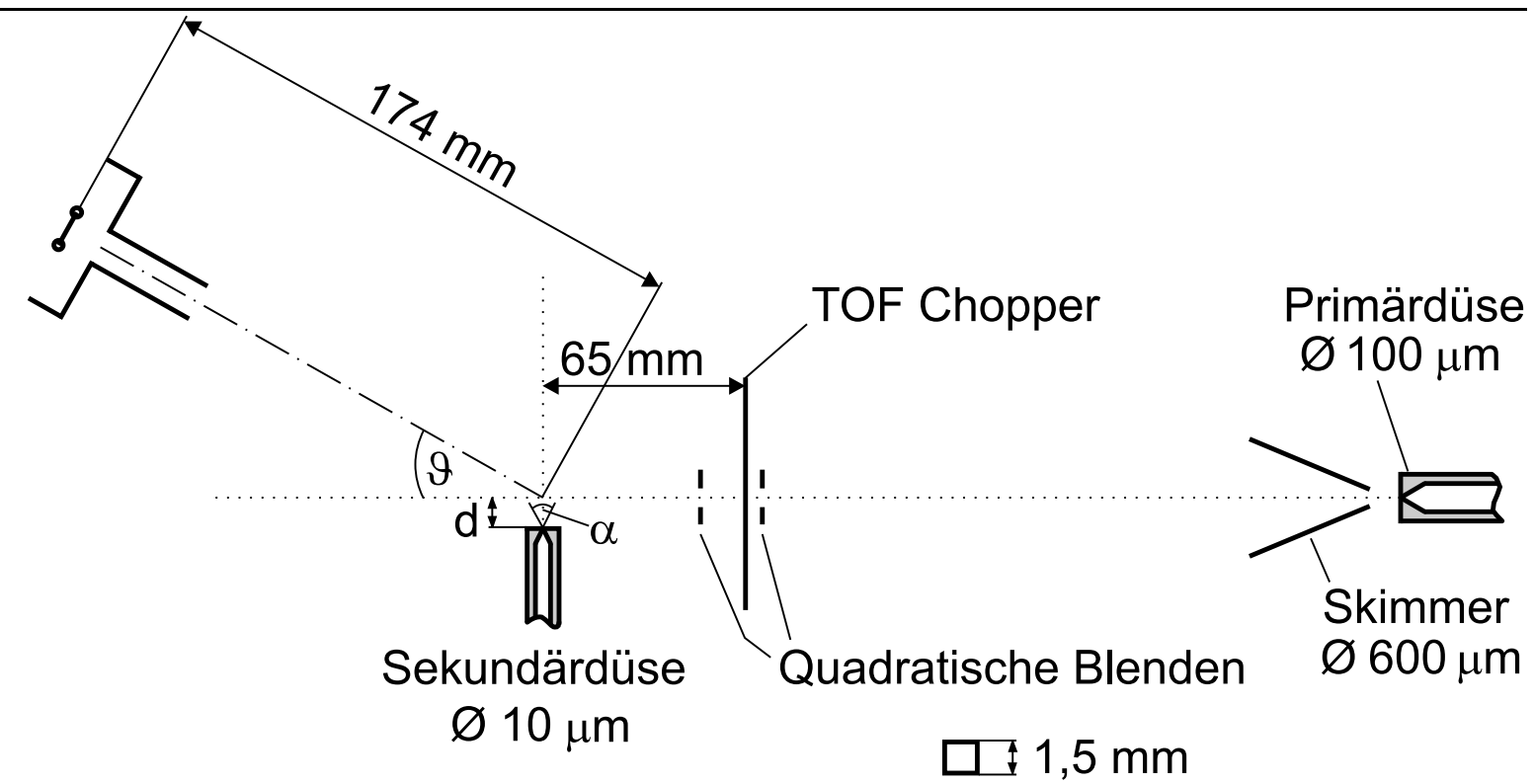

Abb. 4.4: Wesentliche geometrische Apparaturparameter.

Damit ist insgesamt ein 11-dimensionales Integral auszuwerten (je 3 Komponenten für $n\left(\vec{v}_{1}\right), n\left(\vec{v}_{2}\right)$ und $\vec{u}$ sowie $\Theta$ und $\left.\Phi\right)[13]$.

Für die gegebene Apparatur sollen nur diejenigen Größen in die Berechnung einfließen, die von erheblicher Bedeutung sind. Die bedeutsamen Größen sind in Abb. 4.4 dargestellt. Da der Natriumstrahl sehr gut kollimiert ist, kann dessen Winkelverteilung vernachlässigt werden. Ebenso kann Streuung außerhalb der Streuebene außer acht gelassen werden. Für den Sekundärstrahl wird davon ausgegangen, daß die räumliche Intensitäts- und die Geschwindigkeitsverteilung separat betrachtet werden können.

Damit verbleiben als Parameter

- die Geschwindigkeitsverteilungen von Primär- und Sekundärstrahl, für die jeweils eine Gauß-Verteilung verwendet wird (s. Abschnitt 2.5),

- die Winkelverteilung des direkt, d. h. ohne Skimmer, in die Streuzone gerichteten Sekundärstrahls, die durch eine $\cos ^{2}$-Verteilung [54] gegeben ist sowie

- der Einsichtswinkel des Detektors in der Streuebene.

Die numerische Berechnung der Rücktransformation geschieht mit einem Monte-CarloSimulations-Programm. Dabei werden für die Parameter zufällige Werte entsprechend ihrer Verteilung ausgewählt bzw. nachträglich das statistische Gewicht festgestellt. Für einen solchen Streuprozess werden dann mittels des Newton-Diagramms der Streuwinkel im Laborsystem sowie die Gesamtflugzeit festgestellt. Dabei können noch die zusätzliche Flugstrecke zwischen dem mechanischen Chopper und der Streuzone sowie Verzögerungen im Detektor berücksichtigt werden. Die Sammlung der Daten erfolgt in einem zweidimensionalen Histogramm, dessen Bins jeweils zeitlich einen Flugzeitkanal und im Winkel eine Detektorbreite 


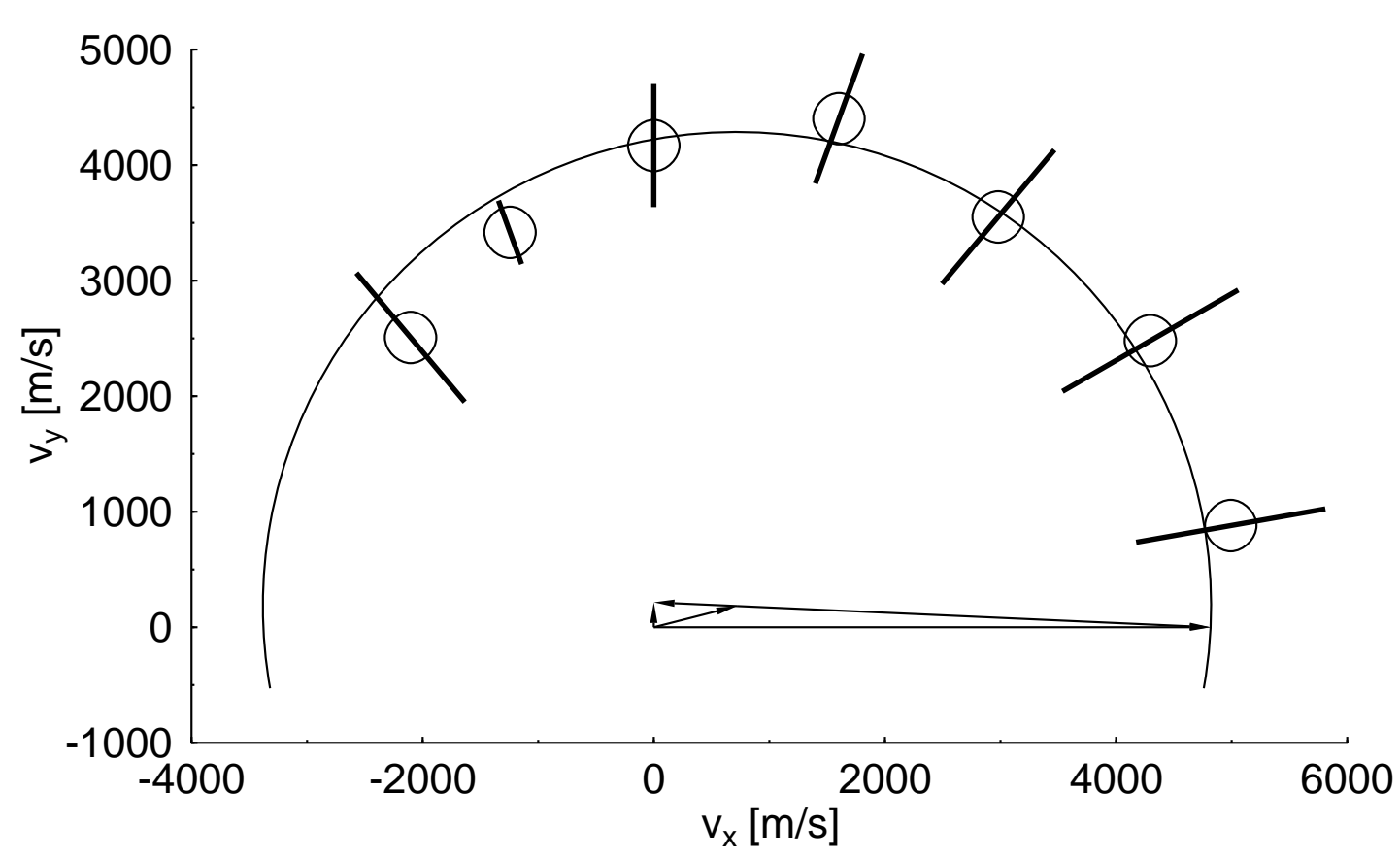

Abb. 4.5: Newtondiagramm der Streuung von $\mathrm{Na}$ an Xe bei einer c.m.-Stoßenergie von $2.4 \mathrm{eV}\left(v_{N a}=\right.$ $\left.4800 \frac{m}{s}, v_{X e}=266 \frac{m}{s}\right)$. Die Symbole geben die jeweils wahrscheinlichste Geschwindigkeit, die Balken die Halbwertsbreite der Flugzeitspektren im Geschwindigkeitsraum wieder.

erfassen. Dieses Verfahren wird hinreichend oft wiederholt; es hat sich gezeigt, daß ab etwa $10^{7}$ Streuereignissen keine Änderung der berechneten Verteilung mehr erfolgt.

\subsubsection{Testmessungen am System $\mathrm{Na}-\mathrm{Xe}$}

Um die Eigenschaften der verwendeten Apparatur zu testen, wurden Testmessungen elastischer Streuprozesse durchgeführt. Da bei elastischer Streuung die Geschwindigkeit im Schwerpunktsystem nach der Streuung derjenigen vor der Streuung gleich ist, ist die Geschwindigkeit der gestreuten Teilchen (im Gegensatz zu inelastischer bzw. reaktiver Streuung) a priori bekannt. Die Messungen dienen dazu, festzustellen,

- wie das bekannte Newton-Diagramm in der gegebenen Streugeometrie wiedergegeben wird,

- in welchem Maße inelastische Prozesse, die nur eine kleine Abweichung vom elastischen Verhalten darstellen, aufgelöst werden können sowie

- dem Vergleich mit simulierten Geschwindigkeitsverteilungen.

Um die Messungen unter kinematisch ähnlichen Bedingungen auszuführen wie die überwiegend ausgeführten Messungen mit $\mathrm{SF}_{6}$ und $\mathrm{SiF}_{4}$, wurde als Targetgas Xenon gewählt, 


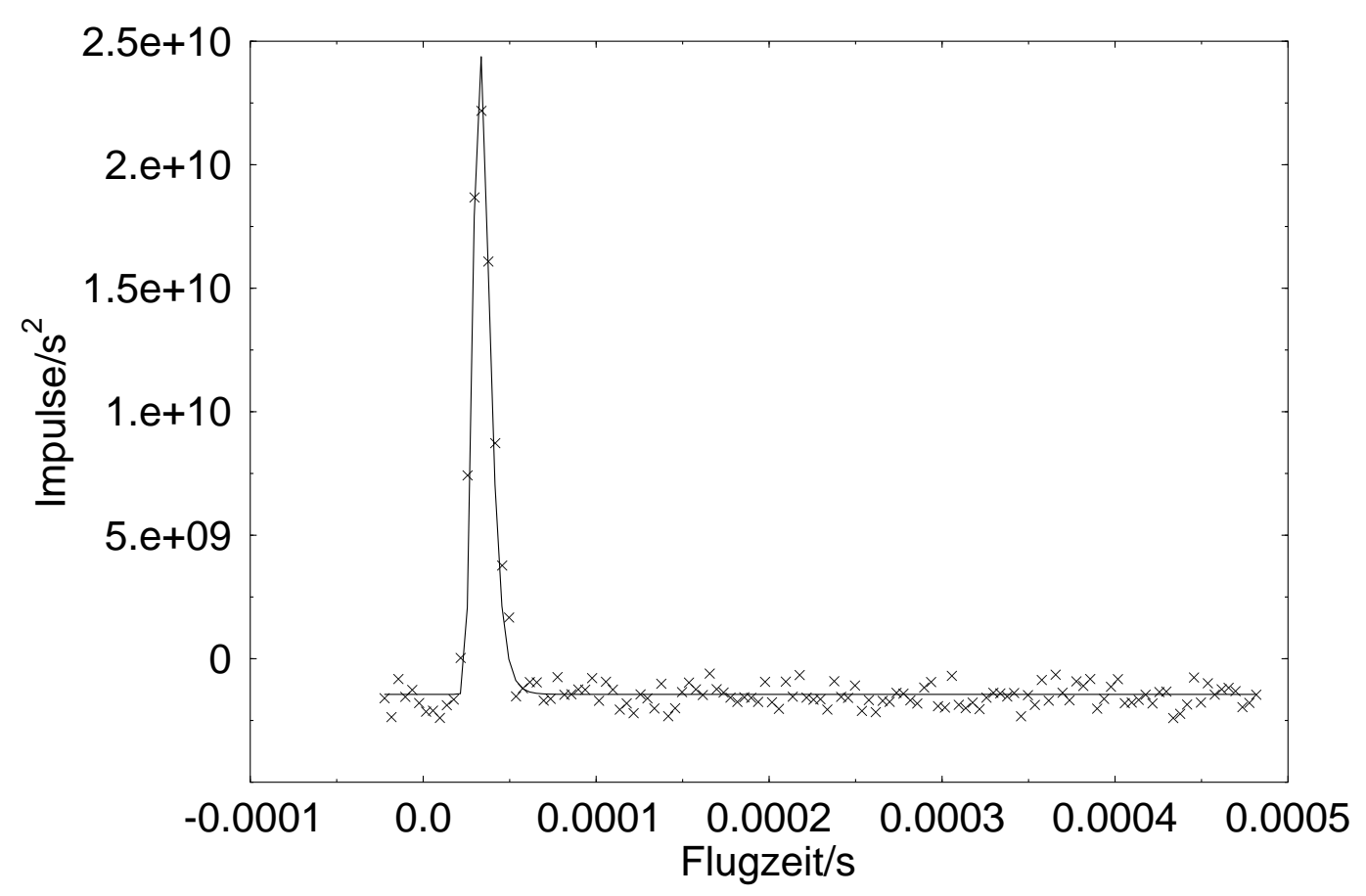

Abb. 4.6: Flugzeitspektrum Na-Xe auf $\theta_{l a b}=10^{\circ}$ im Vergleich mit dem aus der Simulation erhaltenen Spektrum. Um den Vergleich der Spektren zu ermöglichen, ist das simulierte Spektrum, das ebenso wie das gemessene 127 Flugzeitkanäle enthält, als durchgezogene Linie dargestellt. Es wurde ausschließlich eine Normierung der Intensität vorgenommen.

das mit einer Masse von $131 \mathrm{u}$ nahe bei $\mathrm{SF}_{6}(146 \mathrm{u})$ liegt.

Abb. 4.5 zeigt eine Messung mit verhältnismäßig hoher Stoßenergie. Für das zugrundeliegende Newtondiagramm wurde die Natrium-Geschwindigkeit mit der Laser-DopplerMethode gemessen, die Geschwindigkeit des Xe berechnet. Die Maxima der Flugzeitverteilungen liegen innerhalb der Fehlergrenzen auf dem theoretischen Kreis. Die geringen Abweichungen zu größeren Geschwindigkeiten für kleine Winkel und kleinere Geschwindigkeiten für große Winkel zeigen sich auch für entsprechende simulierte Spektren. Die eingetragenen Halbwertsbreiten der Flugzeitverteilungen, die nicht aus der Physik des Streuprozesses stammen, sondern ausschließlich auf die im vorigen Abschnitt aufgeführten Parameter zurückzuführen sind, geben Hinweise auf das Auflösungsvermögen bezüglich der Geschwindigkeit.

Ein gemessenes Flugzeitspektrum ist - gemeinsam mit dem Ergebnis des Simulationsprogramms - in Abb. 4.6 zu sehen. Das in der absoluten Intensität normierte simulierte Spektrum gibt das gemessene korrekt wieder.

Für die gegebene Situation soll noch die in 4.2.2 dargestellte Methode der Auswertung untersucht werden. Zu diesem Zweck wird die Wirkung der Auswerteprozedur auf Meßdaten untersucht, deren Winkelverteilung im Schwerpunktsystem a priori bekannt ist. Diese 
„Meßdaten“ werden für die kinematischen Bedingungen des Systems $\mathrm{Na}-\mathrm{Xe}$ unter Verwendung eines isotropen Querschnitts mittels der Monte-Carlo-Simulation erzeugt. Da die gemessenen Flugzeitverteilungen korrekt reproduziert werden (Abb. 4.6), wird davon ausgegangen, daß die kinematischen Verhältnisse korrekt beschrieben werden. Mit den durch das Simulationsprogramm erzeugten Flugzeitverteilungen wurde die beschriebene Auswertung durchgeführt. Die als Ergebnis erhaltene Winkelverteilung im Schwerpunktsystem ist nicht ganz konstant, erweist sich aber als gute Näherungslösung.

\subsubsection{Auswirkungen auf das Auflösungsvermögen}

Das Auflösungsvermögen des verwendeten experimentellen Aufbaus teilt sich für die Messung doppelt differentieller Querschnitte in eines bezüglich des Streuwinkels und eines bezüglich der Geschwindigkeit der gestreuten Teilchen auf. Folgende Einflußfaktoren bestimmen das Auflösungsvermögen :

(a). Alle in Abschnitt 4.3.2 betrachteten Faktoren, im wesentlichen Geschwindigkeits- und Winkelverteilung von Primär- und Sekundärstrahl wirken sich sowohl im Streuwinkel als auch in der Flugzeit auf das Auflösungsvermögen aus.

(b). Die Antwortzeit des Detektors und

(c). die Kanalbreite des TOF-Systems wirken sich in erster Näherung nur auf die Auflösung bezüglich der Geschwindigkeit aus.

(d). Prinzipiell spielt noch die Bestimmungsgenauigkeit geometrischer Größen, etwa der Position des Winkeldetektors eine Rolle. Praktisch ergibt sich jedoch kein Einfluß auf des Auflösungsvermögen.

Da die physikalisch entscheidenden Größen Streuwinkel und Geschwindigkeit im Schwerpunktsystem sind, wird das Auflösungsvermögen als Fähigkeit betrachtet, verschiedene Werte dieser Größen im Experiment zu trennen. Aus (a) ergibt sich, daß das Auflösungsvermögen nicht nur durch die verwendete Streuapparatur bestimmt wird, sondern auch zu den Eigenschaften des untersuchten Streusystems gehört. Aus dem Newton-Diagramm des jeweiligen Systems lassen sich generelle Trends für das zu erwartende Auflösungsvermögen ableiten. Im Falle eines möglichst ,asymmetrischen“ Newton-Diagramms, also für einen schnellen Primärstrahl aus leichten Teilchen, gekreuzt mit einem langsamen Sekundärstrahl einer schweren Spezies, ergibt sich ein großes Auflösungsvermögen bezüglich des Winkels für den Nachweis der Primärstrahlteilchen, da sich ein bestimmtes Intervall von Schwerpunktswinkeln über einen großen Bereich von Laborwinkeln erstreckt (s. Abb. 2.2). Für den Nachweis der Sekundärteilchen gilt entsprechend das Gegenteil. Für inelastische und reaktive Streuung verringert sich das Auflösungsvermögen mit geringer werdender Geschwindigkeit der gestreuten Teilchen. Für die Systeme $\mathrm{Na}-\mathrm{SF}_{6}, \mathrm{Na}-\mathrm{SiF}_{4}$ und $\mathrm{Na}-\mathrm{Xe}$ liegen die Verhältnisse also günstig; daher ist die Verwendung eines unkollimierten Sekundärstrahl bei annehmbarer Winkelauflösung möglich.

Im günstigsten untersuchten Fall, der am System Na-Xe durchgeführten Testmessung, läßt sich durch Simulationsrechnungen ein Auflösungsvermögen von ca. $2^{\circ}$ über den gesamten Winkelbereich ermitteln. Das Ergebnis für $\Theta_{c m}=20^{\circ}$ ist in Abb. 4.7 dargestellt. 


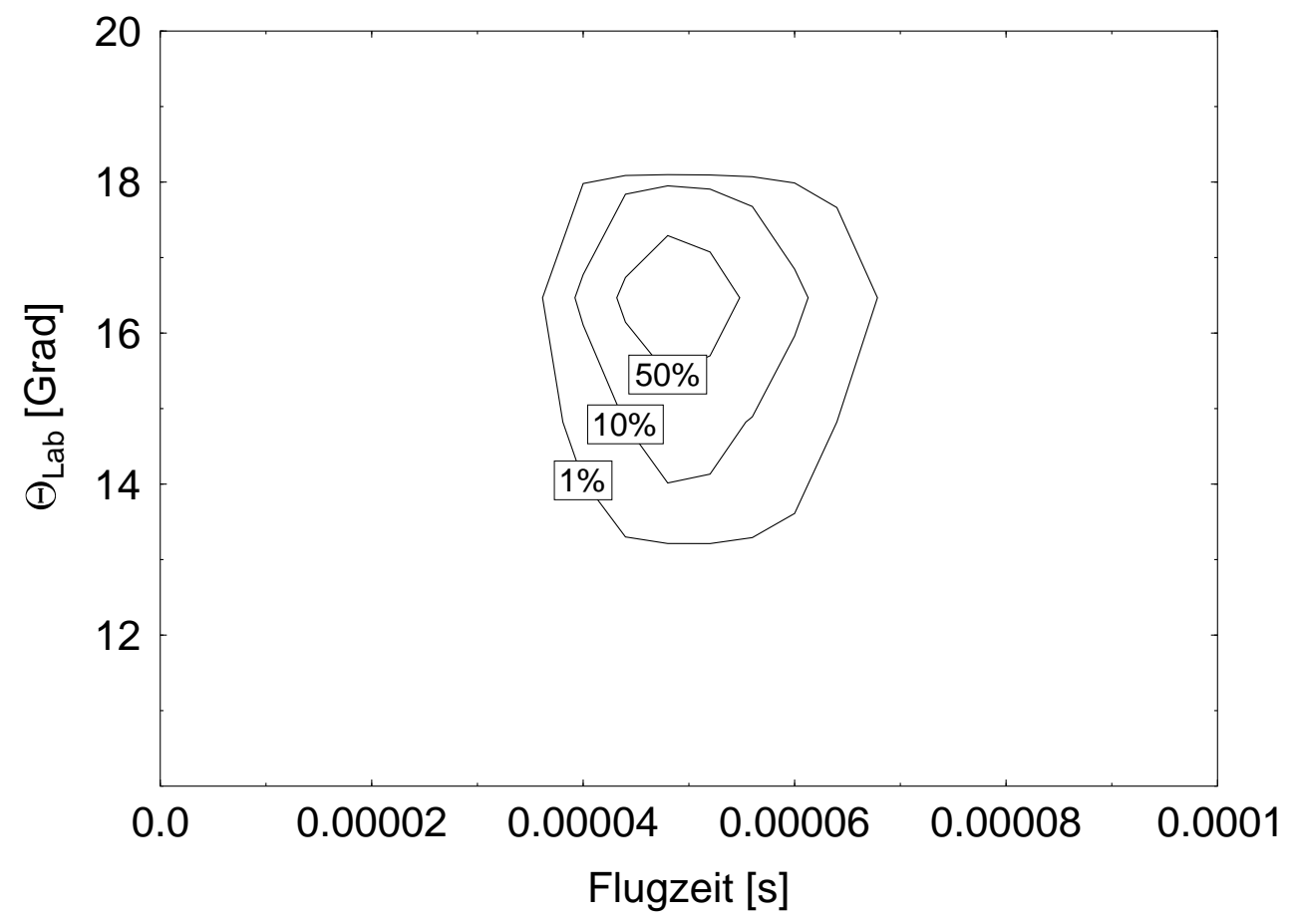

Abb. 4.7: Simuliertes Experiment für Streuung ausschließlich auf $\Theta_{c m}=20^{\circ}$. Die sonstigen Daten entsprechen denen des Na-Xe-Experiments, das im vorigen Abschnitt dargestellt ist. In der Höhe aufgetragen ist die Intensität im Laborsystem (Maximum $=100 \%$ ). Aus der Halbwertsbreite an der Position der mittleren Geschwindigkeit läßt sich das Auflösungsvermögen entsprechend dem Rayleigh-Kriterium zu 1, $8^{\circ}$ bestimmen.

Für die in den anderen Systemen beobachteten reaktiven bzw. inelastischen Anteile ergibt sich ein schlechteres Auflösungsvermögen von typischerweise $5^{\circ}$ bis hinunter zu $10^{\circ}$ für die

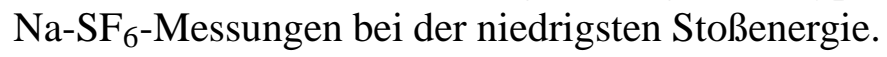

Die Einflußfaktoren (b) und (c) wirken sich im wesentlichen nur auf das Auflösungsvermögen bezüglich der Geschwindigkeit aus. Die Antwortzeit das Detektors stellt dabei die kleinste sinnvolle Kanalbreite des TOF-Systems dar. Die minimal mögliche Kanalbreite von $4 \mu s$ liegt im Bereich der Antwortzeit des Langmuir-Taylor-Detektors.

Da die primäre Meßgröße die Flugzeit, die gewünschte Größe jedoch die Geschwindigkeit $v \sim \frac{1}{t}$ ist, ergibt sich eine mit steigender Geschwindigkeit sinkende Auflösung, das Auflösungsvermögen des TOF-Systems ändert sich also innerhalb des Spektrums(Abb. 4.8).

Im Experiment wurden verschiedene Kanalbreiten verwendet : Da bei größerer Kanalbreite mehr Ereignisse im jeweiligen Kanal aufgesammelt werden, ergibt sich ein besseres Signal/Rausch-Verhältnis im entsprechenden Flugzeitspektrum bzw. es ist eine geringere Meßzeit notwendig (Natürlich läßt sich der Effekt auch durch nachträgliche Zusammenfassung der Kanäle erreichen.). Es ist abzuwägen, ob der durch vergrößerte Kanalbreite ver- 


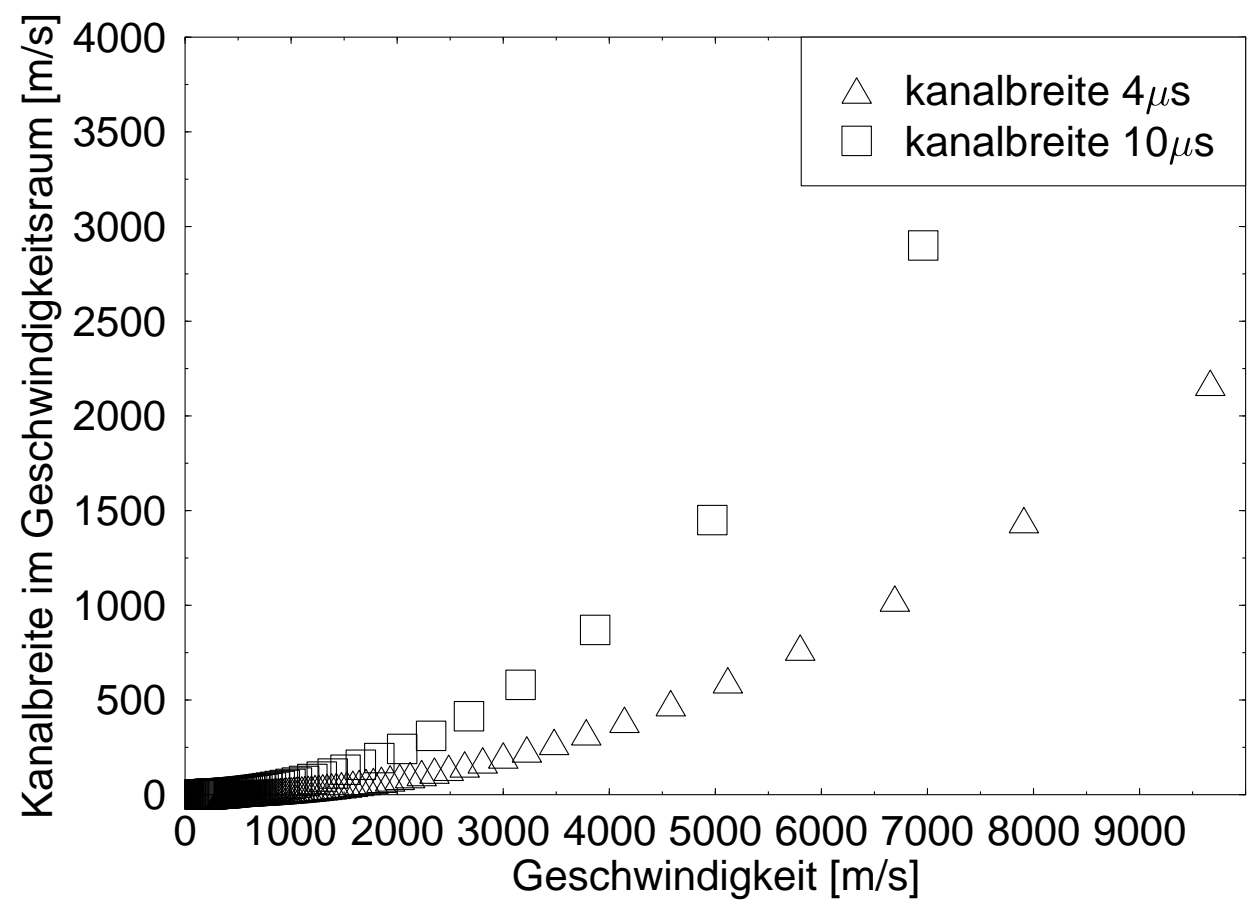

Abb. 4.8: Kanalbreite im Geschwindigkeitsraum in Abhängigkeit von der Geschwindigkeit.

ursachte Informationsverlust hinnehmbar ist; dies ist dann der Fall, wenn das Auflösungsvermögen des TOF-Systems genauso gut oder besser ist als das des Streuexperiments. Die durch die Strahldaten vorgegebenen Breiten der Flugzeitverteilungen liegen je nach System und Stoßenergie bei $200 \frac{\mathrm{m}}{\mathrm{s}}$ bis $700 \frac{\mathrm{m}}{\mathrm{s}}$. Damit wird das TOF-System ab etwa $3000 \frac{\mathrm{m}}{\mathrm{s}}(4 \mu \mathrm{s})$ bzw $2000 \frac{\mathrm{m}}{\mathrm{s}}$ (10 $\mathrm{\mu s}$ Kanalbreite) dominierend (Abb. 4.8). Daraus ergibt sich jedoch keine klare Abgrenzung, denn einerseits sind für die Anpassung der Flugzeitverteilungen immer möglichst viele Punkte zur Bestimmung des Kurvenverlaufs notwendig, andererseits wirkt sich die Messung mit schlechterer Auflösung nur wenig auf die Bestimmung des jeweiligen totalen differentiellen Querschnitts aus. Das gesagte wird illustriert durch den Vergleich von Messungen mit verschiedenen Kanalbreiten (Abb. 4.9).

Insgesamt ergibt sich folgendes Bild:

- Für die Bewertung, ob bei nichtreaktiver Streuung von $\mathrm{Na}$ an $\mathrm{SF}_{6}$ und $\mathrm{SiF}_{4}$ im Grundzustand inelastisches Verhalten zu beobachten ist, waren Messungen mit der minimalen Kanalbreite unabdingbar.

- Da bei allen Messungen der Streuung im Grundzustand ein gutes Signal/RauschVerhältnis erzielt wird, können diese problemlos mit geringer Kanalbreite durchgeführt werden. Daher wurden diese Messungen alle mit $4 \mu s$ Kanalbreite durchgeführt. 
- Die Differenzmessungen mit Laseranregung zeigen grundsätzlich ein schlechtes Signal/RauschVerhältnis und wurden mit einer Kanalbreite von 10 $\mu$ s aufgenommen, um die Meßzeit in akzeptablen Grenzen zu halten. 

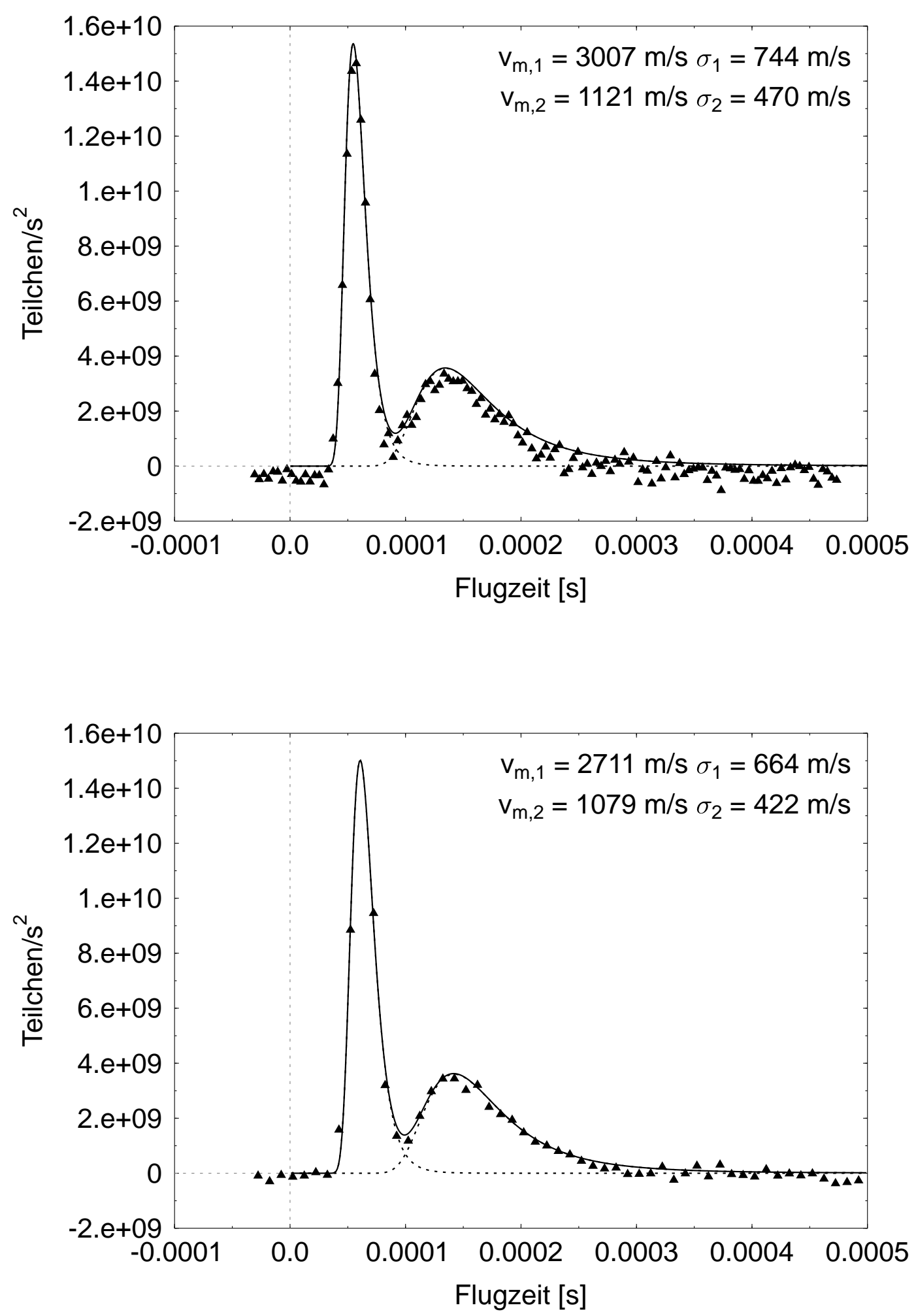

Abb. 4.9: Flugzeitspektren für $\mathrm{Na}^{-\mathrm{SF}_{6}}$ (im Vorgriff auf Kapitel 5). Das obere Spektrum ist mit einer Kanalbreite von $4 \mu s$, das untere mit einer Kanalbreite von $10 \mu s$ in $1 / 4$ der Meßzeit aufgenommen worden. Die integrierten Intensitäten betragen für den elastischen (schnellen) Anteil $20.8 \mathrm{kHz}$ bzw. $22.3 \mathrm{kHz}$ (oben/unten) und für den reaktiven Anteil $19.9 \mathrm{kHz}$ bzw. $19.9 \mathrm{kHz}$. 


\section{Kapitel 5}

\section{Streuung von $\mathrm{SF}_{6}$ an $\mathrm{Na}(3 \mathrm{~S})$ und $\mathrm{Na}(3 \mathrm{P})$}

\section{1 Überblick}

Über das reaktive Streusystem $\mathrm{Na}-S F_{6}$ ist, im wesentlichen aus vorher an der hier verwendeten Apparatur erzielten Ergebnissen [44, 55, 79, 80, 17, 18, 14], einiges bekannt. Daher soll zunächst eine Zusammenfassung bisheriger Erkenntnisse erfolgen, um dann die spezifischen, in dieser Arbeit behandelten Fragestellungen abzugrenzen. Weiterhin wird bei der Darstellung der experimentellen Bedingungen auf die Unterschiede im Aufbau zu den oben zitierten Arbeiten eingegangen.

Bei der Streuung von Natrium an $\mathrm{SF}_{6}$ wird Natriumfluorid entsprechend der Reaktionsgleichung

$$
\mathrm{Na}+\mathrm{SF}_{6} \rightarrow \mathrm{NaF}+\mathrm{SF}_{5}
$$

gebildet; die Reaktion ist exotherm mit einer Exothermizität von $1 \mathrm{eV}$ und einer Reaktionsschwelle von $250 \mathrm{meV}$. Experimentelles Interesse an dieser Reaktion besteht vor allem, weil gute experimentelle Methoden zur Beeinflussung der Reaktanden zur Verfügung stehen. Für das Natrium gibt es die Möglichkeit der elektronischen Anregung, das $\mathrm{SF}_{6}$ besitzt sehr intensive Absorbtionsfrequenzen, die sich mit $\mathrm{CO}_{2}$-Laser-Linien in Koinzidenz befinden.

Vorangegangene Messungen [55] haben ergeben, daß die elektronische Anregung des Natriums nur in der Nähe der Reaktionsschwelle zu einer merklichen Steigerung des totalen Reaktionsquerschnittes führt, während die - energieärmere - Vibrationsanregung des $\mathrm{SF}_{6}$ den totalen Reaktionsquerschnitt um ca. $10 \%$ steigert. Unter der Voraussetzung einer direkten Reaktion wurden totale Reaktionsquerschnitte bestimmt; diese liegen in der Nähe der Reaktionsschwelle bei $26 \AA^{2}$ und dann unabhängig von der Stoßenergie bei etwa $50 \AA^{2}$.

\subsection{Untersuchte Fragestellungen}

\section{Reaktionstyp}

Aus den bisherigen Untersuchungen ist nicht vollständig geklärt, ob bei der Reaktion im Übergangszustand ein zumindest kurzlebiger Stoßkomplex gebildet wird. Der ,weiche“ Charakter des $S F_{6}$ mit seinen vielen, zum Teil energetisch niedrig liegenden Vibrations-Rotations- 
Niveaus sollte eine schnelle Aufnahme der Stoßenergie ermöglichen und damit einen Komplex stabilisieren. Andererseits ist denkbar, daß das $S F_{5}$ lediglich als ,Zuschauer" einer Abstreifreaktion fungiert; dieser Mechanismus würde zu einer schnellen Reaktion führen.

Untersuchungen reaktiver Streuung von Kalium, Rubidium und Cäsium [68] mit $S F_{6}$ zeigen eindeutig die Bildung eines langlebigen Komplexes für die schweren Alkalien Rubidium und Cäsium. Für K-SF 6 , das dem hier untersuchten System am nächsten liegt, ist die Aussage weniger eindeutig, führt aber dennoch auf einen langlebigen Komplex hin. Für das System $\mathrm{Li}-\mathrm{SF}_{6}$ hingegen wurde sowohl das Ergebnis eines langlebigen Komplexes [6] als auch das einer direkten Reaktion [40] angegeben.

Aus den hier gemessenen doppelt differentiellen Querschnitten ergeben sich zwei Möglichkeiten, Informationen über den Reaktionstyp zu gewinnen:

(a). Die Winkelverteilung im Schwerpunktsystem zeigt Charakteristika für bestimmte Reaktionstypen. Reaktionen, für die ein Stoßkomplex gebildet wird, dessen Lebensdauer die Dauer einer Rotation desselben deutlich überschreitet, zeigen eine um $\Theta_{C M}=90^{\circ}$ symmetrische Verteilung, normalerweise bestehend aus einem Vorwärts- und einem Rückwärtsmaximum, begleitet von einem konstanten Querschnitt im Bereich dazwischen. Für Abstreifreaktionen mit großem Querschnitt zeigt sich, abhängig von der Stoßenergie, Vorwärts- oder Seitwärtsstreuung. Direkte Reaktionen mit kleinem Querschnitt hingegen neigen zu Rückwärtsstreuung.

(b). Die Geschwindigkeitsverteilung der Produkte gibt ebenfalls Hinweise bezüglich der Existenz eines Stoßkomplexes [58]. In einem langlebigen Komplex ist Vibrations- und Rotationsrelaxation möglich. Für den Fall vollständiger Relaxation lassen sich statistische Vorhersagen über die Energieverteilung in Rotation, Vibration und Translation der Produkte berechnen. Für eine direkte Reaktion hingegen ist die Wechselwirkungszeit geringer; entsprechend wird ein geringerer Anteil der verfügbaren Energie in die Vibrations-/Rotationsfreiheitsgrade übertragen. Die Reaktionsprodukte weisen dann eine größere kinetische Energie auf.

Es ist evident, daß zur Beurteilung von (a) die Messung der Produkte über den gesamten Winkelbereich $\left(\Theta_{C M}=0^{\circ} . .180^{\circ}\right)$ erfolgen muß. Punkt (b) gibt mehr ein qualitatives Kriterium, da statistisch keine Aussagen darüber gemacht werden können, wie sich eine direkte Reaktion, bei der keine vollständige Relaxation auftritt, verhält. Es ist jedoch aus Experimenten bekannt, daß mit direkten Reaktionen eine geringere Besetzung der Vibrations/Rotationsniveaus einhergeht als statistisch vorhergesagt [48].

Für den Vergleich der Systeme $\mathrm{Na}(3 \mathrm{~S})-\mathrm{SF}_{6}$ und $\mathrm{Na}(3 \mathrm{P})-\mathrm{SF}_{6}$ stellt sich weiterhin die Frage, ob die elektronische Anregung Einfluß auf den Charakter der Reaktion hat. Schließlich gilt zumindest für den Beginn des Stoßprozesses eine andere Potentialfläche, die im Falle von $\mathrm{Na}(3 \mathrm{P})-\mathrm{SF}_{6}$ für eine kollineare Konfiguration (s. Abschnitt 5.5, [14]) einen verhältnismäßig tiefen Potentialtopf aufweist.

\section{Inelastische Prozesse}

Für die Streuung von $\mathrm{Na}$ an $\mathrm{SF}_{6}$ wird auch für große Ablenkwinkel nichtreaktiv gestreutes Natrium beobachtet. Für diesen Anteil stellt sich die Frage, ob signifikant inelastische 
Prozesse vorliegen. Aufgrund der kinematischen Verhältnisse - die Stoßpartner haben ein recht verschiedenes Gewicht - ist kein besonders großer Energieübertrag zu erwarten. Für ein vergleichbares, nichtreaktives System $\left(\mathrm{Ar}_{-} \mathrm{SF}_{6}\right.$, [22]) ist allerdings für den hier verwendeten vergleichbare Stoßenergien nachgewiesen worden, daß inelastische Prozesse mit einem Energieübertrag von bis zu $40 \%$ der verfügbaren Energie vorliegen. Dies hat nicht notwendigerweise zur Folge, daß auch für Na-SF 6 inelastische Prozesse eine Rolle spielen. Es ist ebensogut denkbar, daß diejenigen Stöße, deren Stoßparameter klein genug ist, Vibrations/Rotationsanregung auszulösen, zur Reaktion führen.

Die „Schlüsselqualifikation“ des experimentellen Aufbaus ist hier das Auflösungsvermögen bezüglich der Geschwindigkeit der gestreuten Teilchen (s. Abschnitt 4.3.4) und die Kenntnis der für reine elastische Streuung zu erwartenden Geschwindigkeiten, um inelastische und elastische Prozesse unterscheiden zu können.

Eine besondere Form inelastischer Streuung ist für das System $\mathrm{Na}(3 \mathrm{P})-\mathrm{SF}_{6}$ möglich: Die Abregung zu Na(3S) im Stoß bei Umwandlung der Anregungsenergie des Natriums in innere Energie des $\mathrm{SF}_{6}$ und Translationsenergie der Stoßpartner. Ein solcher Löschprozess liegt für das nichtreaktive System Na-SiF 4 vor und wird in Kap. 6 ausführlich beschrieben.

Für die Identifikation von nichtreaktiv gestreutem Natrium, das aus einem solchen Löschprozess stammt, ist festzustellen, ob für entsprechend vorhergesagte Geschwindigkeiten Anteile nichtreaktiv gestreuten Natriums gemessen werden können. Für die Vorhersage wird davon ausgegangen, daß der Prozeß - wenn überhaupt - analog dem System $\mathrm{Na}^{-\mathrm{SiF}_{4}}$ abläuft.

\subsection{Experimentelle Bedingungen}

Der experimentelle Aufbau ist im Kap. 3 im Detail beschrieben; es soll nur noch auf einige spezielle Änderungen bzw. Unterschiede zu den vorangegangenen Arbeiten eingegangen werden. Für die hier dargestellten Experimente an $\mathrm{Na}(3 \mathrm{~S})-\mathrm{SF}_{6}$ und $\mathrm{Na}(3 \mathrm{P})-\mathrm{SF}_{6}$ wurde als Molekularstrahlquelle für das $\mathrm{SF}_{6}$ ein Düsenstrahl eingesetzt; in [55] wurde für einige Experimente eine Vielkanaldusche verwendet.

Um auch für das System $\mathrm{Na}(3 \mathrm{P})-\mathrm{SF}_{6}$ das Produkt $\mathrm{NaF}$ vom nichtreaktiv gestreuten Natrium sicher trennen zu können, wurde ein Zwei-Farben Anregungs- und Detektionsschema im LIF-Detektor eingeführt; die Beschreibung findet sich in 4.1.3. Durch die Zwei-FarbenAnregung eine erheblich gesteigerte Anregungseffizienz erreicht. Aus den Flugzeitspektren von $\mathrm{Na}(3 \mathrm{P})-\mathrm{SF}_{6}$ wurde auch die experimentelle Anregungseffizienz zu $27 \pm 4 \%$ bestimmt. Das Verfahren ist ebenfalls in 4.1 .3 beschrieben.

Der zugängliche Laborwinkelbereich konnte soweit gesteigert werden, daß mit dem LangmuirTaylor-Detektor $\Theta_{L A B}=140^{\circ}$ erreicht werden kann. Damit kann für die reaktive Streuung im Grundzustand und bei Stoßenergien unter ca. $1 \mathrm{eV}$ auch für die reaktive Streuung im angeregten Zustand bis $\Theta_{C M}=180^{\circ}$ gemessen werden. Um die Messung auf diesen Winkeln auch zu ermöglichen, war eine Verbesserung des Signal/Rausch-Verhältnisses notwendig. Hierzu tragen bei in erster Linie die größere Anregungseffizienz durch die Zwei-FarbenAnregung für die Messungen mit $\mathrm{Na}(3 \mathrm{P})$ und weiterhin eine großflächige Abschirmung des Primärstrahlchoppers mit einem $\mathrm{N}_{2}$ (fl.)-gekühlten Kupferblech für Messungen im Grundzustand.

Damit Messungen der Geschwindigkeit des elastischen bzw. inelastischen Anteils nicht 
durch das Auflösungsvermögen des TOF-Systems beeinträchtigt werden, wurde die Maximaldrehzahl auf $1 \mathrm{kHz}$ erhöht und damit die Kanalbreite auf $4 \mu$ s reduziert.

Die beiden dargestellten Messungen wurden unter folgenden Bedingungen ausgeführt :

(a). Der Primärstrahl hatte eine Geschwindigkeit von $4400 \frac{\mathrm{m}}{\mathrm{s}}$ bei einer Halbwertsbreite von $380 \frac{\mathrm{m}}{\mathrm{s}}$; als Seedgas kam Wasserstoff zum Einsatz. Die Sekundärdüse wurde bei einer Temperatur von $298 \mathrm{~K}$ betrieben. Aus [55] wird daraus eine Geschwindigkeit von $410 \frac{\mathrm{m}}{\mathrm{s}}$ entnommen. Es ergibt sich eine mittlere Stoßenergie von $2.0 \mathrm{eV}$ im Schwerpunktsystem.

(b). Der Primärstrahl hatte eine Geschwindigkeit von $2374 \frac{\mathrm{m}}{\mathrm{s}}$ bei einer Halbwertsbreite von $280 \frac{\mathrm{m}}{\mathrm{s}}$; als Seedgas kam Wasserstoff zum Einsatz. Die Sekundärdüse wurde ebenfalls bei einer Temperatur von $298 \mathrm{~K}$ betrieben. Es ergibt sich eine mittlere Stoßenergie von $0.60 \mathrm{eV}$ im Schwerpunktsystem.

\subsection{Experimentelle Ergebnisse}

\section{Flugzeitspektren}

Wie bereits in Kap. 3 gesagt, erfolgt die Unterscheidung zwischen nichtreaktiv gestreutem Natrium und natriumhaltigen Verbindungen durch die Anwendung verschiedener Detektoren, eines LIF-(laser-induced-fluorescence)- und eines LT-(Langmuir-Taylor)-Detektors. Für die hier erzielten Ergebnisse wurde der LIF-Detektor nur eingesetzt, um zu verifizieren, daß nur der schnelle, elastische bzw. inelastische Anteil nichtreaktiv gestreutes Natrium enthält (Dieser Anteil wird im folgenden analog zu [47] ,pseudoelastisch“ genannt). Aufgrund der Zwei-Linien-Anregung im Detektor konnten derartige Messungen auch für $\mathrm{Na}(3 \mathrm{P})-\mathrm{SF}_{6}$ ausgeführt werden. Auch diese Messungen ergeben, daß sich nur im pseudoelastischen Anteil der Differenzspektren nichtreaktiv gestreute Teilchen befinden. Ein Löschprozess

$$
N a(3 P)+S F_{6}\left(v=v_{0}\right) \rightarrow N a(3 S)+S F_{6}\left(v=v^{\prime}\right)
$$

würde in Analogie zu den für $\mathrm{Na}(3 \mathrm{P})-\mathrm{SiF}_{4}$ (Kap. 6) erzielten Resultaten eindeutig beobachtbar sein. Aus der Nicht-Existenz eines entsprechenden langsamen, nichtreaktiven Anteils kann geschlossen werden, daß für $\mathrm{Na}(3 \mathrm{P})-\mathrm{SF}_{6}$ kein Löschprozess auftritt.

Für den pseudoelastischen Anteil der Streuung im angeregten Zustand findet sich kein Hinweis darauf, daß sich Änderungen in der Geschwindigkeit oder Breite im Verhältnis zur Streuung im Grundzustand ergeben, abgesehen von einer geringfügigen Verringerung der Breite durch die unterschiedliche Position des Choppers (Auf der Flugstrecke zwischen Chopper und Streuzone laufen die vom Chopper präparierten „Pakete“ etwas auseinander; für die Differenzspektren hingegen findet die Modulation in der Streuzone statt). Allerdings wäre das Auflösungsvermögen auch nicht ausreichend, an dieser Stelle geringe Änderungen aufzuspüren. Im Umkehrschluß wird für die Auswertung vorausgesetzt, daß Position und Breite des pseudoelastischen Anteils im Differenzspektrum durch die entsprechenden Werte des Grundzustandsspektrums richtig wiedergegeben werden. 
Im folgenden sind exemplarisch Flugzeitspektren der bei $0.6 \mathrm{eV}$ durchgeführten Messung abgebildet. Die gestrichelten Linien geben die einzelnen Anteile wieder, die durchgezogene Linie die an das Spektrum angepaßte Summenkurve. Für die Bewertung der Spektren ist zu berücksichtigen, daß die verschiedenen Anteile zu verschiedenen Winkeln im Schwerpunktsystem gehören; die Zuordnung läßt sich aus dem Newton-Diagramm (Abb. 5.7) ablesen.

\section{Auftragung der Meßergebnisse}

Der Gang der Auswertung ist in Abschnitt 4.2.3 beschrieben. Dementsprechend sind die Maxima und Positionen der einzelnen Anteile in den Flugzeitspektren in Newton-Diagramme eingetragen. Es ergeben sich also für jede Stoßenergie drei Newton-Diagramme (für pseudoelastische Streuung, reaktive Streuung im Grundzustand und im angeregten Zustand). In diese Newton-Diagramme zusätzlich eingetragen sind zum Vergleich

- für die nichtreaktive Streuung der Newton-Kreis für ideale, elastische Streuung und

- für reaktive Streuung die der maximal erreichbaren kinetischen Energie entsprechende Geschwindigkeit, die für das System erreichbar wäre, wenn die gesamte verfügbare Energie, also die Stoßenergie, die Exothermizität und für Stöße mit Na(3P) die Anregungsenergie in kinetische Energie umgesetzt würden (äußere durchgezogene Linie) sowie die aufgrund statistischer Überlegungen berechnete a priori-Verteilung, deren wahrscheinlichste Geschwindigkeit mit einer durchgezogenen Linie dargestellt ist. Gestrichelte Linien deuten die Halbwertsbreite an. Die Berechnung ist in Abschnitt 5.5 angegeben.

Des weiteren sind die Winkelverteilungen im Schwerpunktsystem für die jeweiligen Anteile angegeben. Dabei ist die Anregungseffizienz für den Vergleich der Na(3P)- mit den $\mathrm{Na}(3 \mathrm{~S})$-Messungen bereits eingearbeitet, so daß die Winkelverteilungen für Streuung im angeregten und im Grundzustand direkt untereinander verglichen werden können. Für den Vergleich zwischen den Winkelverteilungen der beiden Stoßenergieen ist noch zu berücksichtigen, daß bei der hohen Stoßenergie die sechsfache Primärstrahlintensität vorlag.

Aus den beiden dargestellten Meßreihen lassen sich einige Trends ableiten :

Der pseudoelastische Anteil neigt bei der niedrigen Stoßenergie zu starker Inelastizität schon für kleine Winkel, während bei der hohen Stoßenergie erst kurz vor dem Aussetzen des pseudoelastischen Anteils eine stärkere Inelastizität zu beobachten ist. Eine im folgendenden Abschnitt präsentierte, mit einer Stoßenergie von $1.3 \mathrm{eV}$ zwischen den beiden präsentierten Meßreihen liegende Messung entspricht diesem Trend. Die Winkelverteilung für den pseudoelastischen Anteil ist strukturlos und fällt monoton zu größeren Winkeln hin ab. Ab einem Schwerpunktswinkel von ca. $50^{\circ}$ (bei $E_{C M}=2.0 \mathrm{eV}$ ) bzw. ca. $80^{\circ}$ (bei $E_{C M}=0.6 \mathrm{eV}$ ) wird der pseudoelastische Anteil bedeutungslos den jeweiligen reaktiven Anteilen gegenüber.

Der im Grundzustand gemessene, reaktive Anteil zeigt für beide Stoßenergien eine winkelabhängige Geschwindigkeitsverteilung. Die Geschwindigkeiten im Bereich um $\Theta_{C M}=$ $90^{\circ}$ sind niedriger als diejenigen in Vorwärts- und Rückwärtsrichtung. Die Winkelverteilungen enthalten jeweils ein Maximum für $\Theta_{C M}=0^{\circ}$ und für $\Theta_{C M}=180^{\circ}$; das zweite ist aller- 


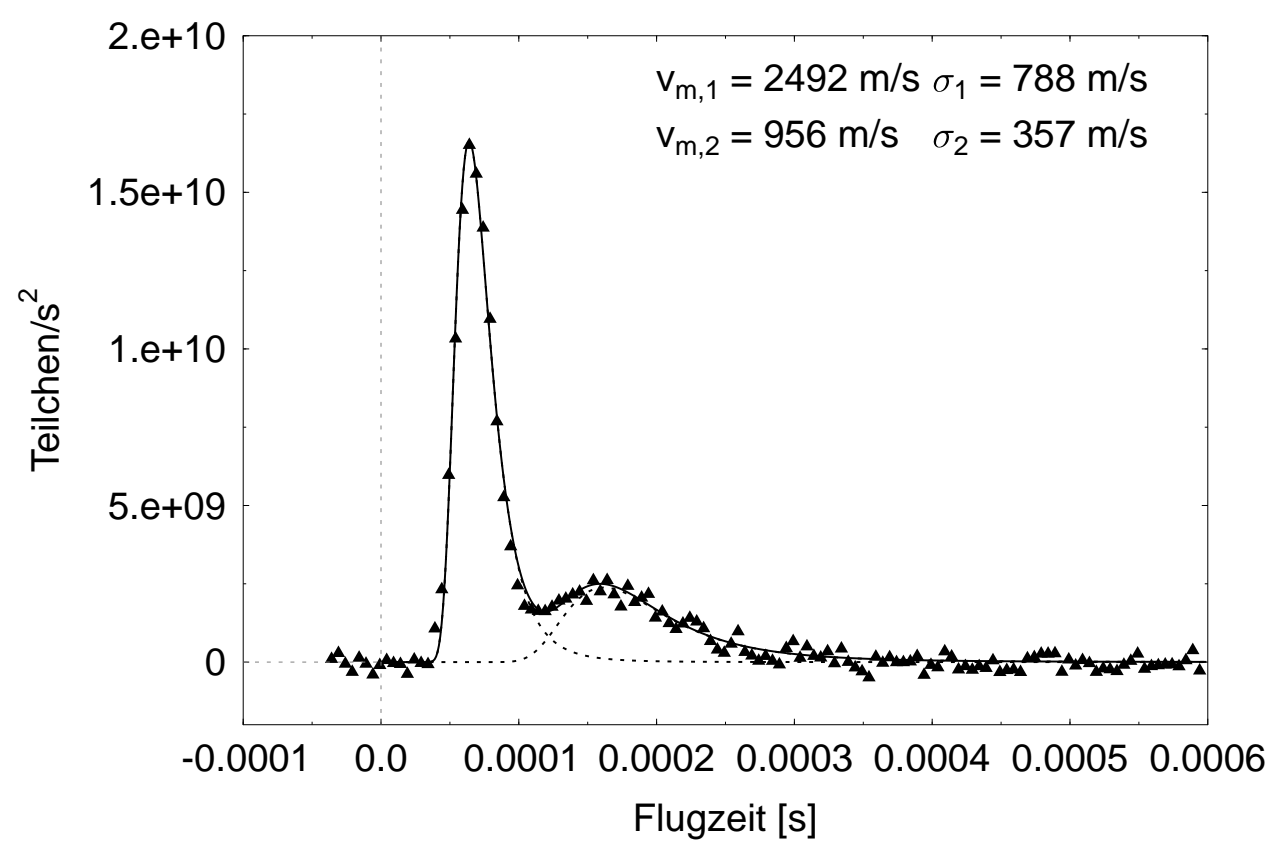

Abb. 5.1: Flugzeitspektrum bei $\Theta_{L A B}=20^{\circ}$ 


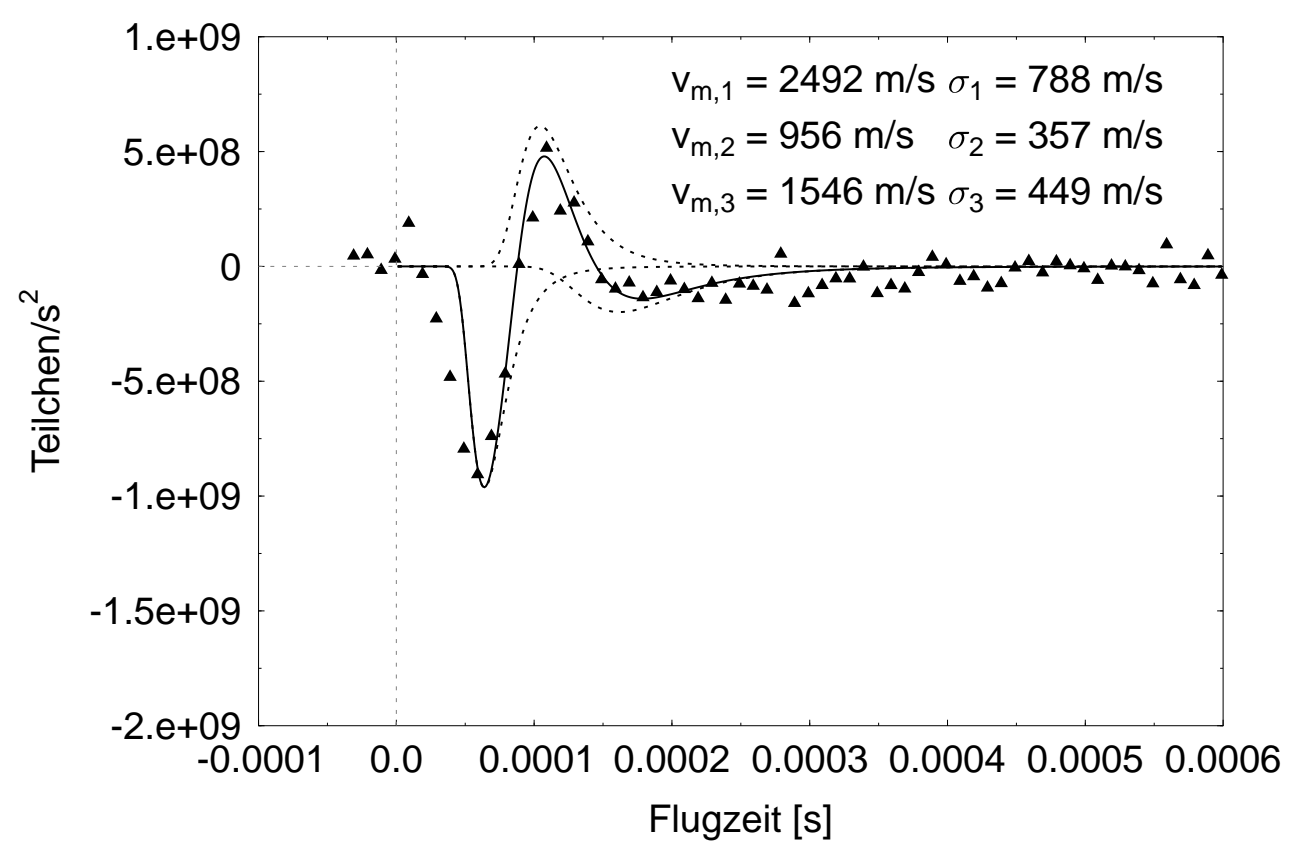

Abb. 5.2: Differenz-Flugzeitspektrum bei $\Theta_{L A B}=20^{\circ}$ 


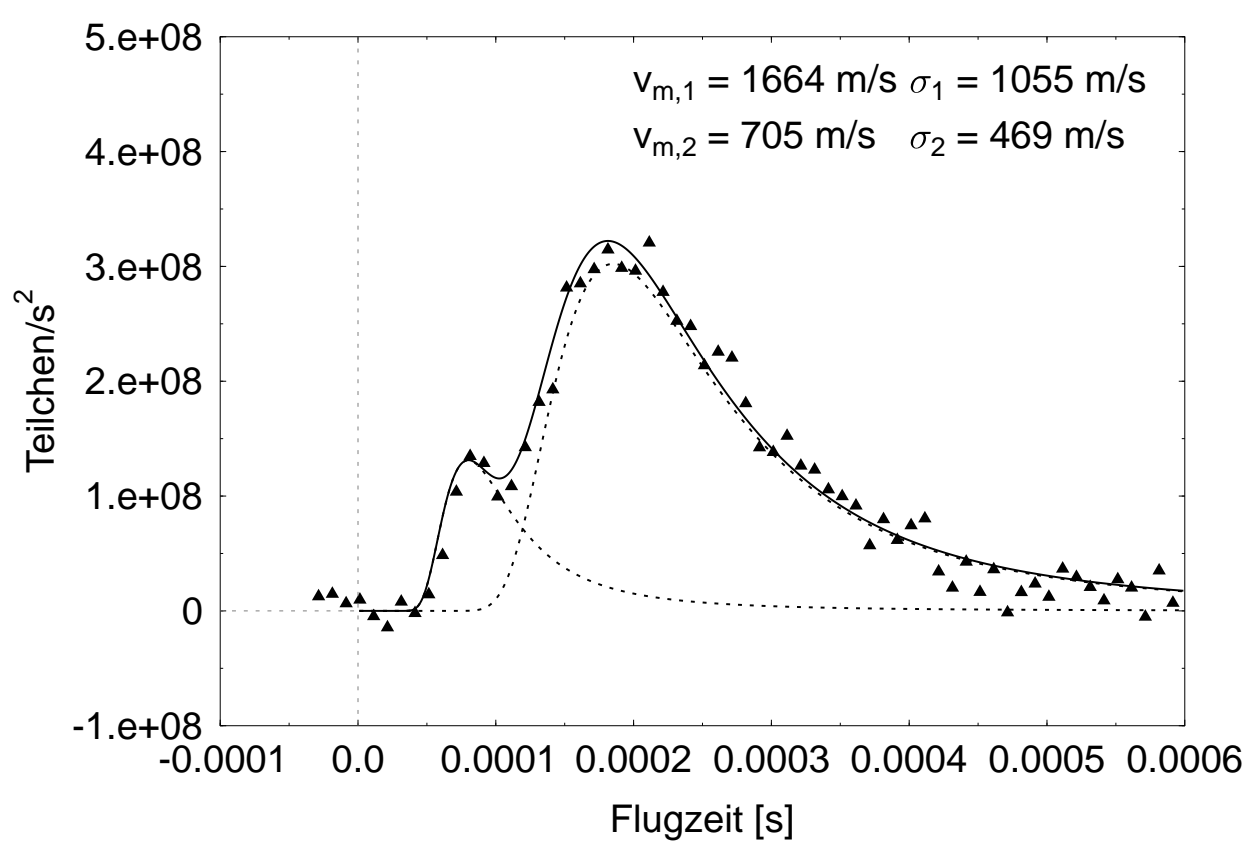

Abb. 5.3: Flugzeitspektrum bei $\Theta_{L A B}=70^{\circ}$ 


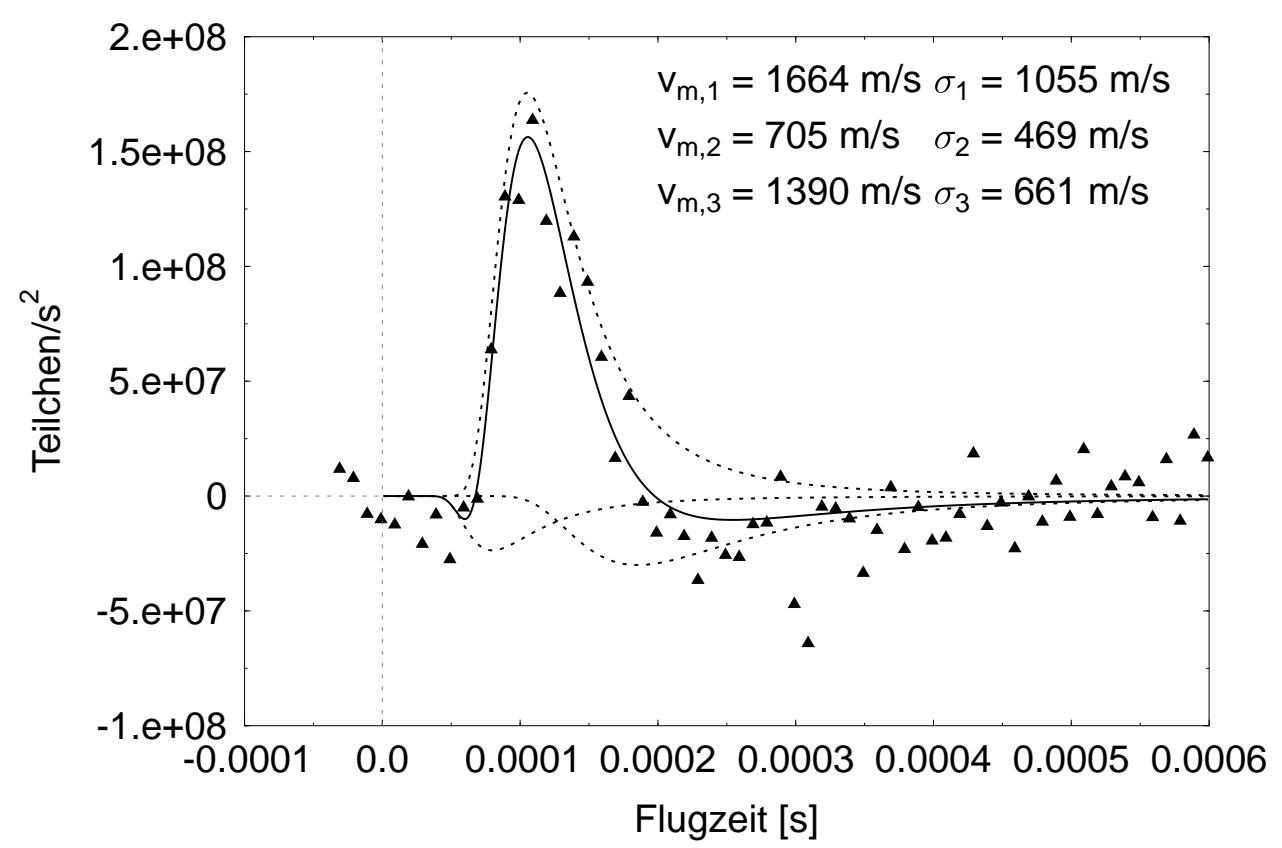

Abb. 5.4: Differenz-Flugzeitspektrum bei $\Theta_{L A B}=70^{\circ}$ 


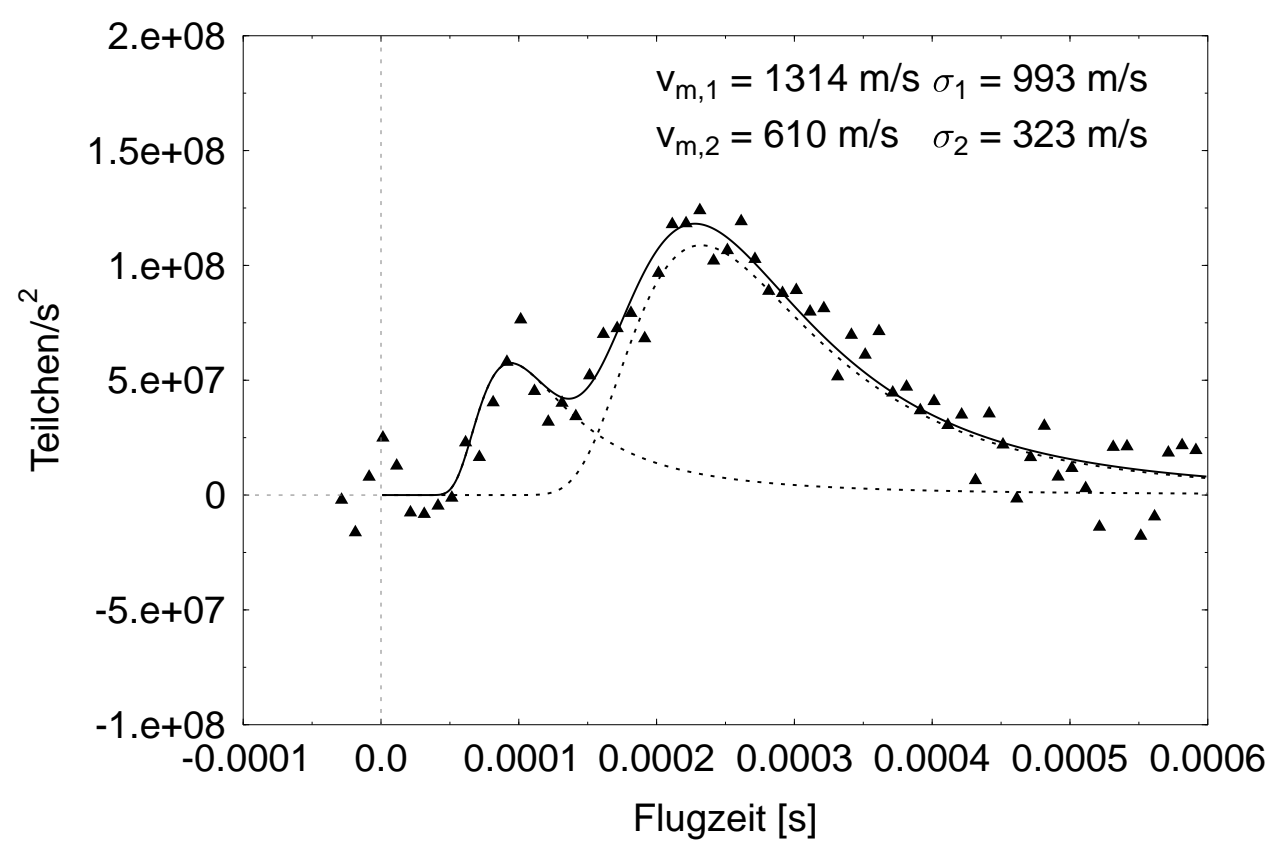

Abb. 5.5: Flugzeitspektrum bei $\Theta_{L A B}=120^{\circ}$ 


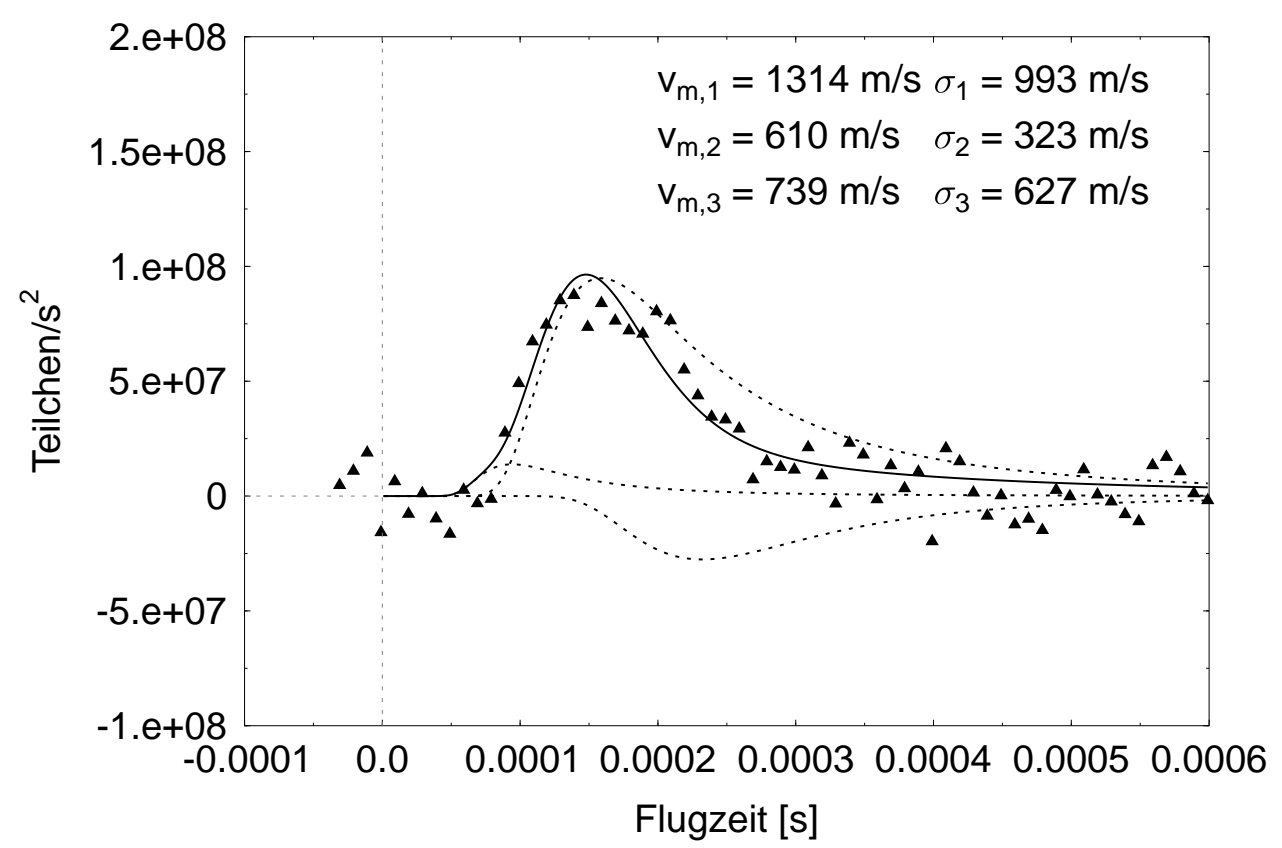

Abb. 5.6: Differenz-Flugzeitspektrum bei $\Theta_{L A B}=120^{\circ}$ 


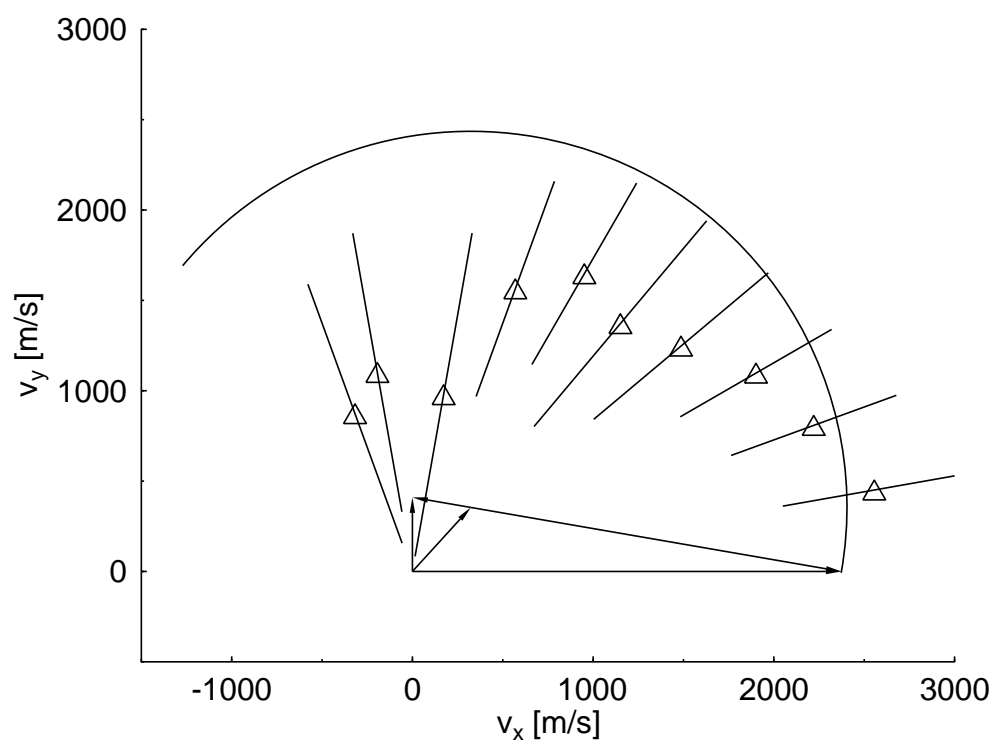

Abb. 5.7: Auftragung des nichtreaktiven Anteils aus der Streuung von $\mathrm{Na}(3 \mathrm{~S})$ und $\mathrm{Na}(3 \mathrm{P})$ an $\mathrm{SF}_{6}$ im Newton-Diagramm. Die zugehörige Stoßenergie ist $0.6 \mathrm{eV}$. 


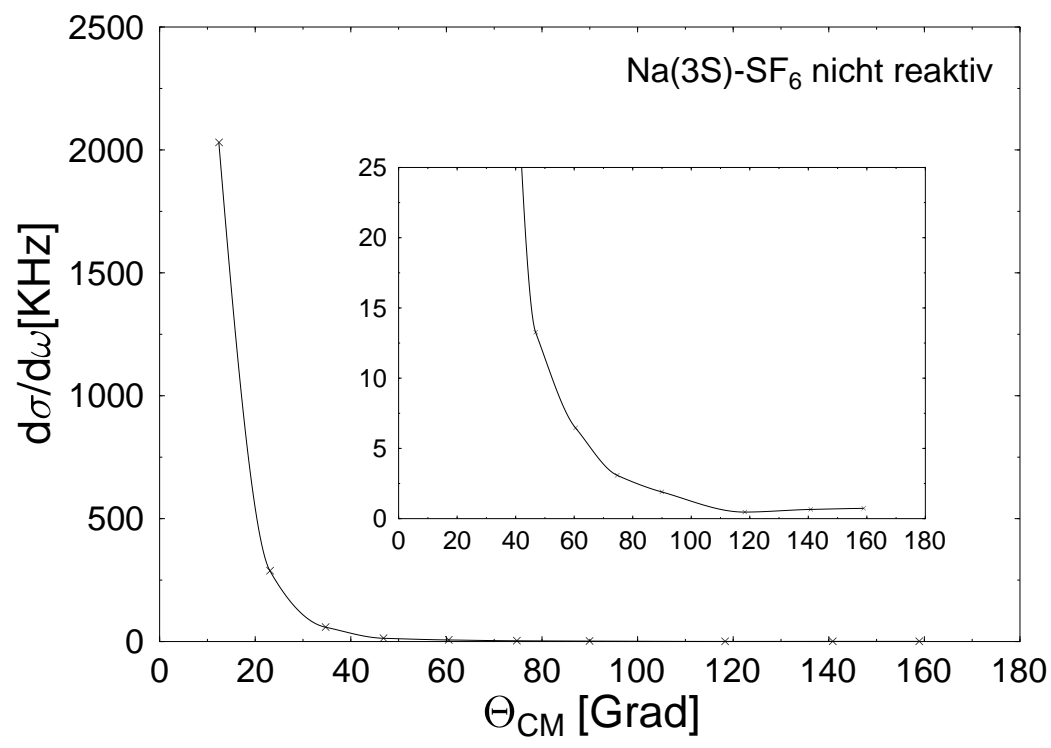

Abb. 5.8: Winkelverteilung des nichtreaktiven Anteils aus der Streuung von $\mathrm{Na}(3 \mathrm{~S})$ an $\mathrm{SF}_{6}$ im Schwerpunktsystem. Die zugehörige Stoßenergie ist $0.6 \mathrm{eV}$. 


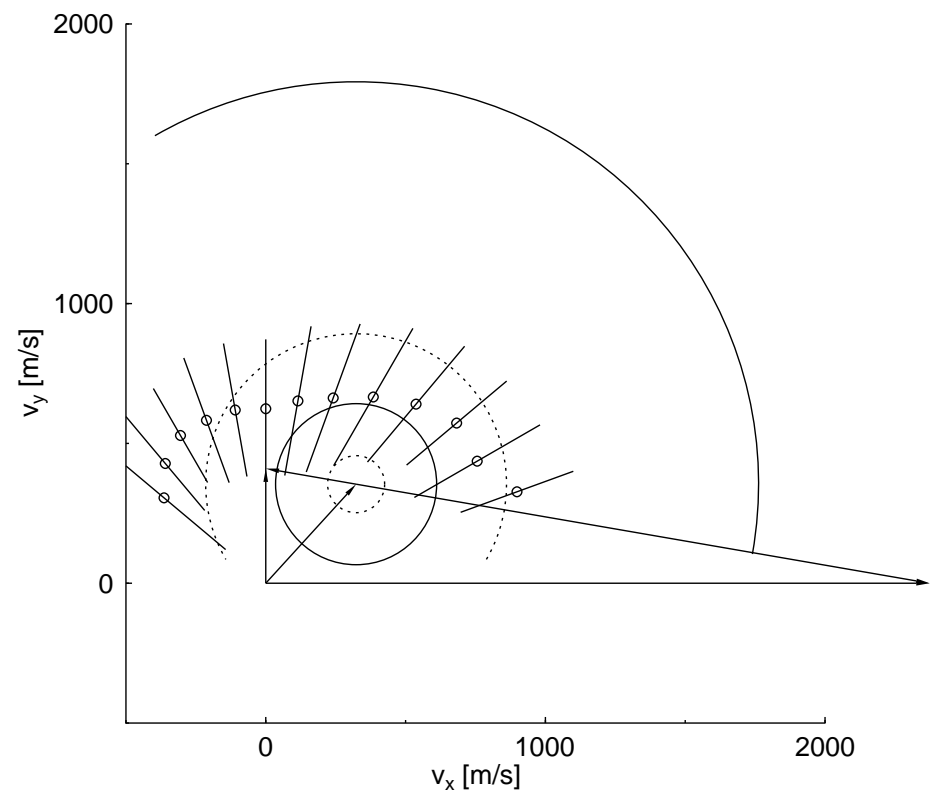

Abb. 5.9: Auftragung des reaktiven Anteils aus der Streuung von $\mathrm{Na}(3 \mathrm{~S})$ an $\mathrm{SF}_{6}$ im NewtonDiagramm. Die zugehörige Stoßenergie ist $0.6 \mathrm{eV}$. 


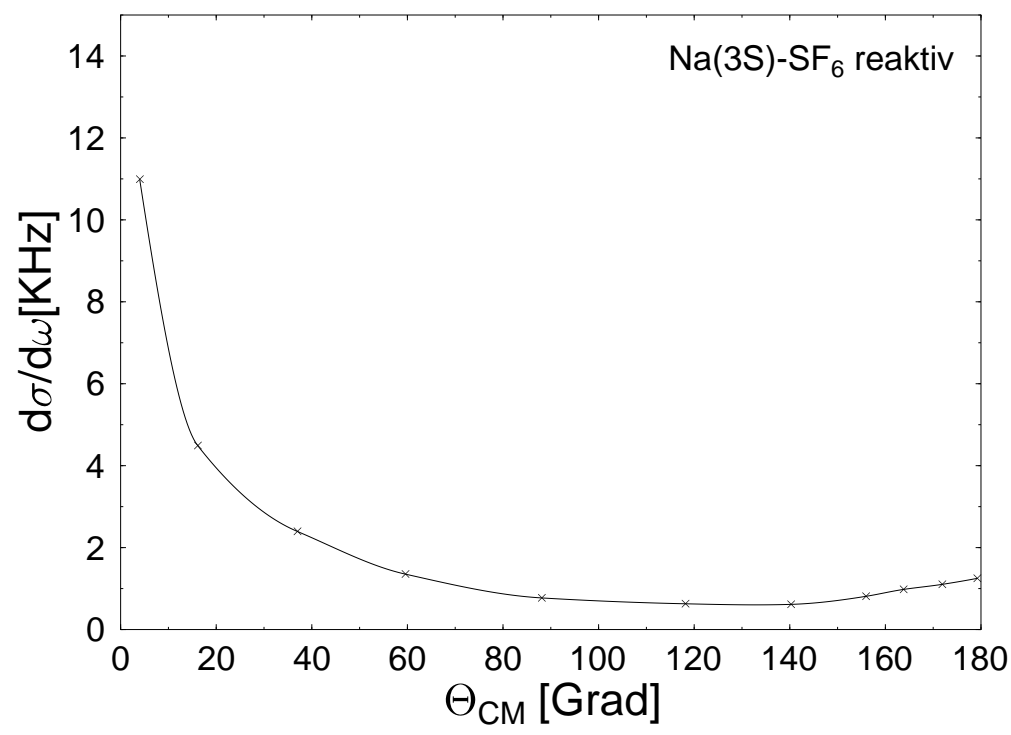

Abb. 5.10: Winkelverteilung des reaktiven Anteils aus der Streuung von $\mathrm{Na}(3 \mathrm{~S})$ an $\mathrm{SF}_{6}$ im Schwerpunktsystem. Die zugehörige Stoßenergie ist $0.6 \mathrm{eV}$. 


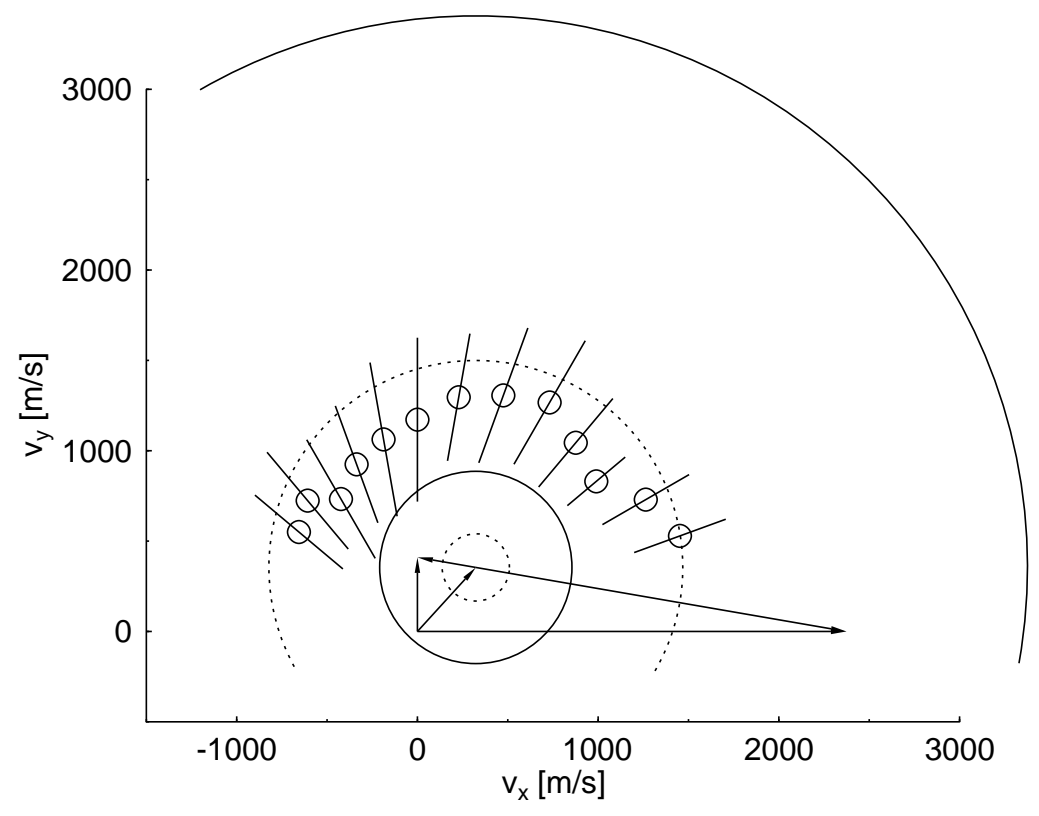

Abb. 5.11: Auftragung des reaktiven Anteils aus der Streuung von $\mathrm{Na}(3 \mathrm{P})$ an $\mathrm{SF}_{6}$ im Newtondiagramm. Die zugehörige Stoßenergie ist $0.6 \mathrm{eV}$. 


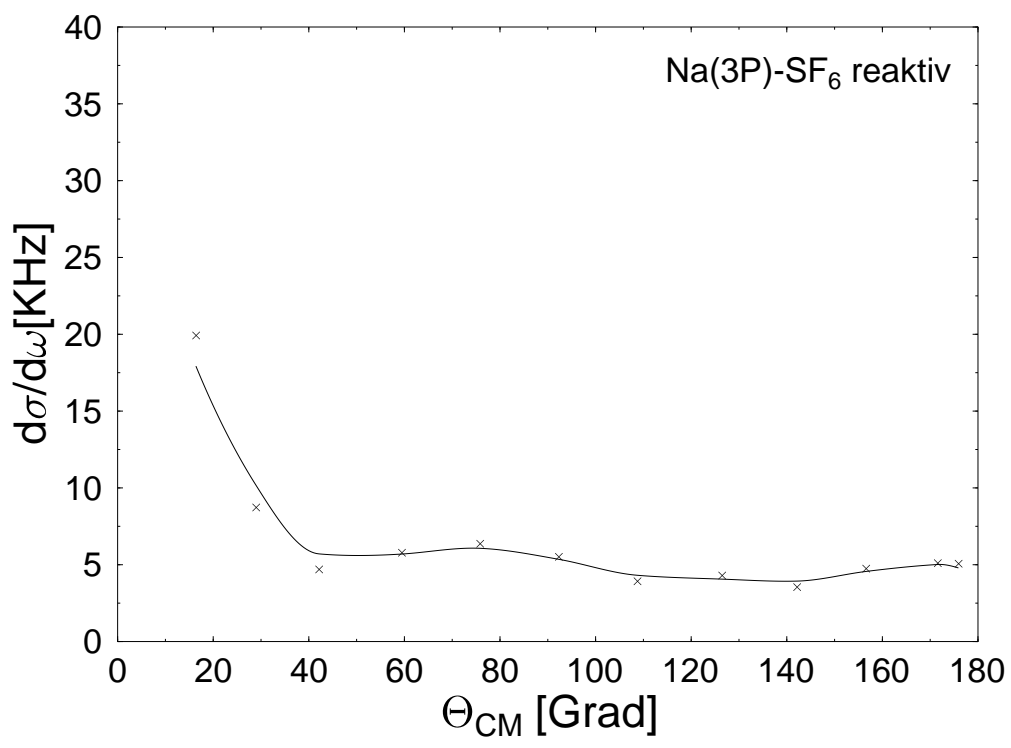

Abb. 5.12: Winkelverteilung des reaktiven Anteils aus der Streuung von $\mathrm{Na}(3 \mathrm{P})$ an $\mathrm{SF}_{6}$ im Schwerpunktsystem. Die zugehörige Stoßenergie ist $0.6 \mathrm{eV}$. 


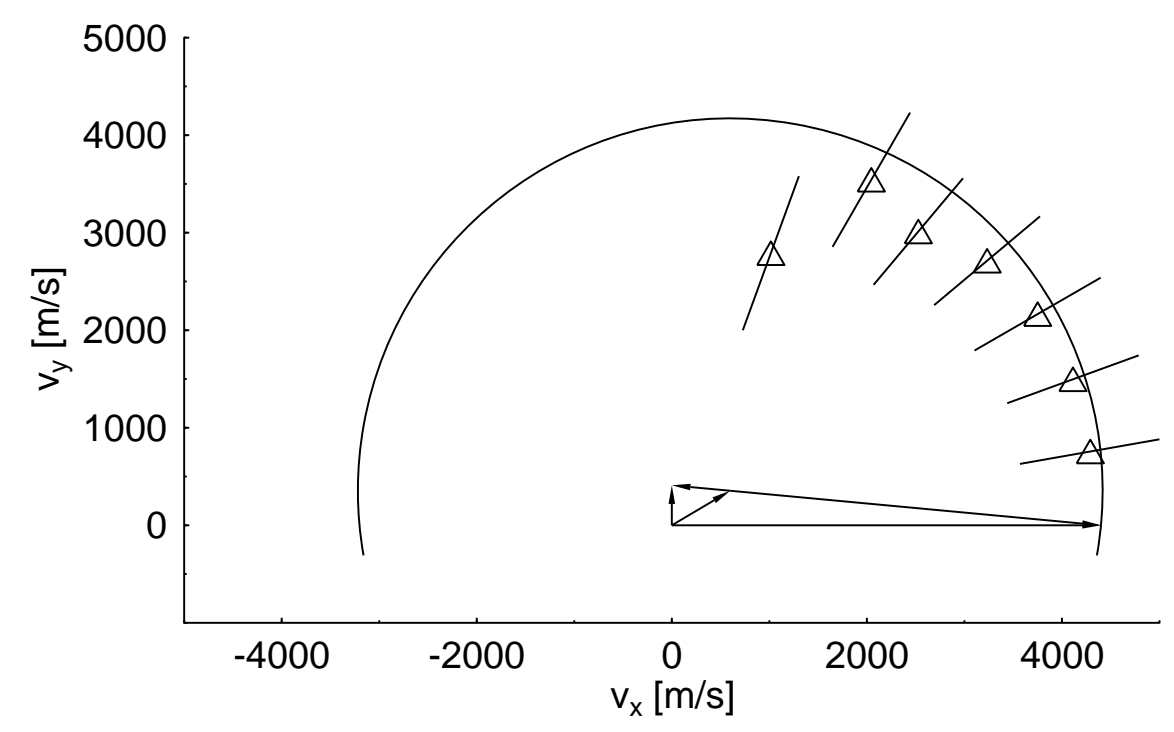

Abb. 5.13: Auftragung des nichtreaktiven Anteils aus der Streuung von $\mathrm{Na}(3 \mathrm{~S})$ und $\mathrm{Na}(3 \mathrm{P})$ an $\mathrm{SF}_{6}$ im Newton-Diagramm. Die zugehörige Stoßenergie ist $2.0 \mathrm{eV}$. 


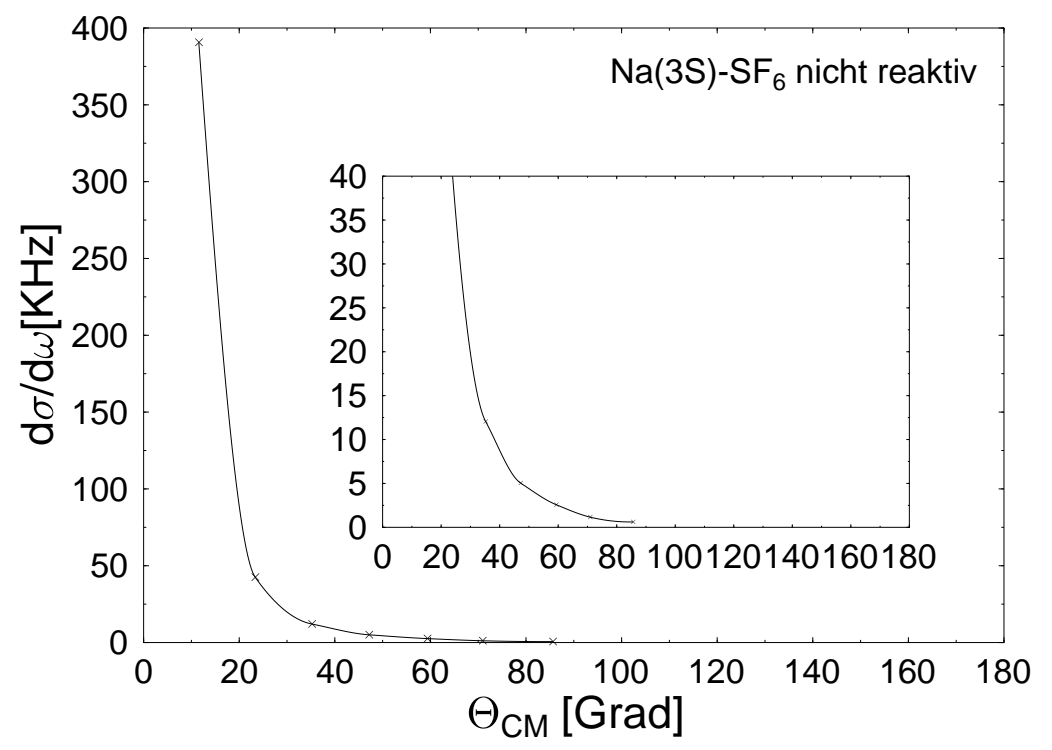

Abb. 5.14: Winkelverteilung des nichtreaktiven Anteils aus der Streuung von $\mathrm{Na}(3 \mathrm{~S})$ an $\mathrm{SF}_{6}$ im Schwerpunktsystem. Die zugehörige Stoßenergie ist $2.0 \mathrm{eV}$. 


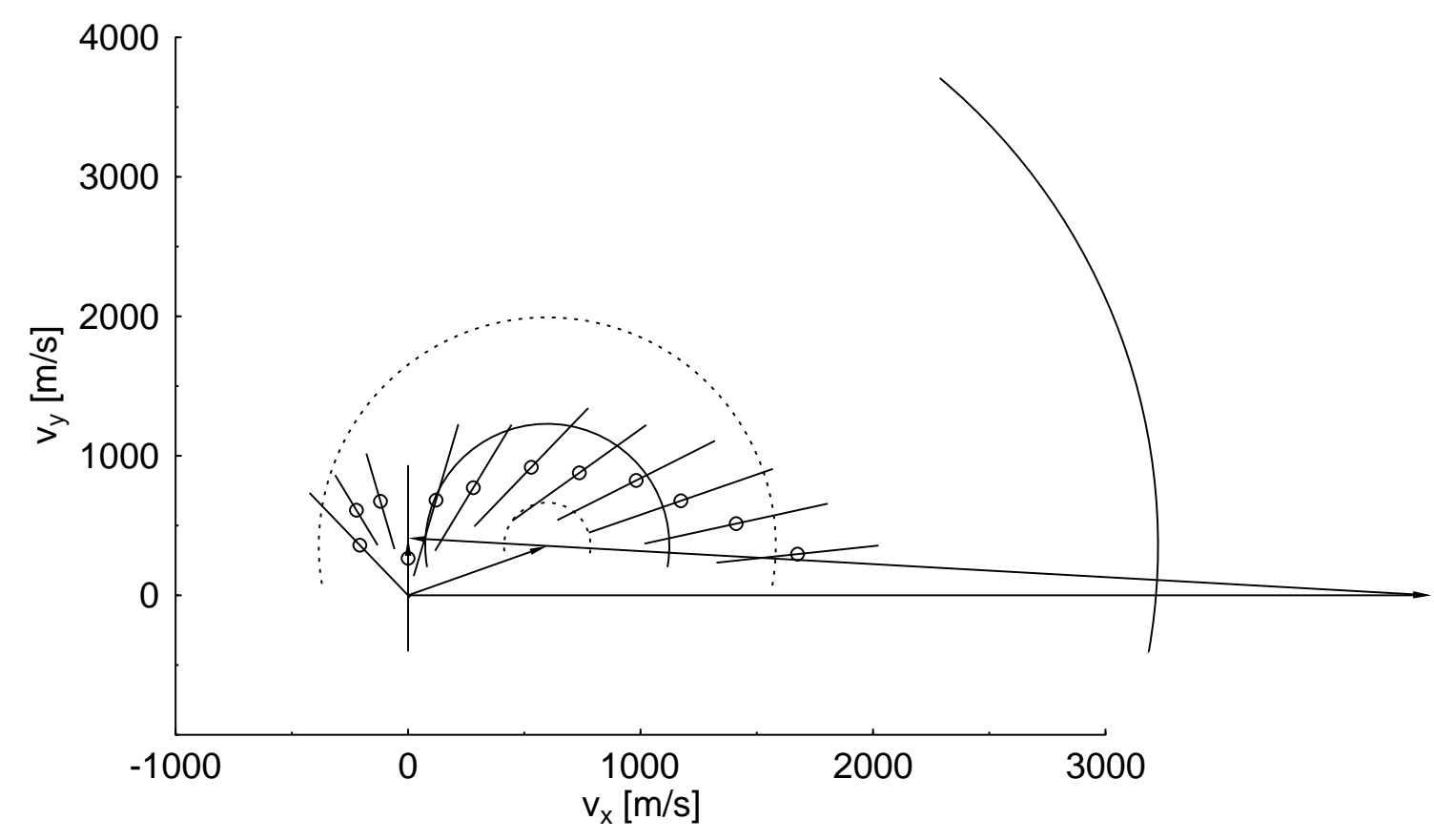

Abb. 5.15: Auftragung des reaktiven Anteils aus der Streuung von $\mathrm{Na}(3 \mathrm{~S})$ an $\mathrm{SF}_{6}$ im NewtonDiagramm. Die zugehörige Stoßenergie ist $2.0 \mathrm{eV}$. Der Einbruch für $\Theta_{L A B}=90^{\circ}$ ist auf Wechselwirkung mit dem Sekundärstrahl zurückzuführen. 


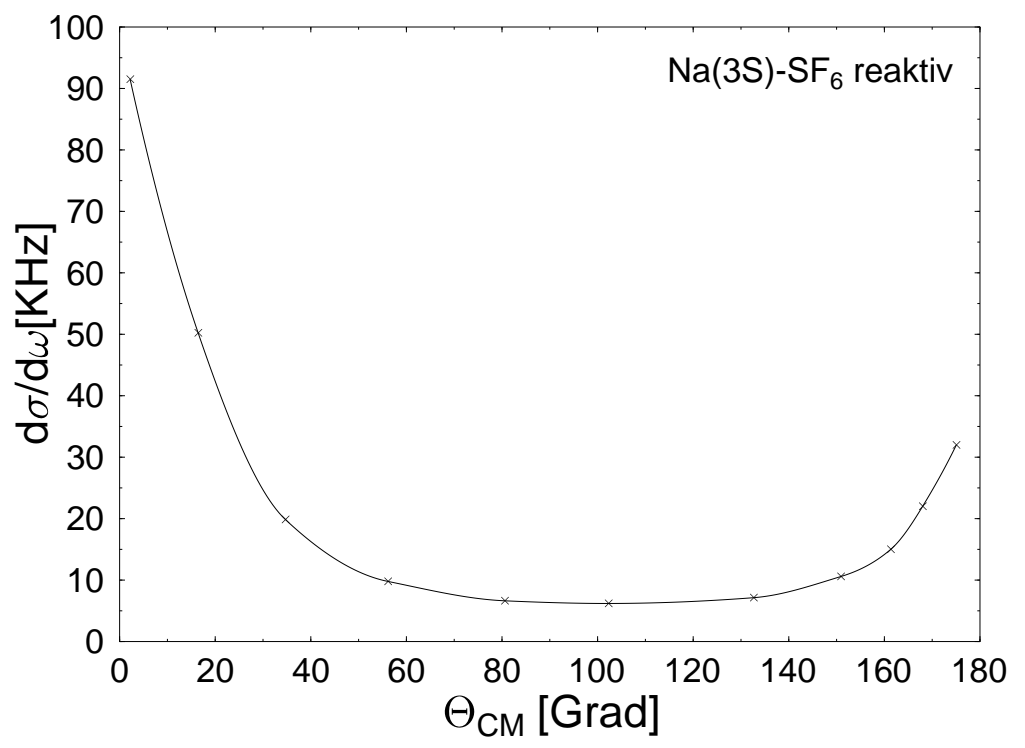

Abb. 5.16: Winkelverteilung des reaktiven Anteils aus der Streuung von $\mathrm{Na}(3 \mathrm{~S})$ an $\mathrm{SF}_{6}$ im Schwerpunktsystem. Die zugehörige Stoßenergie ist $2.0 \mathrm{eV}$. 


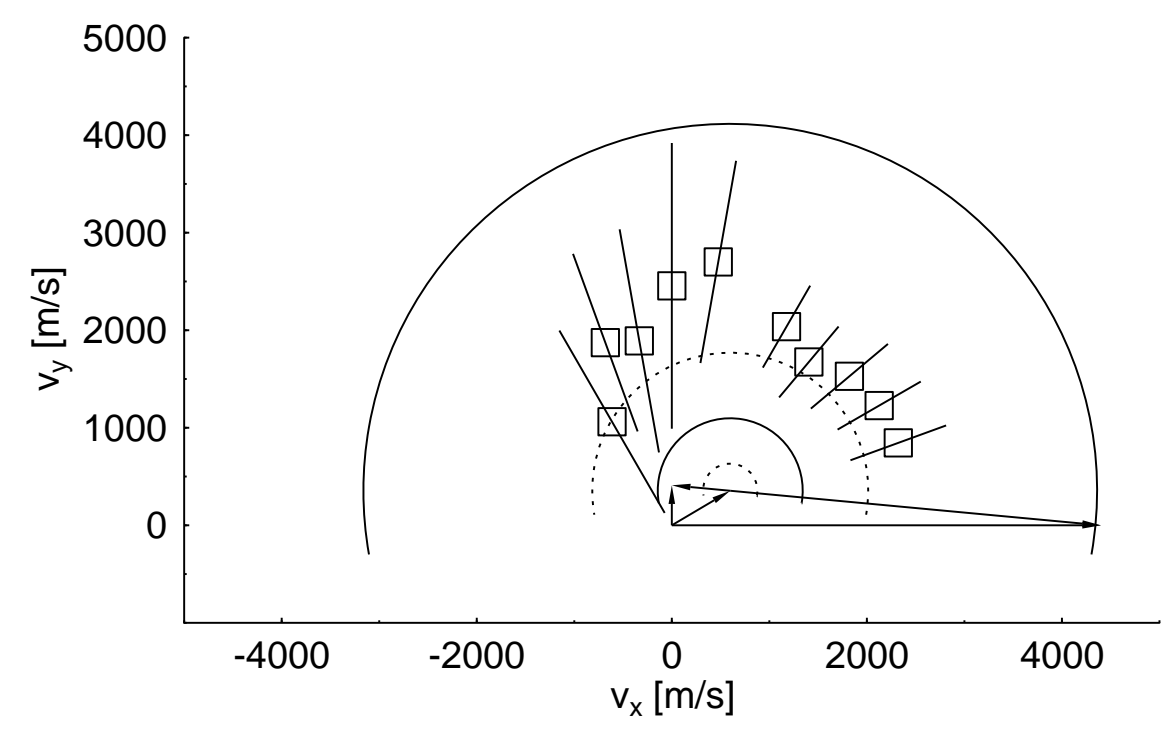

Abb. 5.17: Auftragung des reaktiven Anteils aus der Streuung von $\mathrm{Na}(3 \mathrm{P})$ an $\mathrm{SF}_{6}$ im Newtondiagramm. Die zugehörige Stoßenergie ist $2.0 \mathrm{eV}$. 


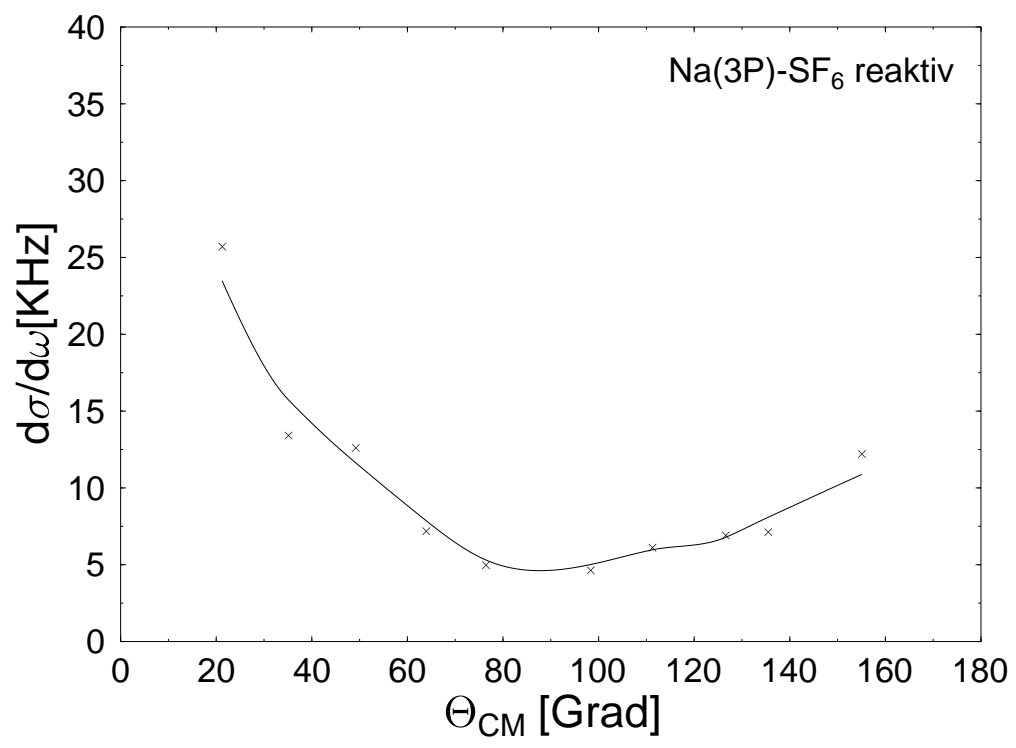

Abb. 5.18: Winkelverteilung des reaktiven Anteils aus der Streuung von $\mathrm{Na}(3 \mathrm{P})$ an $\mathrm{SF}_{6}$ im Schwerpunktsystem. Die zugehörige Stoßenergie ist $2.0 \mathrm{eV}$. 
dings für die niedrige Stoßenergie sehr schwach ausgeprägt und auch bei $2.0 \mathrm{eV}$ wesentlich kleiner als das Vorwärtsmaximum.

Eine ähnliche Situation ergibt sich für die reaktive Streuung im angeregten Zustand. Für die hohe Stoßenergie liegen aufgrund der ungünstigeren kinematischen Bedingungen Werte nur in einem eingeschränkten Winkelbereich vor $\left(\Theta_{C M}=20^{\circ}\right.$ bis $\left.155^{\circ}\right)$, es ist jedoch ein Anstieg zu großen Winkel hin erkennbar. Für die niedrige Stoßenergie zeigt sich im Bereich von $\Theta_{C M}=40^{\circ}$ bis $\Theta_{C M}=180^{\circ}$ ein im wesentlichen konstanter Querschnitt. Im Gegensatz zu Streuung im Grundzustand ist jedoch die Geschwindigkeitsverteilung nicht so stark winkelabhängig.

\subsection{Interpretation der Ergebnisse}

\section{Inelastische Streuung}

Um zu überprüfen, ob die für den pseudoelastischen Anteil gemessene Inelastizität echt ist, wurde eine Messung am System $\mathrm{Na}(3 \mathrm{~S})-\mathrm{SF}_{6}$ und eine Messung am System Na-Xe unter so weit wie möglich identischen Bedingungen vorgenommen (Abb. 5.19). Exakt gleich lassen sich die kinematischen Bedingungen natürlich schon aufgrund der unterschiedlichen Masse von $\mathrm{Xe}$ und $\mathrm{SF}_{6}$ nicht wählen; dennoch liegt die Abweichung des vorhergesagten NewtonKreises innerhalb der Fehlergrenzen der Flugzeitmessung. Die Na-Xe-Messung zeigt im wesentlichen, analog zu Abschnitt 4.3.3, daß Apparatur und Auswertung in der Lage sind, die Geschwindigkeitsverteilung elastischer Streuung korrekt wiederzugeben. Abb. 5.20 zeigt den in innere Energie übertragenen Anteil an der Stoßenergie in Abhängigkeit vom Schwerpunktswinkel. Für größere als die aufgetragenen Winkel ist der pseudoelastische Anteil nahezu unmeßbar klein. Die Inelastizität setzt bei ca. $40^{\circ}$ ein und steigt dann etwa linear mit dem Winkel an. Der maximale Energieübertrag beträgt immerhin ca. $60 \%$ der verfügbaren Energie.

Das Auflösungsvermögen des Experiments reicht nicht aus, um einzelne Vibrations/Rotationsniveaus des $\mathrm{SF}_{6}$ zu identifizieren. Daher können auf experimentellem Wege keine weitergehenden Aussagen darüber gewonnen werden, wie sich die Anregung des $\mathrm{SF}_{6}$ in Rotations- und Vibrationsanregung aufteilt und welche Niveaus bevölkert werden.

Zum Vergleich soll eine Messung am System $\mathrm{SF}_{6}$-Ar [22] herangezogen werden, die bei vergleichbaren Stoßenergieen $(0.73 \mathrm{eV}$ und $1.05 \mathrm{eV})$ durchgeführt wurde. Dort wird, unabhängig von der Stoßenergie, ein Maximum des Energieübertrags bei $\Theta_{C M}=140^{\circ}$ gefunden. Daß das Maximum nicht bei völlig zentralen Stößen stattfindet, wird damit erklärt, daß bei leicht peripheren Stößen sehr wirksame Rotationsanregung stattfindet, während zentrale Stöße ausschließlich zu Vibrationsanregung führen. Aus theoretischen Überlegungen wird gefolgert, daß die übertragene Energie sich je zur Hälfte auf Vibrations- und Rotationsanregung aufteilt. Hierin liegt sicherlich auch die Erklärung, warum für $\mathrm{Na}^{-\mathrm{SF}_{6}}$ überhaupt inelastische Streuung mit relativ großen Energieüberträgen beobachtet werden kann und die entsprechenden Stöße nicht zur Reaktion führen.

Vergleicht man den Energieübertrag zwischen den Systemen, so fällt auf, daß die Inelastizität für $\mathrm{Na}_{-} \mathrm{SF}_{6}$ erst bei größeren Winkeln einsetzt. Die Abhängigkeit von $\Theta_{C M}$ ist für beide Systeme bis zum Maximum näherungsweise linear. Die in [22] beobachteten maxi- 
malen Energieüberträge sind ca. $35 \%$ für $0.73 \mathrm{eV}$ Stoßenergie und ca. $45 \%$ für $1.05 \mathrm{eV}$ Stoßenergie. Geht man von einem weiteren Anstieg mit der Stoßenergie aus, befindet sich der hier gemessene Wert von 60 \% in Übereinstimmung mit den zitierten Ergebnissen.

\section{Vergleich mit statistischen Energieverteilungen}

Zur Klassifikation der Geschwindigkeitsverteilungen der gestreuten Produkte soll die von Levine eingeführte Methode der a priori-Verteilungen [48, 81] herangezogen werden. Die $a$ priori-Verteilung ist diejenige Energieverteilung, die sich einstellt, wenn die zur Verfügung stehende Energie sich gleichmäßig auf die erreichbaren Vibrations-, Rotations- und Translationszustände verteilt. Die a priori-Verteilung kann in der RRHO-(rigid rotor harmonic oszillator)-Näherung berechnet werden [56], ohne alle involvierten Niveaus zu kennen. Es ergibt sich

$$
P_{0}\left(E_{T R}^{\prime}\right)=\sqrt{E_{T R}^{\prime}}\left(E_{G E S}-E_{T R}^{\prime}\right)^{N}
$$

wobei $E_{G E S}$ die insgesamt im Stoß zur Verfügung stehende Energie, also Stoßenergie und innere Energie der Stoßpartner, $E_{T R}^{\prime}$ die gesamte Translationsenergie nach dem Stoß (im Schwerpunktsystem) und $\mathrm{N}$ die Anzahl der beteiligten Freiheitsgrade ist. $N$ ergibt sich aus der Zahl der Freiheitsgrade des Systems. In der RRHO-Näherung ergibt sich je Molekül

$$
N=f_{V}+\frac{f_{R}}{2}-1,
$$

wobei $f_{V}$ die Zahl der Vibrationsfreiheitsgrade, also der Normalschwingungen, und $f_{R}$ die Zahl der Rotationsfreiheitsgrade ist [56]. Das Maximum und die Halbwertsbreite für die so berechneten a priori-Verteilungen ist nach entsprechender Transformation in den Geschwindigkeitsraum in die Newton-Diagramme für reaktive Streuung eingetragen (Abbildungen 5.9, 5.11, 5.15 und 5.17). Der Abstand der Geschwindigkeitsverteilung der Produkte soll hier qualitativ als ein Maß für den Grad der Gleichverteilung der Energie während der Reaktion aufgefaßt werden.

$\mathrm{Zu}$ beobachten ist, daß die Produkte für die Streuung im Grundzustand im mittleren Winkelbereich eine etwa der a priori-Verteilung entsprechende $(0.6 \mathrm{eV})$ oder darunterliegende $(2 \mathrm{eV})$ Geschwindigkeit aufweisen; in den Bereichen der Vorwärts- und Rückwärtsstreuung sind die Produkte deutlich schneller. Verglichen mit den Winkelverteilungen, die konstante Beiträge im mittleren Winkelbereich und mehr oder weniger stark ausgeprägte Maxima für Vorwärts- und Rückwärtsstreuung aufweisen, ergibt sich die Vermutung, daß im Bereich mittlerer Winkel Zerfallsprodukte eines Stoßkomplexes zu beobachten sind, während abstreifende Stöße (Vorwärtsstreuung) und zentrale Stöße (Rückwärtsstreuung) zu direkteren Reaktionen führen.

Für den Fall elektronischer Anregung werden für beide Stoßenergieen Geschwindigkeiten beobachtet, die deutlich höher liegen als statistisch vorhergesagt. Im Gegensatz zum Grundzustand ist die Geschwindigkeitsverteilung weniger winkelabhängig; die Winkelabhängigkeit wird als nicht signifikant angesehen. Eine Koinzidenz zwischen dem Verhalten der Geschwindigkeitsverteilung und der Winkelverteilung wie im Grundzustand wird nicht beobachtet. 
Insgesamt folgt keine eindeutige Entscheidung über den Reaktionstyp des Systems aus den angeführten Beobachtungen. Vielmehr liegt die Vermutung nahe, daß einige Stöße zur Komplexbildung führen, andere jedoch zur direkten Reaktion; möglicherweise ist dies abhängig von der individuellen Konfiguration im Stoß.

\section{Qualitativer Vergleich mit Potentialrechnungen}

Für Na-SF 6 wurden für den Grundzustand und den ersten elektronisch angeregten Zustand des Gesamtsystems von B. Heumann [14] mit quantenchemischen Methoden Potentialflächen berechnet (Abb. 5.21 und 5.22). Die Details der Rechnung sind in [14] dargestellt. Es zeigt sich, daß die Potentialfläche für den angeregten Zustand im Hinblick auf die Reaktion streng repulsiv ist. Eine Reaktion ist tatsächlich nur infolge eines nichtadiabatischen Überganges möglich. Der wesentliche Faktor für das Auftreten einer Reaktion mit Na(3P) ist also die Übergangswahrscheinlichkeit zwischen den Potentialflächen. Ist der Übergang vollzogen, steht dem System zusätzlich zur Stoßenergie noch die Energiedifferenz zwischen den beiden Flächen zur Verfügung. Diese beträgt für den minimalen Abstand $1 \mathrm{eV}$. Das System zeigt qualitativ auch das entsprechende Verhalten : Im Falle der Streuung an $\mathrm{Na}(3 \mathrm{P})$ ist deutlich mehr kinetische Energie nach dem Stoß vorhanden als im Grundzustand. Allerdings sind die realen Verhältnisse durch die Übertragung eines erheblichen Energieanteils in die Vibrationsfreiheitsgrade des $\mathrm{SF}_{5}$ geprägt. Dieses Verhalten kann die Potentialfläche durch die starre Modellierung des $\mathrm{SF}_{5}$ nicht wiedergeben. Für niedrige Stoßenergien spielt die Reaktionsbarriere der Grundzustandsfläche eine Rolle. Durch die zusätzliche Energie kann diese für $\mathrm{Na}(3 \mathrm{P})$ leichter überwunden werden. Dementsprechend sieht man für die niedrigere Stoßenergie eine deutliche Zunahme des Querschnittes, für die höhere hingegen nicht. 


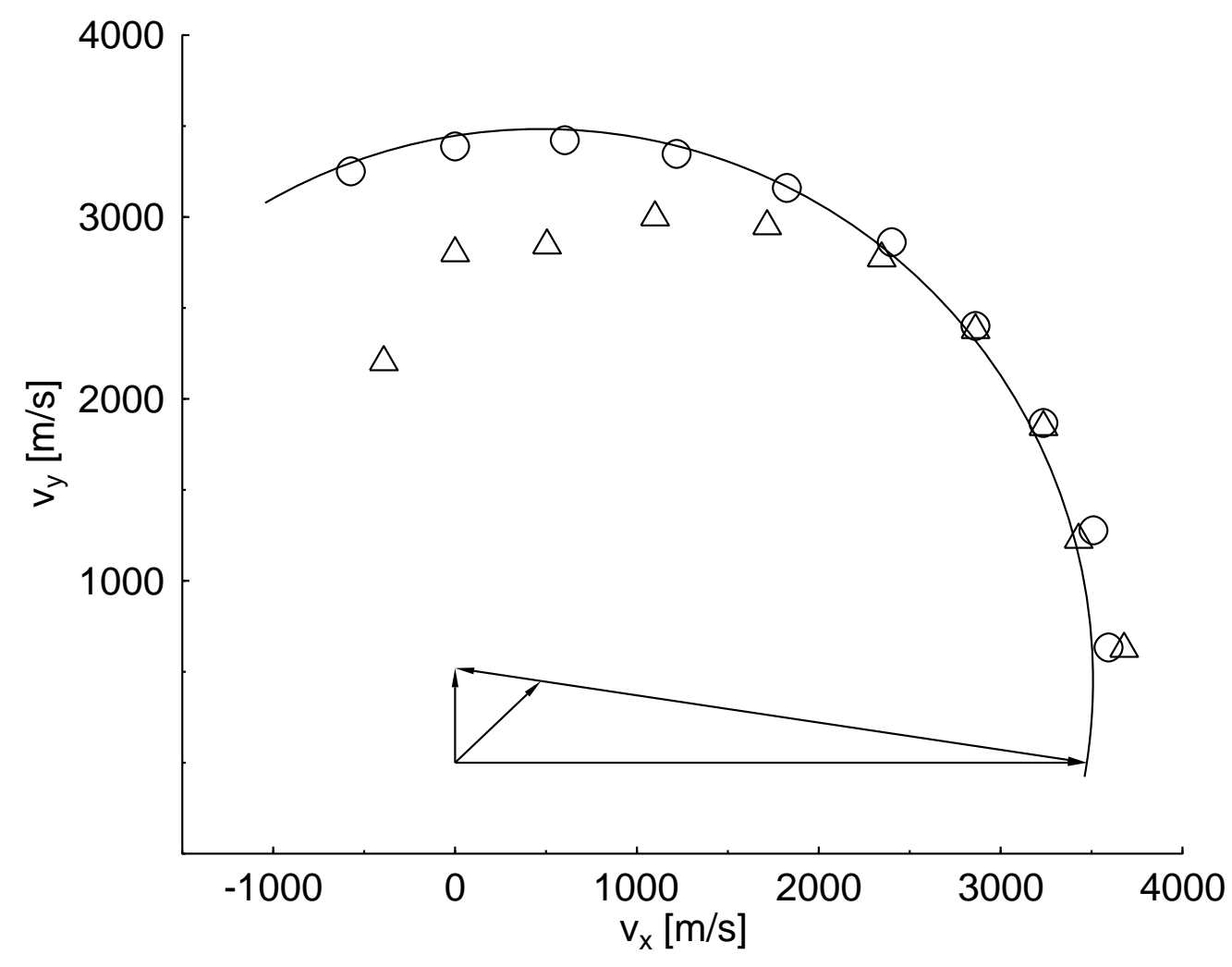

Abb. 5.19: Auftragung der elastischen bzw. inelastischen Streuung von $\mathrm{Na}$ an $\mathrm{SF}_{6}$ bei einer Stoßenergie von $1.3 \mathrm{eV}(\triangle)$. Zum Vergleich ist das Ergebnis einer Messung an $\mathrm{Na}-\mathrm{Xe}(\mathrm{o})$ aufgetragen, die unter möglichst vergleichbaren Bedingungen durchgeführt wurde. Der durchgezogene Kreis deutet die korrekte Geschwindigkeit für $\mathrm{Na}_{-} \mathrm{SF}_{6}$ an. 


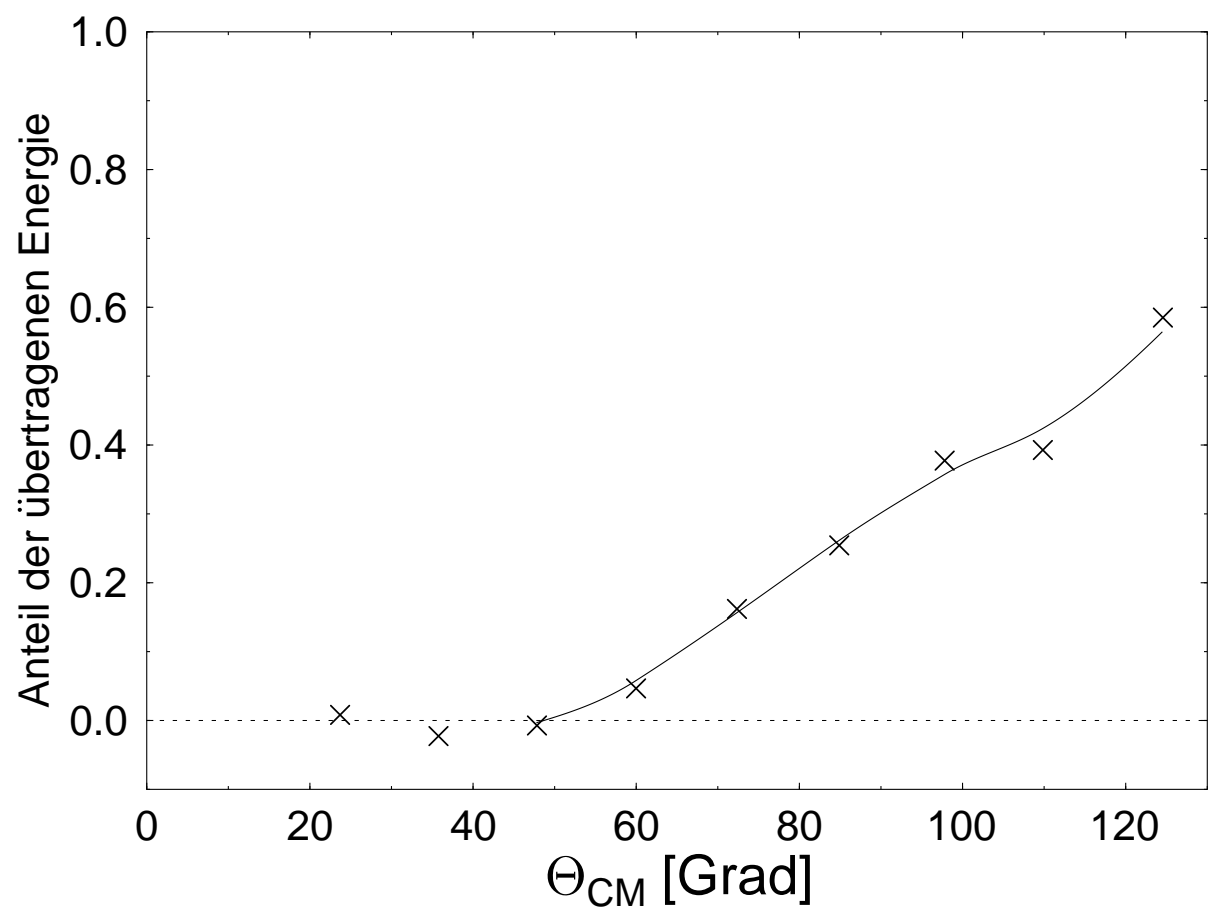

Abb. 5.20: Anteil der in innere Energie des $\mathrm{SF}_{6}$ transferierten Stoßenergie.

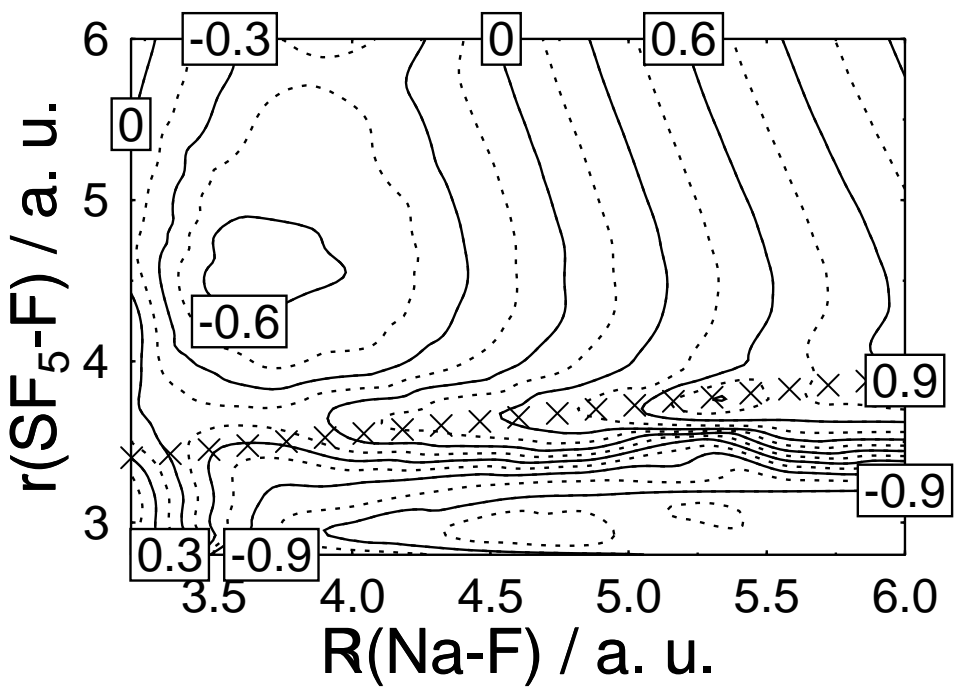

Abb. 5.21: Potentialfläche für den Grundzustand. 


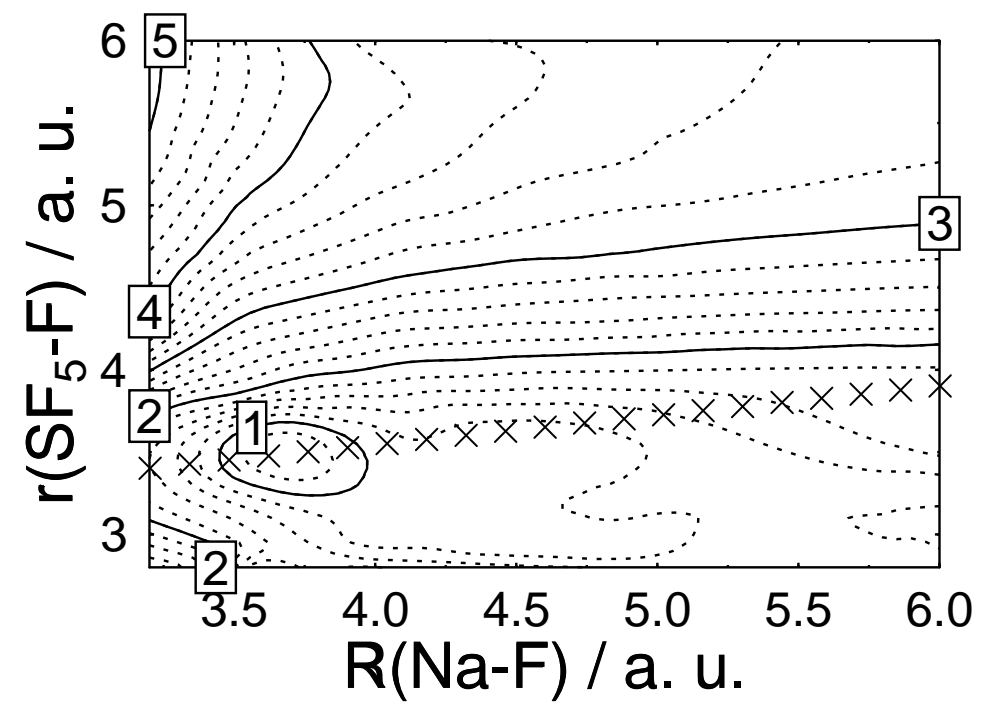

Abb. 5.22: Potentialfläche für den ersten angeregten Zustand (Angabe des Potentials in eV). In der Rechnung mit der CASSCF-Methode wurden ausschließlich die dargestellten Koordina-

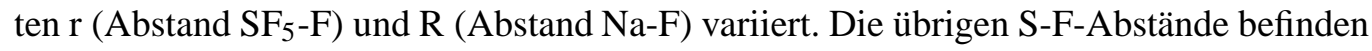
sich für $\mathrm{SF}_{6}$ im Gleichgewicht. Für die Flächen wurde eine Analyse der Ladungsdichte in der Umgebung der einzelnen Atome durchgeführt (Mulliken charge analysis). Danach läßt sich die Grundzustandsfläche in einen neutralen (Bereich unterhalb der gekreuzten Linie) und einen ionischen Bereich aufteilen (bezüglich des Natriums und des korrespondierenden Fluoratoms). Wird diese Grenzlinie überschritten, findet im Grundzustand Reaktion statt (harpooning model). Die angeregte Fläche enthält keinen ionischen Anteil. Für eine Reaktion ist ein Übergang in den Grundzustand notwendig. Eine genauere Beschreibung der technischen und interpretativen Details findet sich in [14]. 


\section{Kapitel 6}

\section{Streuung von $\mathrm{SiF}_{4}$ an $\mathrm{Na}(3 \mathrm{~S})$ und $\mathrm{Na}(3 \mathrm{P})$}

\section{1 Überblick}

\section{Motivation}

Um dem reaktiven System $\mathrm{Na}(3 \mathrm{~S}), \mathrm{Na}(3 \mathrm{P})$ - $\mathrm{SF}_{6}$ ein nichtreaktives mit ansonsten ähnlichen Eigenschaften gegenüberzustellen, wurden Messungen der Streuung von $\mathrm{SiF}_{4}$ an $\mathrm{Na}(3 \mathrm{~S})$ und $\mathrm{Na}(3 \mathrm{P})$ durchgeführt. $\mathrm{SiF}_{4}$ ist mit seiner Tetraederform ebenfalls hochsymmetrisch, weist allerdings keine ganz so hohe Symmetrie wie $\mathrm{SF}_{6}$ auf.

Wie bei $\mathrm{SF}_{6}$ ist eine Vibrationsanregung von $\mathrm{SiF}_{4}$ mit dem vorhandenen $\mathrm{CO}_{2}$-Laser [62] möglich (entsprechend den in [55] dargestellten Experimenten an $\mathrm{Na}-\mathrm{SF}_{6}^{\dagger}$ ). Für die 9P40Linie des $\mathrm{CO}_{2}$-Lasers existiert eine Koinzidenz mit der $v_{3}$-Mode des $\mathrm{SiF}_{4}\left(1029 \mathrm{~cm}^{-1}\right)$. Diese Anregung konnte auch experimentell durchgeführt werden; es wurde ausschließlich auf der 9P40-Linie ein Anregungssignal mit dem Bolometer (s. Abschnitt 3.5 gemessen, dessen Intensität im Vergleich mit Messungen an $\mathrm{SF}_{6}$ eine Anregung mit einem Photon pro Molekül ergibt. Dieser Anregungsprozess wurde für die hier dargestellten Experimente nicht ausgenutzt. Es ist fraglich, ob die erreichbare Effizienz Streumessungen mit $\mathrm{SiF}_{4}^{\dagger}$ erlaubt.

Nebenbei sei bemerkt, daß Natrium und $\mathrm{SiF}_{4}$ unter „,Reagenzglasbedingungen“ durchaus miteinander reagieren. Großtechnisch wird die Reaktion

$$
4 \mathrm{Na}+\mathrm{SiF}_{4} \rightarrow 4 \mathrm{NaF}+\mathrm{Si}
$$

für die Produktion reinen Siliziums zur Verwendung in Solarzellen eingesetzt [69].

\section{Mögliche Ausgangskanäle}

Für Stöße von Na im elektronischen Grundzustand ergibt sich für die - analog dem System $\mathrm{Na}-\mathrm{SF}_{6}$ - mögliche Reaktion

$$
\mathrm{Na}+\mathrm{SiF}_{4} \rightarrow \mathrm{NaF}+\mathrm{SiF}_{3}
$$




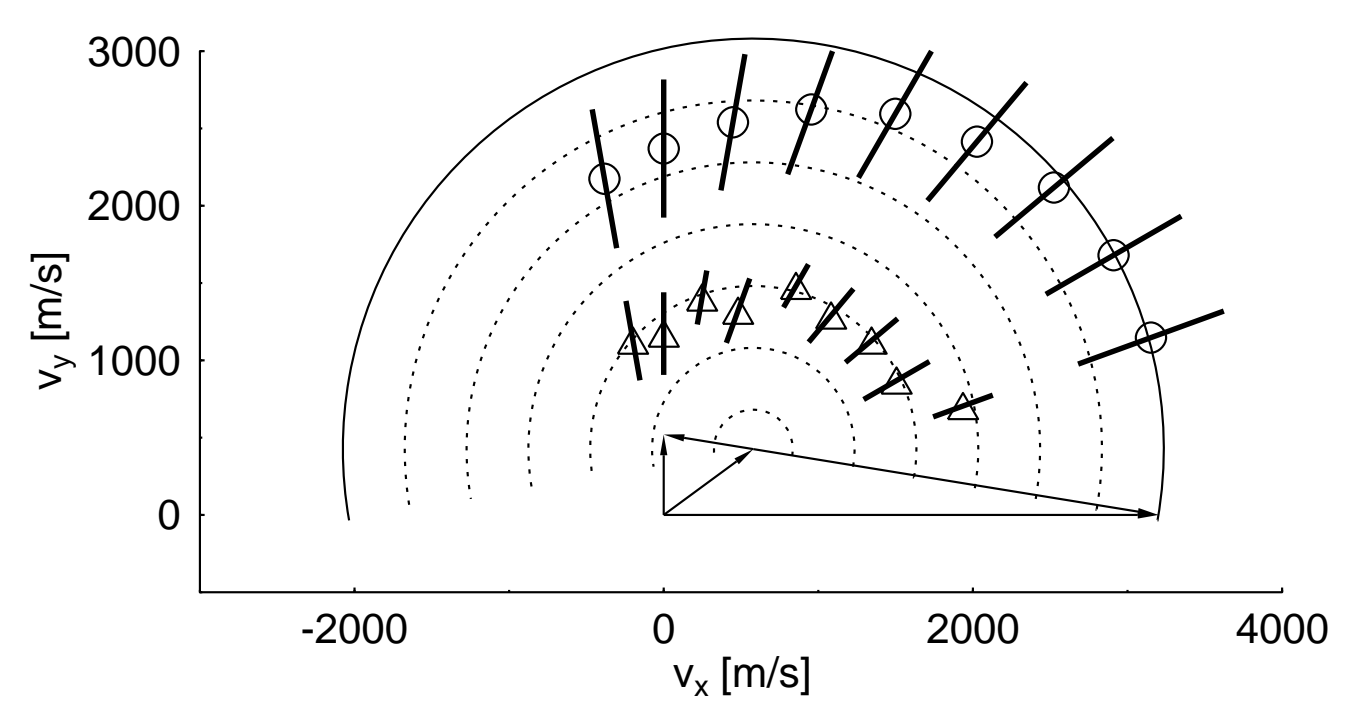

Abb. 6.1: Newton-Diagramm für die Streuung im Grund- und angeregten Zustand. Die Symbole $(\bigcirc$ : $\mathrm{Na}(3 \mathrm{~S})-\mathrm{SiF}_{4}, \triangle: \mathrm{Na}(3 \mathrm{P})-\mathrm{SiF}_{4}$, langsamer Anteil) geben die mittleren Geschwindigkeiten, die Balken die Halbwertsbreite der angepaßten Funktionen wieder.

aus thermochemischen Daten [10] eine Reaktionsenthalpie von $+239 \frac{\mathrm{kJ}}{\mathrm{mol}}$, entsprechend $2.48 \mathrm{eV}$. Dieser Wert kann mit den hier verwendeten Stoßenergien nicht erreicht werden. Damit bleiben nur elastische und inelastische Streuprozesse möglich. Für $\mathrm{Na}(3 \mathrm{P})$ mit einer zusätzlichen inneren Energie von $2.1 \mathrm{eV}$ ist der reaktive Kanal prinzipiell zugänglich. Es ergeben sich die folgenden Möglichkeiten :

$$
N a(3 P)+S_{F} F_{4}\left(v=v_{0}\right) \rightarrow \begin{cases}N a(3 P)+S_{F} F_{4}\left(v=v_{0}\right) & \text { elastisch (a) } \\ N a(3 P)+S i F_{4}\left(v=v^{\prime}\right) & \text { inelastisch (b) } \\ N a(3 S)+S i F_{4}\left(v=v^{\prime \prime}\right) & \text { E-VRT-Übergang, ,Löschung“ (c) } \\ N a F+S i F_{3} & \text { reaktiv (d) }\end{cases}
$$

Wie im folgenden dargelegt wird, findet Prozeß (d) nicht statt. Gegenstand der Untersuchung wird im wesentlichen Kanal (c) sein.

\subsection{Experimentelle Ergebnisse}

\section{Bedingungen}

Die Geschwindigkeit des Natriumstrahls für die Messungen an $\mathrm{Na}(3 \mathrm{~S})-\mathrm{SiF}_{4}$ und $\mathrm{Na}(3 \mathrm{P})-$ $\mathrm{SiF}_{4}$ war $3200 \frac{\mathrm{m}}{\mathrm{s}}$ bei einer Halbwertsbreite von $400 \frac{\mathrm{m}}{\mathrm{s}}$. Für die berechnete Sekundärstrahlge- 
schwindigkeit ergibt sich $657 \frac{\mathrm{m}}{\mathrm{s}}$ (s. Abschnitt 2.5); es wurde eine molare Wärmekapazität von

$c_{p}=73.6 \frac{\mathrm{J}}{\mathrm{mol} \cdot \mathrm{K}}[10]$ verwendet. Damit ergibt sich die Stoßenergie im Schwerpunktsystem zu $1.0 \mathrm{eV}$. Der Abstand zwischen der Sekundärdüse und dem Primärstrahl betrug $20 \mathrm{~mm}$, die Temperatur der Sekundärdüse $200{ }^{\circ} \mathrm{C}$.

Die Präparation des $\mathrm{Na}(3 \mathrm{P})$ wurde unter Verwendung der Zwei-Linien-Anregung, jedoch im Vergleich zu den Experimenten mit $\mathrm{SF}_{6}$ mit geringerer Leistung durchgeführt, da zur pseudostatistischen Modulation die Pockelszelle verwendet wurde. Die experimentelle Anregungseffizienz $\eta$ wurde aus reaktiven Streumessungen an $\mathrm{Na}(3 \mathrm{P})-\mathrm{SF}_{6}$ entsprechend Abschnitt $4.1 \mathrm{zu} 15 \%$ bestimmt. Eine Überprüfung von $\eta$ aus den Messungen an $\mathrm{Na}(3 \mathrm{P})$ $\mathrm{SiF}_{4}$ ist nicht möglich.

Die Flugzeitspektren für den Grundzustand wurden mit der maximal möglichen Auflösung des TOF-Systems (Kanalbreite $4 \mu s$ ) gemessen. Für die Differenzspektren wurde zugunsten des Signal-Rausch-Verhältnisses eine Kanalbreite von 10 $\mu$ s verwendet. Die Rechtfertigung des Verfahrens ist in Abschnitt 4.3 gegeben.

Die Messungen erstrecken sich über einen Winkelbereich von $20^{\circ}$ bis $100^{\circ}$ im Laborsystem. Unterhalb von $20^{\circ}$ ist die Zählrate der Streuung im Grundzustand so groß, daß Maßnahmen zur Verringerung der Empfindlichkeit des Detektorsystems ergriffen werden müssen. Das daran gemessen kleine Differenzsignal ist dann nicht mehr nachweisbar. Die obere Grenze von $100^{\circ}$ liegt in Bauteilen der Vakuumapparatur begründet. Die sich daraus ergebende Verteilung der Flugzeitspektren im Newtondiagramm ist in Abb. $6.1 \mathrm{zu}$ sehen.

\section{Ergebnisse - Grundzustand}

Für kleine Winkel wird nur elastische Streuung beobachtet. Die Breite der Verteilung im Flugzeitspektrum (Abb. 6.2) ist ausschließlich in apparativen Effekten (Geschwindigkeitsverteilung der Strahlen etc. entsprechend Abschnitt 4.3.2) begründet. Für größere Winkel ergibt sich eine Tendenz zu inelastischer Streuung. Der Übergang von elastischer Streuung für kleine Winkel zu inelastischer Streuung bei großen Winkeln erfolgt kontinuierlich. Einzelne Vibrationsniveaus können nicht aufgelöst werden. Der Vergleich mit Messungen definitiv elastischer Streuung (Abschnitt 4.3.3) zeigt, daß die Abweichung zu elastischer Streuung signifikant ist. Die Abweichung läßt sich auch nicht durch Annahme einer geringeren Sekundärstrahlgeschwindigkeit erklären, da sich das Newtondiagramm für den Nachweis des Natriums nur wenig mit der Sekundärstrahlgeschwindigkeit ändert. Damit zeigt sich dasselbe Verhalten, wie es auch für $\mathrm{Na}_{-} \mathrm{SF}_{6}$ (voriges Kapitel) oder $\mathrm{Ar}-\mathrm{SF}_{6}$ [22] bei einer vergleichbaren Stoßenergie beobachtet wird.

Der maximale gemessene Energieübertrag (bei $\theta_{L a b}=100^{\circ}$ ) ist $0.42 \mathrm{eV}$. Abb. 6.4, oben, zeigt die Winkelverteilung. Angesichts der relativ hohen Stoßenergie liegt der beobachtete Bereich jenseits etwaiger Regenbogenoszillationen und zeigt dementsprechend keine Struktur.

\section{Ergebnisse - Na(3P)}

Die für die Streuung mit angeregtem Natrium gemessenen Differenz-Flugzeitspektren (Abb. 6.3) setzen sich aus zwei Anteilen zusammen: 


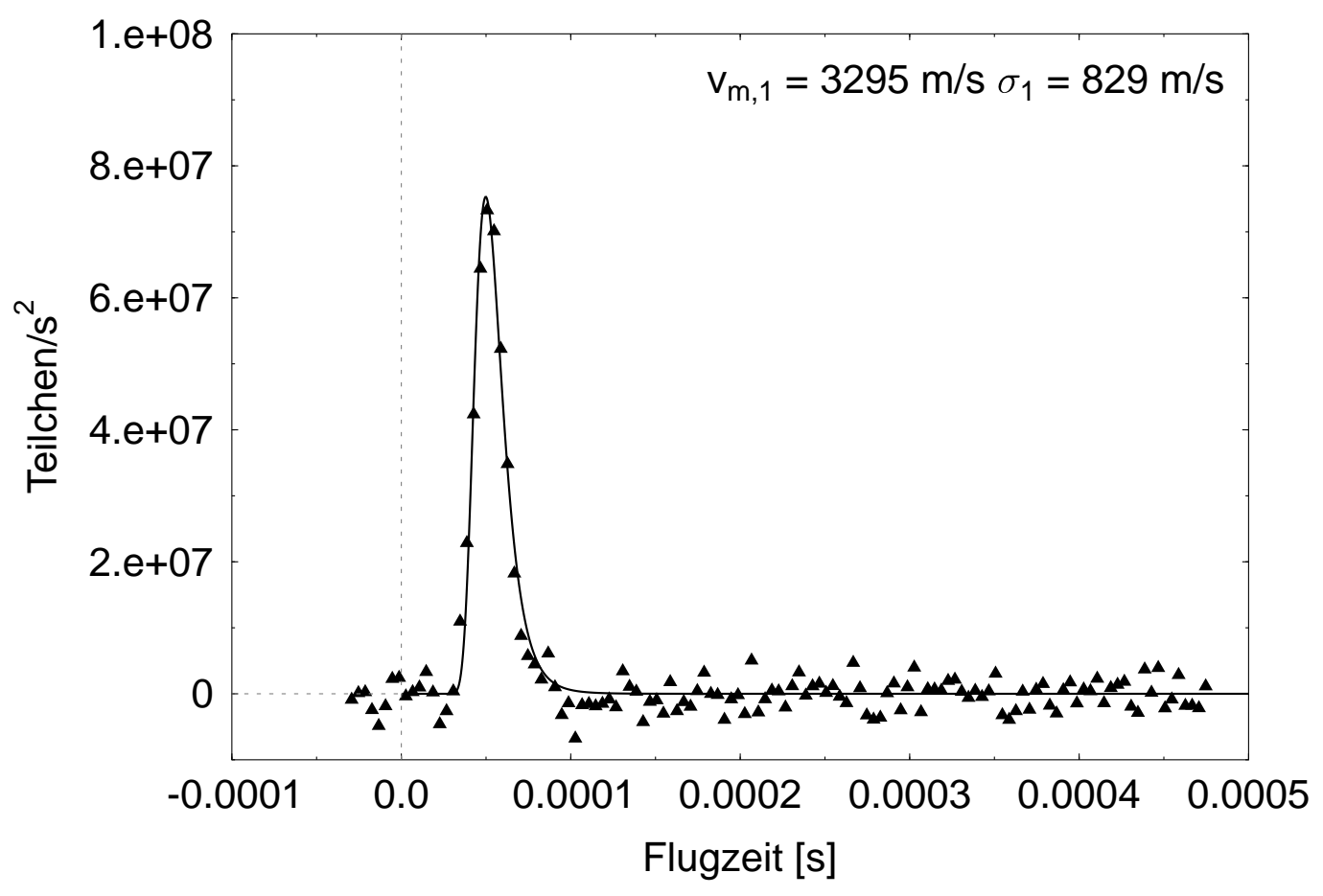

Abb. 6.2: Flugzeitspektrum der Streuung im Grundzustand auf dem Laborwinkel $\theta_{L a b}=40^{\circ}$. 


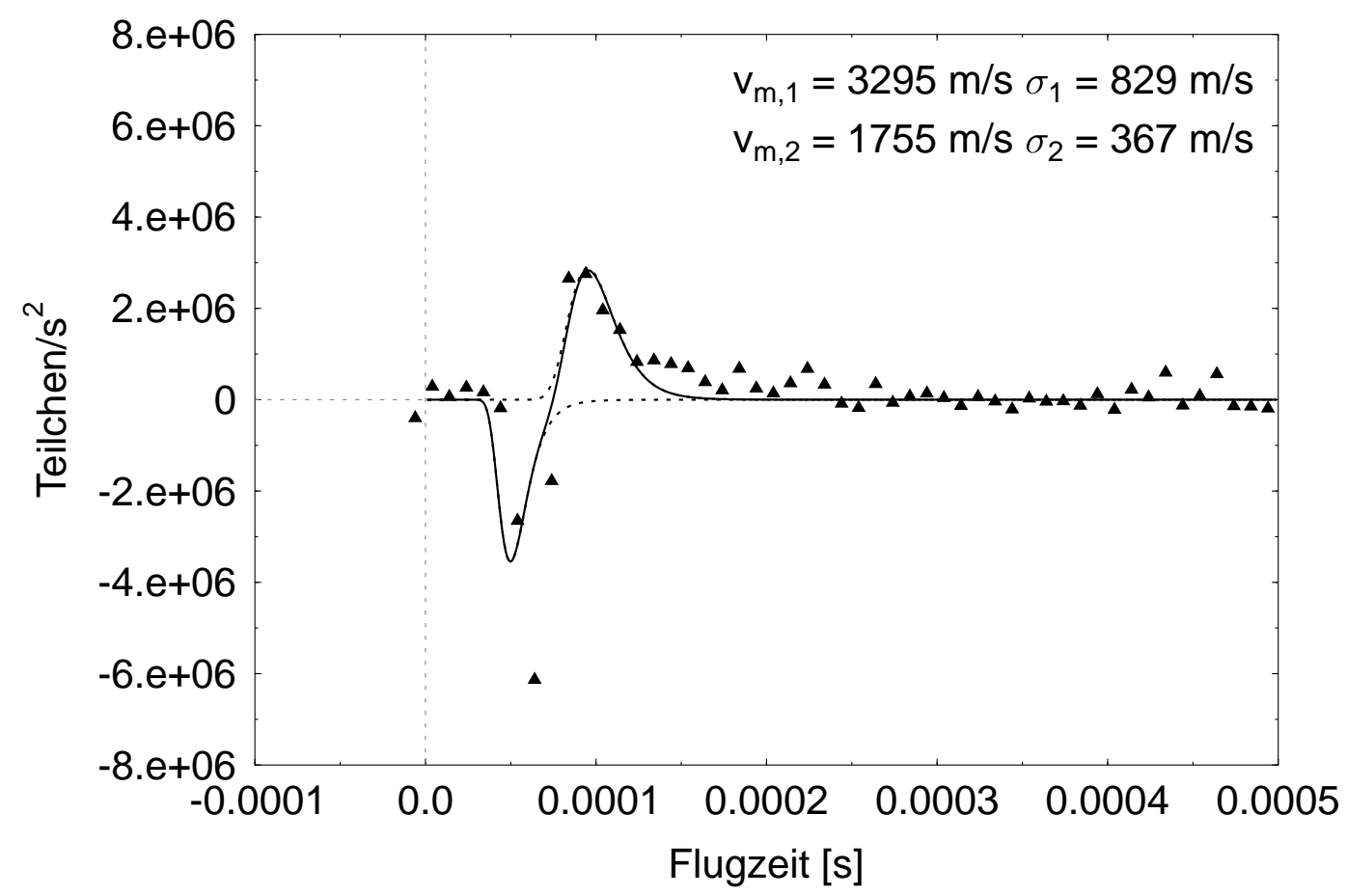

Abb. 6.3: Flugzeitspektrum für die Streuung im angeregten Zustand für $\theta_{L a b}=40^{\circ}$. Bei der Anpassung der Funktionen wurde davon ausgegangen, daß sich das Spektrum aus einem (negativen) Anteil, der dem Grundzustandsspektrum entspricht, sowie einer weiteren transformierten Gauß-Funktion besteht. 


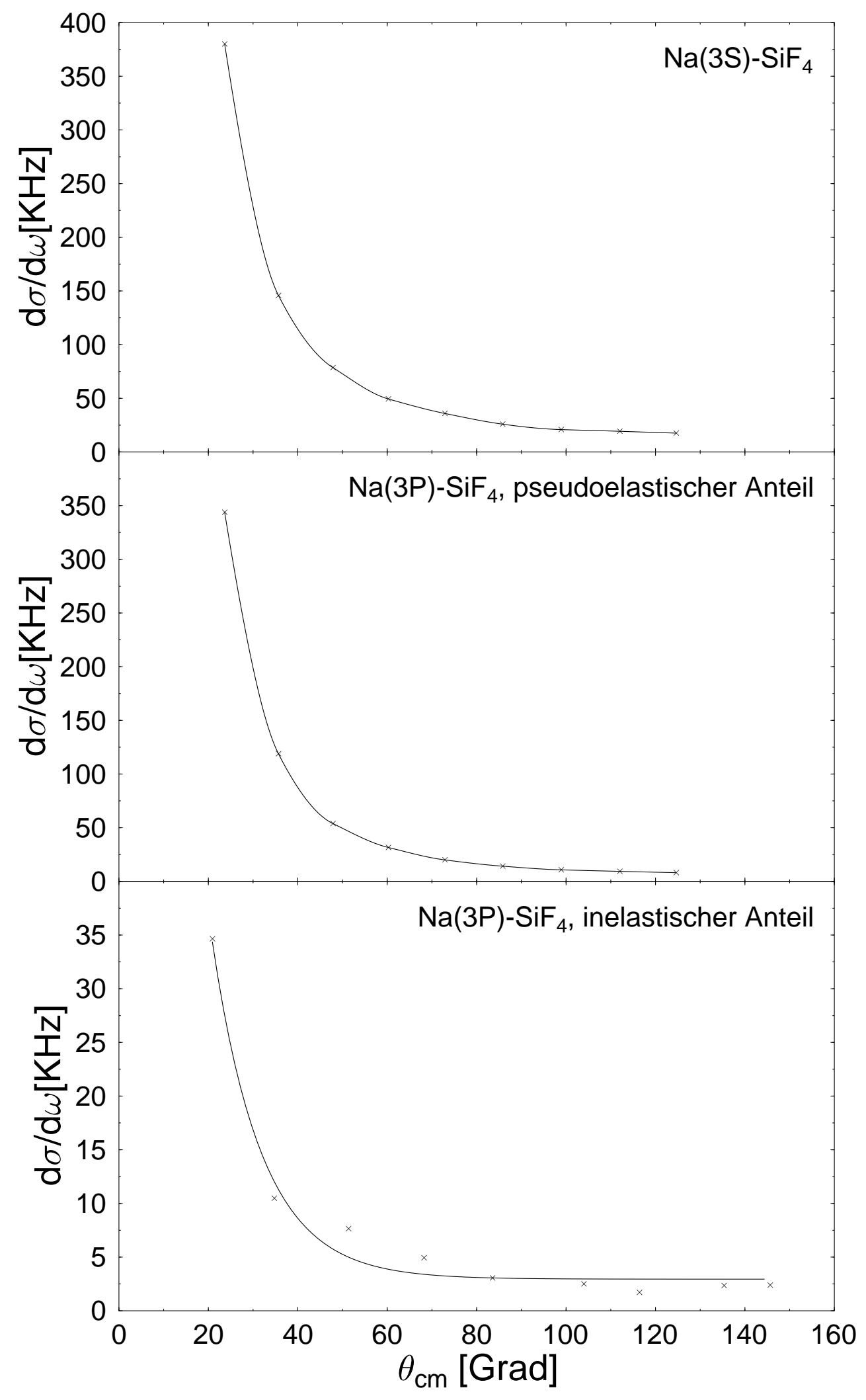

Abb. 6.4: Winkelverteilungen für die verschiedenen Anteile, bestimmt durch Integration der Flugzeitspektren. Die Transformation ins Schwerpunktsystem wurde mit den Mittelwerten der Geschwindigkeit durchgeführt. 
- Der vordere, negative Anteil gehört zur auch im Grundzustand beobachteten, elastischen bzw. inelastischen Streuung. Die Spektren zeigen eine Reduktion des Querschnitts für diesen Kanal im Vergleich zum Grundzustand. Für diesen Anteil kann im Experiment nicht entschieden werden, ob elastische Streuung oder resonanter Energietransfer, bei dem exakt die Anregungsenergie des Natriums übertragen wird, stattfindet.

- Zu größeren Flugzeiten hin wird ein positiver Anteil beobachtet; für die entsprechenden Zeiten ergibt die Streuung im Grundzustand kein Signal. Dieser Anteil wird durch Verwendung des LIF-Detektors ebenfalls als nichtreaktiv gestreutes $\mathrm{Na}$ identifiziert. Als Erklärung kommt also reaktive Streuung nicht in Betracht. Damit liegt eine Deutung durch einen Löschprozess (s. o.) nahe.

Diese Struktur wird für alle gemessenen Flugzeitspektren beobachtet. Die Intensitätsverhältnisse ändern sich zu großen Winkeln hin zugunsten des langsamen Anteils. Für die Auswertung wurden zunächst an die Spektren für den Grundzustand transformierte Gaußverteilungen angepaßt. Für die Messungen im angeregten Zustand wurde davon ausgegangen, daß diese sich aus der in der Amplitude skalierten Grundzustandsverteilung und einer weiteren transformierten Gaußfunktion zusammensetzen. Damit sind für den Grundzustand drei Parameter (eine Gaußfunktion) und für den angeregten Zustand vier Parameter (eine Gaußfunktion und eine Amplitude) anzupassen. Die angepaßte Kurve gibt die Flugzeitspektren in allen Fällen gut wieder. Die erhaltenen mittleren Geschwindigkeiten und Breiten sind graphisch in Abb. 6.1 eingetragen. Man erkennt, daß der zweite Anteil für alle Winkel - mit einer leichten Abweichung für $\theta_{L a b}=20^{\circ}$ - die gleiche mittlere Geschwindigkeit aufweist.

Unter Berücksichtigung der Anregungseffizienz von $15 \%$ lassen sich für die beiden Anteile relativ zum Grundzustand differentielle Querschnitte berechnen, die in Abb. 6.4 dargestellt sind. Die Winkelverteilung für den elastischen/inelastischen Anteil weicht nur wenig von der für den Grundzustand ermittelten ab. Für den langsamen Anteil zeigt sich ein konstanter Querschnitt für mittlere bis große Winkel, zu kleinen Winkeln hin steigt der Querschnitt an.

\subsection{Statistische Interpretation der Meßdaten}

Wie im vorigen Abschnitt gesagt, wird der langsame Anteil der Flugzeitspektren, der bei Streuung an $\mathrm{Na}(3 \mathrm{P})$ beobachtet wird, durch einen Löschprozess

$$
N a(3 P)+\operatorname{SiF}_{4}\left(v=v_{0}\right) \rightarrow N a(3 S)+S i F_{4}\left(v=v^{\prime}\right)
$$

gedeutet. Bei einem solchen Prozeß findet im Stoß eine Abregung von $\mathrm{Na}(3 \mathrm{P})$ nach $\mathrm{Na}(3 \mathrm{~S})$ statt. Die dadurch verfügbare elektronische Anregungsenergie wird auf die Vibrations- und Rotationsfreiheitsgrade des Moleküls sowie die Translationsenergie beider Stoßpartner übertragen; daher spricht man von einem E $\rightarrow$ VRT-Übergang [48]. Die theoretisch optimale Behandlung eines solchen nicht-adiabatischen Überganges wäre die Ermittlung der entsprechenden Potentialflächen und die Untersuchung des Überganges mit Hilfe derselben wie 


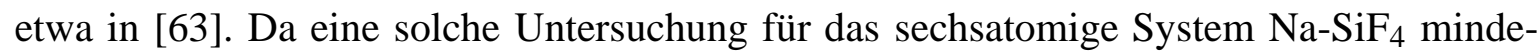
stens sehr aufwendig ist und auf jeden Fall die für diese Arbeit verfügbaren Möglichkeiten übersteigt, soll die Interpretation mit einem einfachen statistischen Modell erfolgen.

Analog zu [81, 34] wird dabei das von Bernstein und Levine [48] eingeführte Verfahren der Berechnung von a priori-Verteilungen verwendet. Es wird davon ausgegangen, daß alle energetisch erlaubten Zustände des Gesamtsystems - hier sind das Vibrations-, Rotationsund Translationszustände - dieselbe Besetzungswahrscheinlichkeit haben. Die Zustandsdichte für eine bestimmte Aufteilung der Energie des Systems gibt dann die Wahrscheinlichkeit $P_{0}$ dieser Energieaufteilung an.

Im Experiment kann nur die Aufteilung der Gesamtenergie des Systems in Translationsenergie und innere Energie des $\mathrm{SiF}_{4}$ bestimmt werden. Eine Auflösung der Vibrationsniveaus ist wegen des geringen Abstands der Niveaus bei $\mathrm{SiF}_{4}$ durch Flugzeitexperimente nicht möglich. Zur Interpretation der Messungen ist die entsprechende a priori-Verteilung zu berechnen. In der Näherung, daß das $\mathrm{SiF}_{4}$-Molekül als starrer Rotator angesehen wird und die Vibrationen durch gekoppelte harmonische Oszillatoren modelliert werden (RRHO-Näherung), ergibt sich für die a priori-Verteilung der Translationsenergie [48, 34]

$$
P_{0}\left(E_{t r, c m}^{\prime}\right) \sim \sqrt{E_{t r, c m}^{\prime}}\left(E_{t r, c m}+E_{e x}-E_{t r, c m}^{\prime}\right)^{N}
$$

dabei ist $E_{t r, c m}^{\prime}$ die gesamte Translationsenergie des Systems nach dem Stoß im Schwerpunktsystem, $E_{t r, c m}$ die gesamte Translationsenergie vor dem Stoß und $E_{e x}$ die Anregungsenergie des Natriums. $N$ ergibt sich entsprechend 5.5 aus der Zahl der Rotations- und Vibrationsfreiheitsgrade.

Abb. 6.5 zeigt einen Vergleich eines in das Schwerpunktsystem transformierten Flugzeitspektrums mit der aus 6.3 erhaltenen Verteilung. Der mittlere Energieübertrag (Position des Maximums) wird korrekt vorhergesagt. Um bessere Vergleichsmöglichkeiten zu schaffen, ist in Abb. 6.6 die aufgrund a priori-Verteilung berechnete Flugzeitverteilung an ein Flugzeitspektrum angepaßt dargestellt.

Voraussetzung für die Anwendung des dargestellten Modells ist, daß eine vollständige Gleichgewichtsverteilung über alle Freiheitsgrade des Systems erreicht wird. Umgekehrt kann also geschlossen werden, daß ein Stoßkomplex $\mathrm{NaSiF}_{4}$ gebildet werden muß, dessen Lebensdauer für die Einstellung des Gleichgewichts ausreicht. Die Annahme eines Stoßkomplexes ist auch mit dem für mittlere bis große Streuwinkel näherungsweise konstanten differentiellen Querschnitt konsistent. Für $\theta_{L a b}=20^{\circ}$ zeigt sich gleichzeitig mit einem Anstieg des Querschnitts eine größere kinetische Energie nach dem Stoß; dies würde bedeuten, daß der Prozeß zu kleineren Winkeln hin direkter abläuft.

\subsection{Vergleich mit anderen Experimenten}

Obwohl eine große Zahl an experimentellen Untersuchungen zum Energietransfer zwischen elektronischer und Rotations-/Vibrationsenergie in Molekularstrahlexperimenten vorliegt, gestaltet sich der Vergleich insofern als schwierig, als fast alle diese Untersuchungen als Stoßpartner des elektronisch angeregten Atoms ein zweiatomiges Molekül verwenden. In diesem Fall sind sowohl die experimentellen wie auch die interpretativen Möglichkeiten besser. Da nur ein Vibrationsfreiheitsgrad auftritt, kann aus der Translationsenergieverteilung 


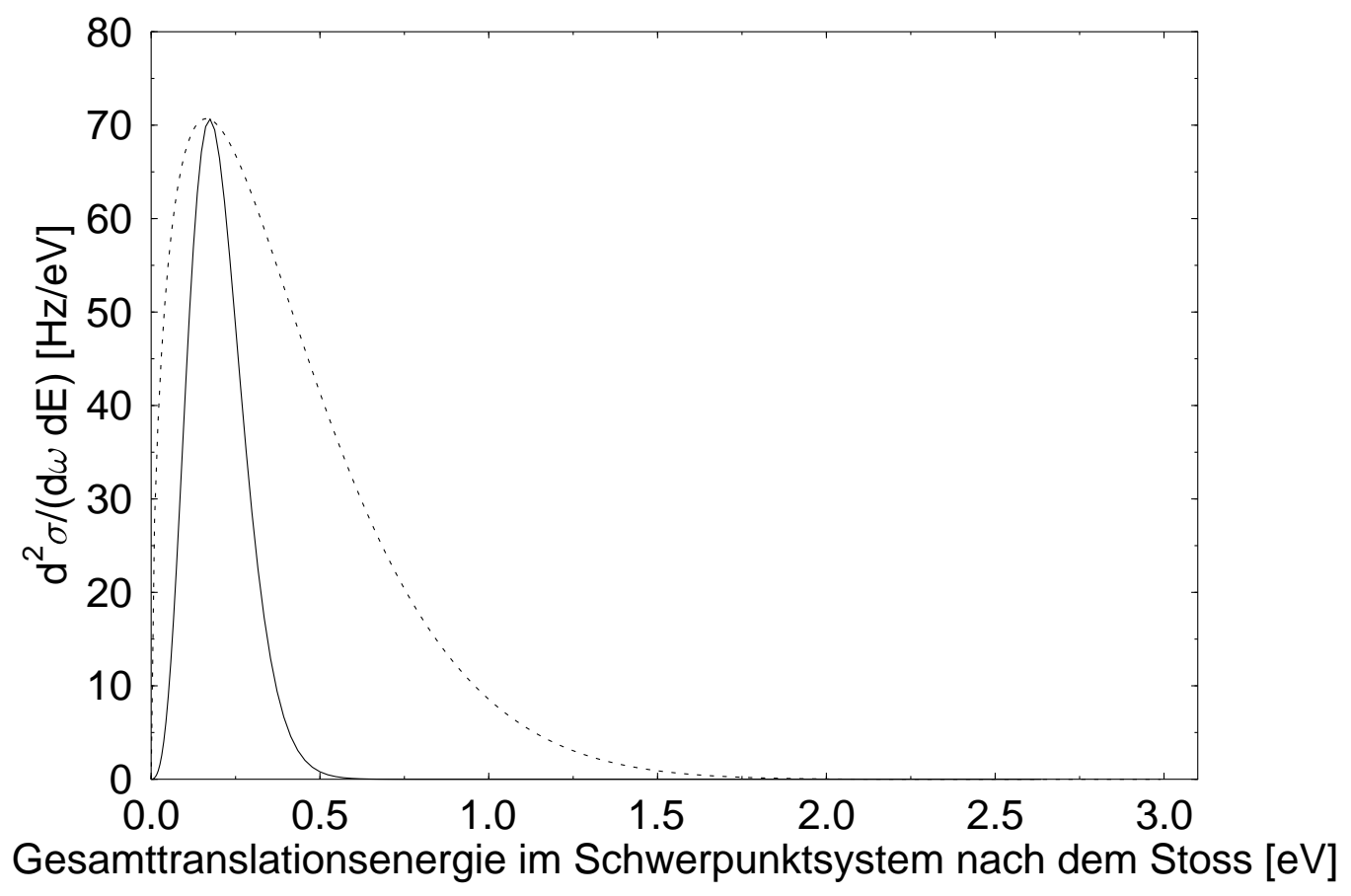

Abb. 6.5: Energieverteilung für das bei $\theta_{L a b}=40^{\circ}$ aufgenommene Flugzeitspektrum. Die durchgezogene Linie stellt das ins Schwerpunktsystem transformierte Flugzeitspektrum, repräsentiert durch die im „freien“ Fit erhaltenen Funktionen, dar; der zugehörige Schwerpunkswinkel variiert über einen Bereich von $40^{\circ}$ bis $50^{\circ}$. Die gestrichelte Linie gibt die Energieverteilung der a priori-Verteilung wieder.

unmittelbar auf die Anzahl übertragener Vibrationsquanten geschlossen werden; außerdem ist teilweise die Auflösung der Vibrationsniveaus in der Messung möglich. Weiterhin können zur Interpretation Potentialflächen berechnet werden.

Hier soll im wesentlichen auf die Fragen eingegangen werden, ob

- das hier vorgeschlagene Modell der Interaktion über einen zumindest eine Rotationsperiode überlebenden Stoßkomplex im Vergleich zu anderen Ergebnissen tragfähig ist,

- als wie erfolgreich sich der hier benutzte statistische Interpretationsansatz erweist und

- ob der hier nicht nachzuweisende resonante Energietransfer (s. Kap. 6.2) eine wesentliche Rolle spielt.

Alle im folgenden angegebenen Experimente laufen nach dem Schema

$$
\text { Atom }^{*}+\text { Molekül } \rightarrow \text { Atom }+ \text { Molekül }^{\dagger}
$$




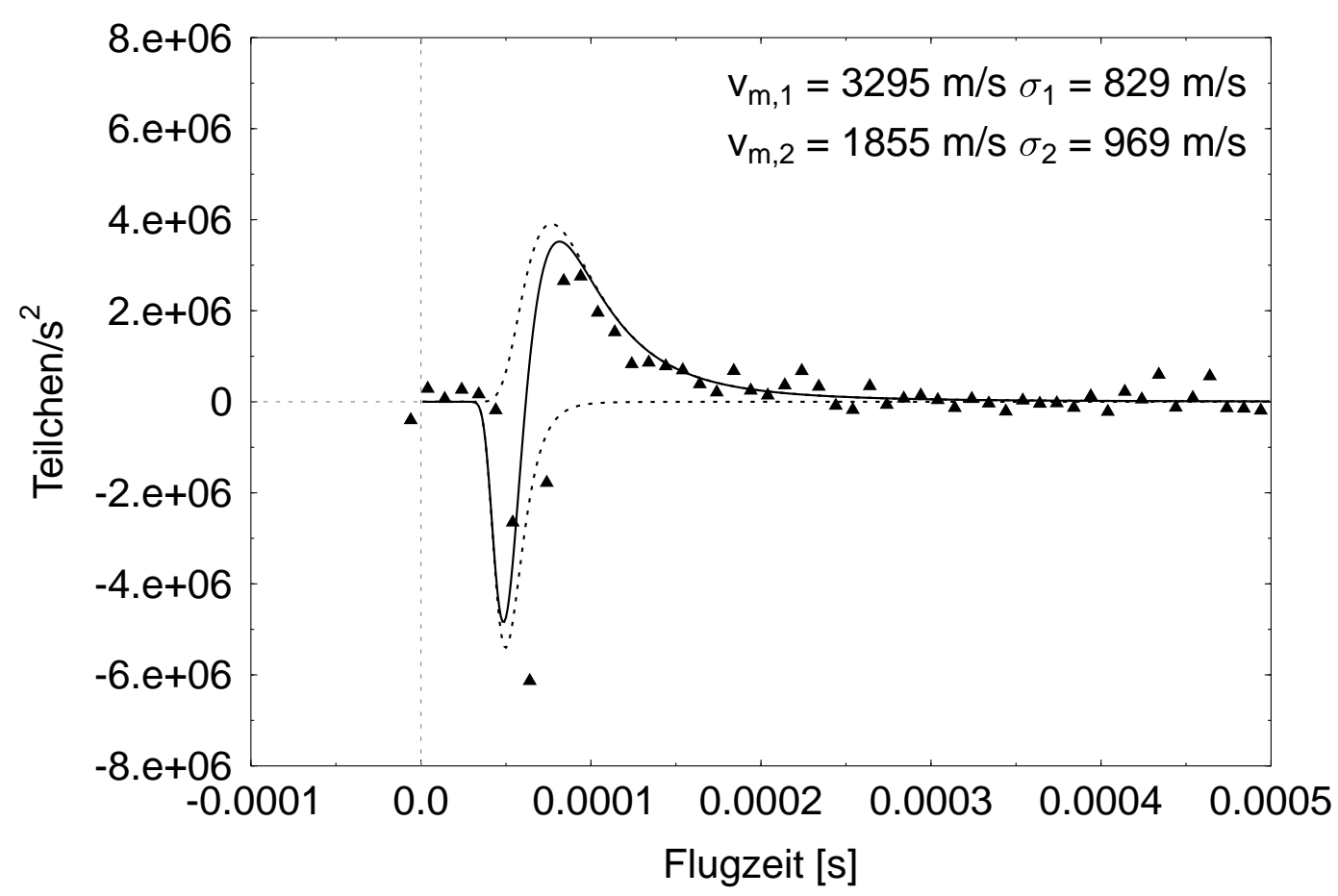

Abb. 6.6: Noch einmal das Flugzeitspektrum aus Abb. 6.3. Die angepaßte Funktion besteht aus der Grundzustandsverteilung und - für den inelastischen Anteil - der a priori-Verteilung. Es ergibt sich eine deutliche Verbreiterung des auf Löschung zurückzuführenden Anteils gegenüber Abb. 6.3 in die Flugzeitverteilung des pseudoelastischen Anteils; im Überlappungsbereich ist eine Zuordnung zu einem der Prozesse auf experimentellem Wege nicht zweifelsfrei möglich.

ab. Dementsprechend wird im folgenden nur noch von „dem Atom“ und „dem Molekül“ gesprochen. Mit „Energieübertrag“ ist immer die in die innere Energie des Moleküls transferierte Energie gemeint.

In einem experimentellen Aufbau, der dem hier verwendeten ähnelt, wurde von HERTEL ET AL. die Streuung von $\mathrm{Na}(3 \mathrm{P})$ an $\mathrm{N}_{2}$ [33, 65, 32], $\mathrm{H}_{2}$ und $\mathrm{D}_{2}$ [67], $\mathrm{CO}$ [66], $\mathrm{O}_{2}$ sowie $\mathrm{NO}$ und einigen organischen Molekülen [34] untersucht. Mit den Ergebnissen für $\mathrm{Na}(3 \mathrm{P})-\mathrm{SiF}_{4}$ lassen sich am ehesten die Experimente an organischen Molekülen in [66] vergleichen; als Stoßpartner wurden dabei kleinere Alkene und Alkine verwendet, die einen verhältnismäßig großen Querschnitt für den diskutierten Prozeß aufweisen. Der Vergleich mit dem auch hier verwendeten statistischen Modell zeigt für die meisten dieser Moleküle einen Energieübertrag, der unter dem erwarteten liegt. Dies wird darauf zurückgeführt, daß nicht alle denkbaren Freiheitsgrade in den Stoßprozess involviert sind. Für das System Na(3P)-CO wird eine gute und für $\mathrm{Na}(3 \mathrm{P})-\mathrm{N}_{2}$ eine annehmbare Übereinstimmung mit den entsprechenden a prioriVerteilungen gefunden [33]. 
Für einige Systeme mit einem zweiatomigen Molekül als Stoßpartner liegen sowohl doppelt-differentielle Querschnitte über einen großen Winkelbereich als auch quantenchemisch berechnete Potentialflächen vor. Für das System Na(3P)-CO lassen sich dadurch Bereiche der Winkelverteilung einem über einen Komplex ablaufenden und andere einem direkten Mechanismus zuordnen [66]; für $\mathrm{Na}(3 \mathrm{P})-\mathrm{H}_{2}, \mathrm{D}_{2}$ ergibt sich ein direkter Mechanismus.

In [74] sind Experimente dargestellt, die im Gegensatz zu den bisher angegebenen das Molekül detektieren. Daher ist auch die Messung resonanten Energietransfers möglich, der jedoch für die Systeme $\mathrm{Na}(3 \mathrm{P})-\mathrm{N}_{2}, \mathrm{O}_{2}, \mathrm{CO}$ und $\mathrm{NO}$ nicht vorgefunden wird. Für $\mathrm{N}_{2}, \mathrm{O}_{2}$ und $\mathrm{CO}$ wird ein Energieübertrag gefunden, der geringer als der statistisch vorhergesagte ist; gleichzeitig neigen diese System zu Vorwärtsstreuung. Im Gegensatz dazu zeigt NO eine vorwärts/rückwärts-symmetrische Winkelverteilung und einen Energieübertrag, der etwas über dem statistisch vorhergesagten liegt. Insgesamt wird daraus gefolgert, daß $(\mathrm{NaNO})^{*}$ einen Stoßkomplex bildet, die anderen Systeme hingegen nicht. Diese Argumentation entspricht der im vorigen Abschnitt für $\mathrm{SiF}_{4}$ angegebenen.

Insgesamt ergeben die dargestellten Experimente keine Anhaltspunkte, die Annahme eines Stoßkomplexes für $\mathrm{Na}_{-} \mathrm{SiF}_{4}$ abzulehnen. Ebenso zeigt sich keine Evidenz für einen ausgezeichneten resonanten Energietransfer. 


\section{Kapitel 7}

\section{Streuung von $\mathrm{NH}_{3}$ an $\mathrm{Na}(3 \mathrm{~S})$ und $\mathrm{Na}(3 \mathrm{P})$}

\subsection{Motivation}

Zusätzlich zu den im Zentrum des Interesses stehenden Experimenten an $\mathrm{SF}_{6}$ und $\mathrm{SiF}_{4}$ wurde die Streuung von $\mathrm{NH}_{3}$ an Natrium untersucht. Motivation waren dabei vorläufige Ergebnisse quantenchemischer Rechnungen, die für das Molekül $\mathrm{Na}^{-\mathrm{NH}_{3}}$ im Grundzustand ein repulsives und ein schwach bindendes Potential im ersten elektronisch angeregten Zustand ergeben [73]. Dieses wird gestützt durch pump-probe-Experimente an $\mathrm{Na}\left(\mathrm{NH}_{3}\right)_{n}$ [72], die eine verhältnismäßig große Lebensdauer $(>1 n s)$ für den ersten elektronisch angeregten $\mathrm{Zu}-$ stand $\left(\tilde{A}^{2} E\right)$ von $\mathrm{NaNH}_{3}$ ergeben. Daraus entstand die Vermutung, zwischen der Streuung von $\mathrm{NH}_{3}$ an $\mathrm{Na}(3 \mathrm{~S})$ und $\mathrm{Na}(3 \mathrm{P})$ könnte sich eine deutliche Änderung im Verhalten ergeben. Die an diesem Streusystem gewonnenen Ergebnisse sollen hier ohne tiefergehenden Interpretationsanspruch dargestellt werden.

\subsection{Mögliche Ausgangskanäle}

Die Reaktion

$$
\mathrm{Na}+\mathrm{NH}_{3} \rightarrow \mathrm{NaH}+\mathrm{NH}_{2}
$$

ist endotherm mit einer Reaktionsenthalpie von $\Delta H=+180 \frac{\mathrm{KJ}}{\mathrm{mol}}$ entsprechend $1.87 \mathrm{eV}$ [10]. Damit ist der reaktive Kanal für die Streuung an $\mathrm{Na}(3 \mathrm{~S})$ nicht erreichbar, für die Streuung an $\mathrm{Na}(3 \mathrm{P})$ hingegen schon. Weiterhin denkbar ist die Kondensationsreaktion

$$
\mathrm{Na}+\mathrm{NH}_{3} \rightarrow \mathrm{NaNH}_{3},
$$

sofern der schwach gebundene Komplex nicht durch die Überschussenergie zerstört wird. Jedenfalls wurden $\mathrm{NaNH}_{3}$-Komplexe in massenspektrometrischen Untersuchungen [72] beobachtet. Für die Streuung an $\mathrm{Na}(3 \mathrm{P})$ ist außerdem ein Löschprozess entsprechend dem System Na-SiF 4 (Kap. 6) möglich, bei dem die Anregungsenergie des Natriums in Vibrationsenergie des $\mathrm{NH}_{3}$ überführt wird. 


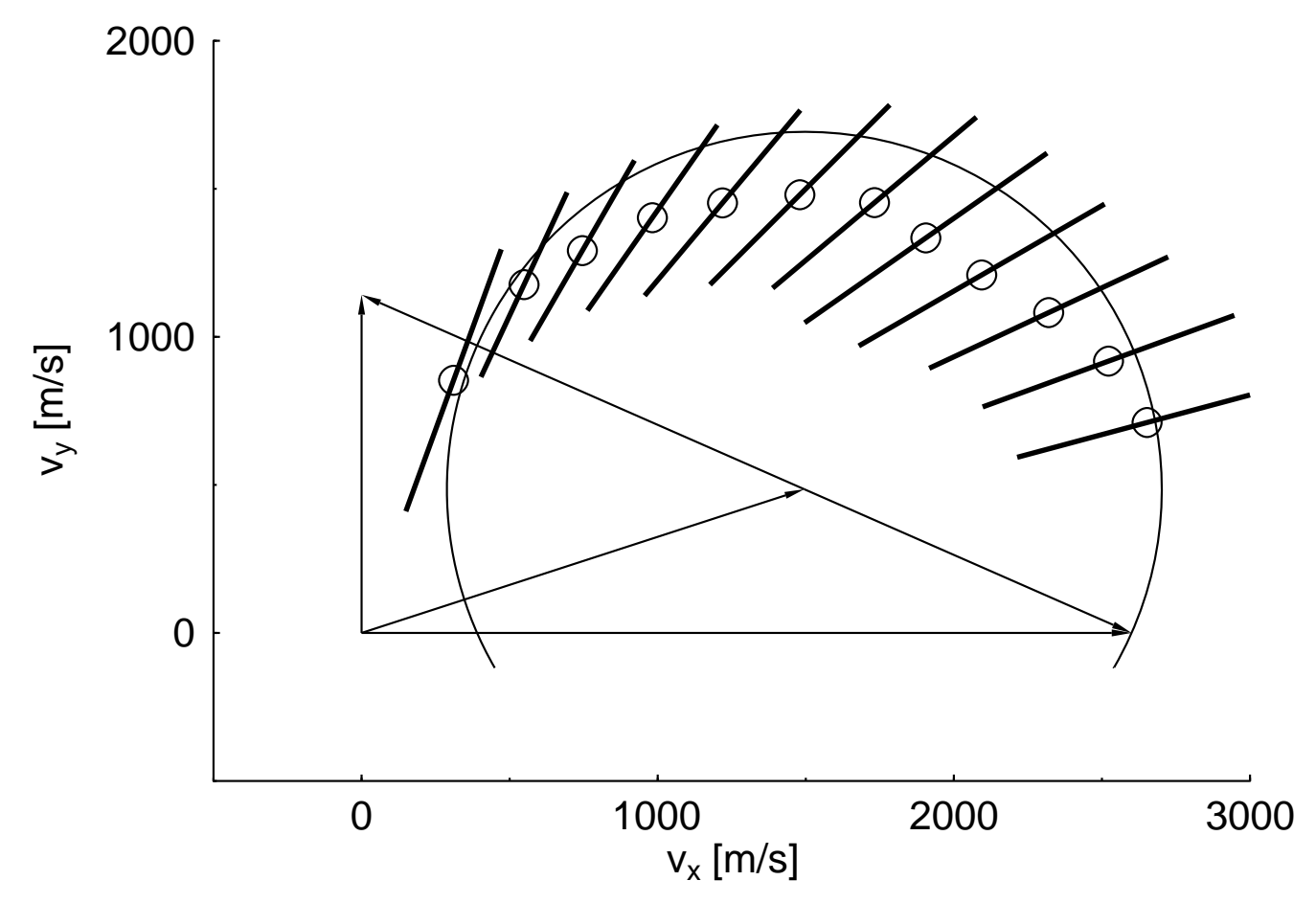

Abb. 7.1: Newton-Diagramm für die Streuung im Grundzustand.

\subsection{Experimentelle Bedingungen}

Die Messungen an $\mathrm{Na}(3 \mathrm{~S})-\mathrm{NH}_{3}$ und $\mathrm{Na}(3 \mathrm{P})-\mathrm{NH}_{3}$ wurden bei einer Natriumstrahlgeschwindigkeit von $2600 \frac{\mathrm{m}}{\mathrm{s}}$ bei einer Halbwertsbreite von ca. $300 \frac{\mathrm{m}}{\mathrm{s}}$ ausgeführt. Für die Geschwindigkeit des Ammoniak-Strahls ergibt sich aus Formel 2.11 bei einem Wert von $c_{p}^{0}=35.6 \frac{\mathrm{J}}{\mathrm{mol} \cdot \mathrm{K}}$ eine Geschwindigkeit von $1142 \frac{\mathrm{m}}{\mathrm{s}}$. Die Düse befand sich dabei auf Raumtemperatur. Insgesamt folgt daraus eine Stoßenergie im Schwerpunktsystem von $0.41 \mathrm{eV}$.

Die Laseranregung des Natriums wurde unter denselben Bedingungen ausgeführt wie im vorigen Kapitel bei der Streuung an $\mathrm{SiF}_{4}$. Demgemäß betrug die experimentelle Anregungseffizienz $15 \%$.

\subsection{Ergebnisse}

\section{Streuung im Grundzustand}

Für die Streuung von $\mathrm{Na}(3 \mathrm{~S})$ an $\mathrm{NH}_{3}$ wird ausschließlich elastische Streuung beobachtet. Die im Bereich mittlerer Winkel auftretende Abweichung zu elastischer Streuung im NewtonDiagramm (Abb. 7.1) kann nicht als signifikant angesehen werden, da eine verhältnismäßig 


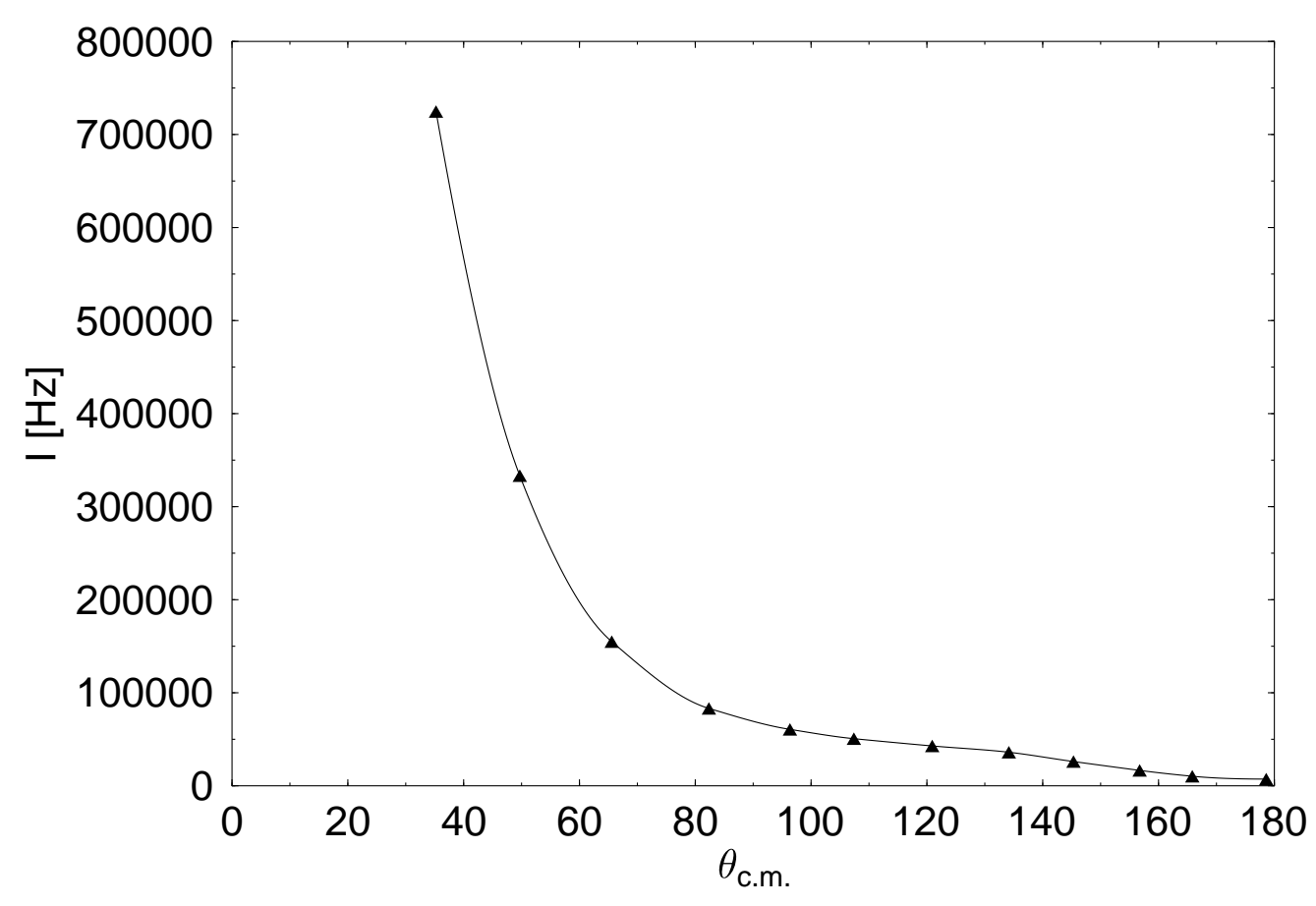

Abb. 7.2: Winkelverteilung der elastischen Streuung von $\mathrm{Na}(3 \mathrm{~S})$ an $\mathrm{NH}_{3}$.

große Unsicherheit in der berechneten Sekundärstrahlgeschwindigkeit vorliegt (s. Kap. 2.5). Im Gegensatz zu den in den vorigen Kapiteln vorgestellten Systemen ist das Newton-Diagramm symmetrischer, so daß sich ein Fehler in der Sekundärstrahlgeschwindigkeit stärker auf das Newton-Diagramm auswirkt. Die Winkelverteilung im Schwerpunktsystem zeigt Abb. 7.2.

\section{Streuung im angeregten Zustand}

Für die Streuung von $\mathrm{Na}(3 \mathrm{P})$ an $\mathrm{NH}_{3}$ wird für kleine Winkel in den Differenzspektren nur ein Beitrag elastischer Streuung beobachtet. Der Streuquerschnitt ist dabei grundsätzlich etwas größer als im Grundzustand. $\mathrm{Ab} \Theta_{L a b}=35^{\circ}$ zeigt sich ein weiterer Anteil im Flugzeitspektrum (s. Abb. 7.3). Die Auftragung dieses Anteils, der sich in den Flugzeitspektren bis $\Theta_{L a b}=70^{\circ}$ zeigt (Abb. 7.5), im Newton-Diagramm (Abb. 7.4) führt zu einer Zuordnung zu ausschließlicher Rückwärtsstreuung.

Messungen mit dem LIF-Detektor zeigen diesen Anteil nicht; allerdings weisen die mit dem LIF-Detektor aufgenommenen Spektren aufgrund der geringen Signalintensität erhebliches Rauschen auf. Damit läßt sich der rückwärts gestreute Anteil reaktiver Streuung zuordnen. Die Kondensationsreaktion 7.2 kann nicht Ursache für diesen Beitrag sein, da die Produkte dieser Reaktion auf den Schwerpunktswinkel des Systems gestreut werden müßten.

$\mathrm{Da}$ die in der Auswertung und Transformation ins Schwerpunktsystem verwendeten 


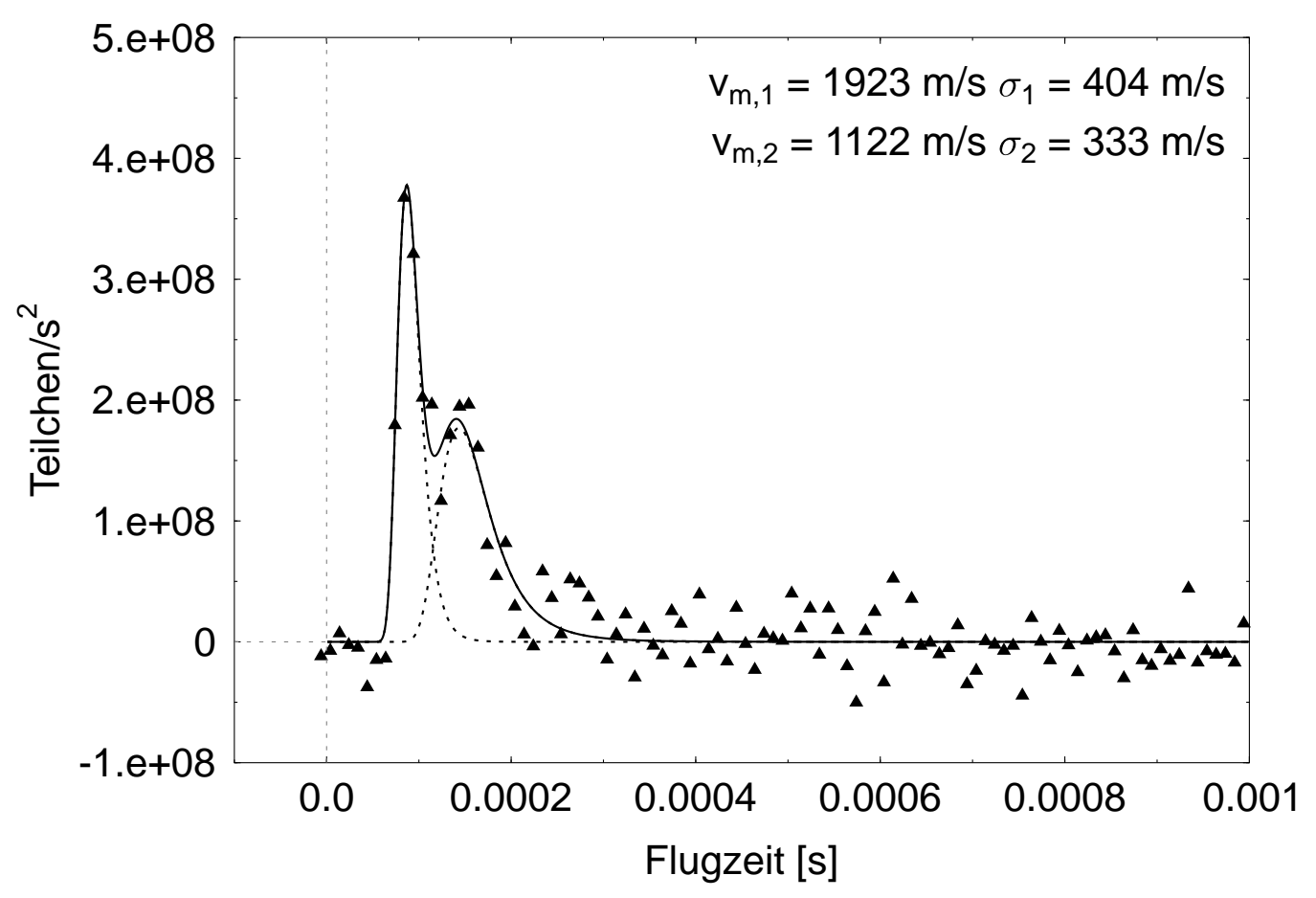

Abb. 7.3: Flugzeitspektrum $\mathrm{Na}(3 \mathrm{P})-\mathrm{NH}_{3}$ bei $\Theta_{L a b}=50^{\circ}$. Für die erste angepaßte transformierte Gauß-Funktion wurden Position und Breite aus dem entsprechenden Grundzustandsspektrum entnommen. Die Amplitude beschreibt die Differenz zwischen dem Querschnitt im Grund- und angeregten Zustand. Die zweite Funktion beschreibt den Anteil, der der Rückwärtsstreuung im Newton-Diagramm zugeordnet wird. Für größere Laborwinkel wandern die Anteile aufeinander zu.

Näherungen (s. Abschnitt 4.2.2 für den rückwärts gestreuten Anteil nicht unbedingt korrekt sind, wurde eine Simulation für reine Rückwärtsstreuung durchgeführt (analog $\mathrm{Ab}$ schnitt 4.3.2). Das Ergebnis (Abb. 7.6) ist mit der Lage der Flugzeitspektren im NewtonDiagramm konsistent. Für den rückwärts gestreuten Anteil ist die üblicherweise gewählte Auftragung $I_{C M} \leftrightarrow \Theta_{C M}$ nicht sinnvoll, da die Maxima aller Flugzeitspektren $\Theta_{C M}=$ $180^{\circ}$ zugeordnet werden. Daher wurde eine Auftragung gegen den Laborwinkel gewählt (Abb. 7.5). Die Änderung des Laborwinkels bewirkt in diesem Falle eine Änderung der Geschwindigkeit im Schwerpunktsystem, während die Winkelverteilung im Flugzeitspektrum abgelesen werden kann.

Unter der Annahme, daß tatsächlich $\mathrm{NaH}$ entsteht, ergibt sich folgende Energiebilanz : Im Stoß steht eine Energie von $0.42 \mathrm{eV}$ Stoßenergie und 2.1 eV Anregungsenergie des Natriums zur Verfügung. Es wird davon ausgegangen, daß sich die $\mathrm{NH}_{3}$-Moleküle im Vibrationsgrundzustand befinden und die innere Energie des $\mathrm{NH}_{3}$ damit keine Rolle spielt. Für den Ausgangskanal ergibt sich dann eine mittlere Schwerpunktsgeschwindigkeit von $1050 \frac{\mathrm{m}}{\mathrm{s}}$ für 


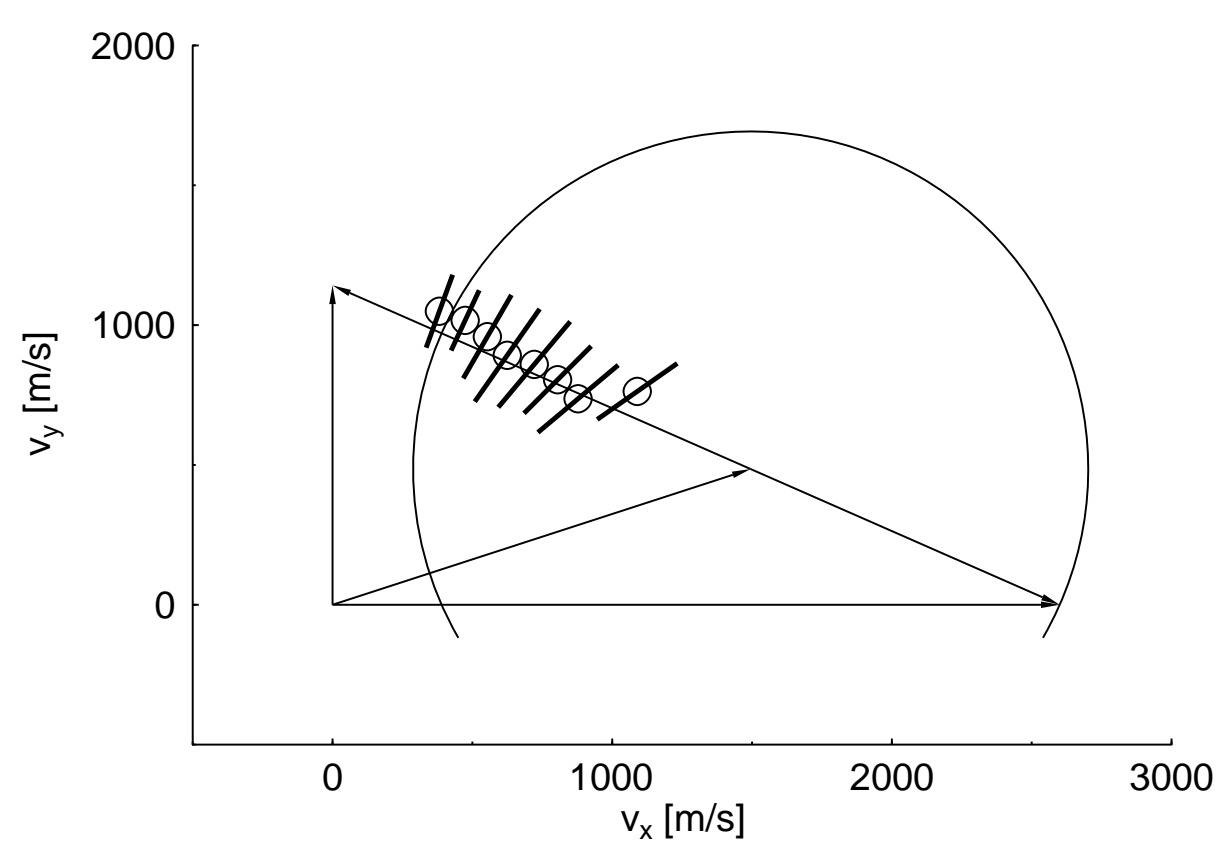

Abb. 7.4: Newton-Diagramm für den reaktiven Anteil der Streuung $\mathrm{Na}(3 \mathrm{P})-\mathrm{NH}_{3}$.

das $\mathrm{NaH}$ und $1891 \frac{\mathrm{m}}{\mathrm{s}}$ für das $\mathrm{NH}_{2}$. Mit der oben angegebenen Endothermizität folgt daraus eine kinetische Energie der Produkte von $0.42 \mathrm{eV}$ und damit eine gesamte innere Energie der Produkte von $0.23 \mathrm{eV}$.

Als Vergleich für das hier betrachtete System bietet sich das Streusystem $\mathrm{Na}-\mathrm{H}_{2}$ an, für das diverse Untersuchungen vorliegen. Im Gegensatz $\mathrm{zu} N a(3 P)-\mathrm{NH}_{3}$ ist jedoch die Reaktion zu $\mathrm{NaH}$ für $\mathrm{Na}(3 \mathrm{P})-\mathrm{H}_{2}$ energetisch nicht möglich [57]. Infolgedessen wird in einem dem hier vorgestellten entsprechenden Experiment an $\mathrm{Na}(3 \mathrm{P})-\mathrm{H}_{2}$ [67] keine Reaktion, sondern ein Löschprozess beobachtet. Vibrationsanregung des $H_{2}$ führt jedoch zu ausreichender Energie, so daß die Reaktion zu NaH tatsächlich beobachtet wird [57]. 


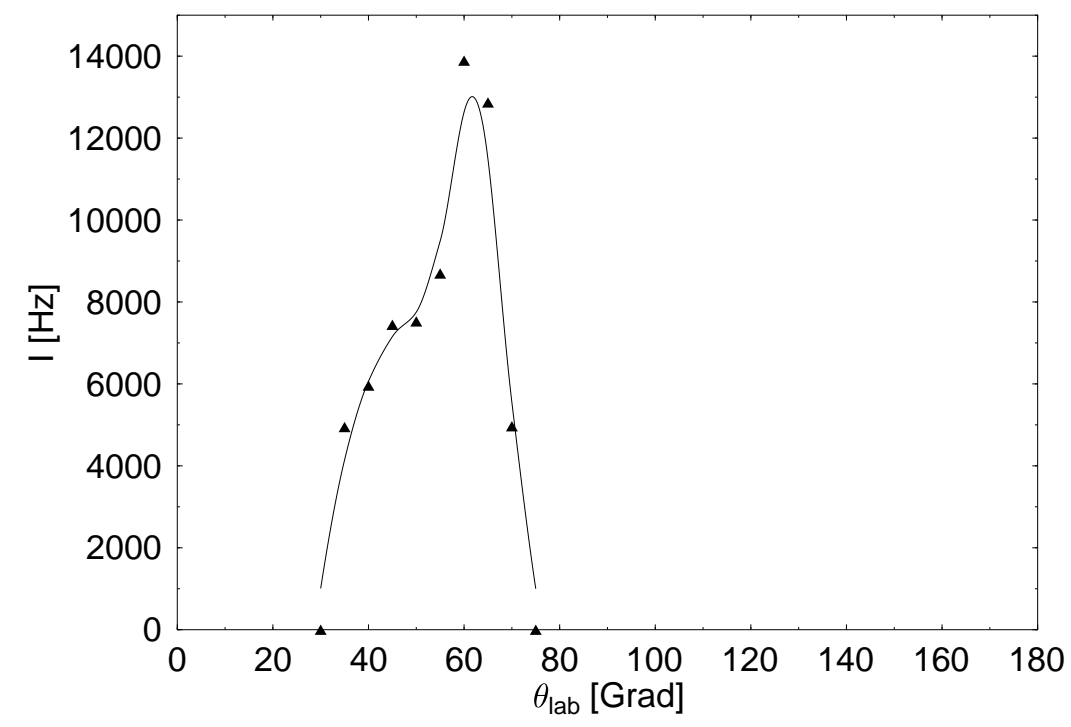

Abb. 7.5: Integrierte Intensität der Flugzeitspektren für den reaktiven Anteil in Abhängigkeit vom Laborwinkel. 


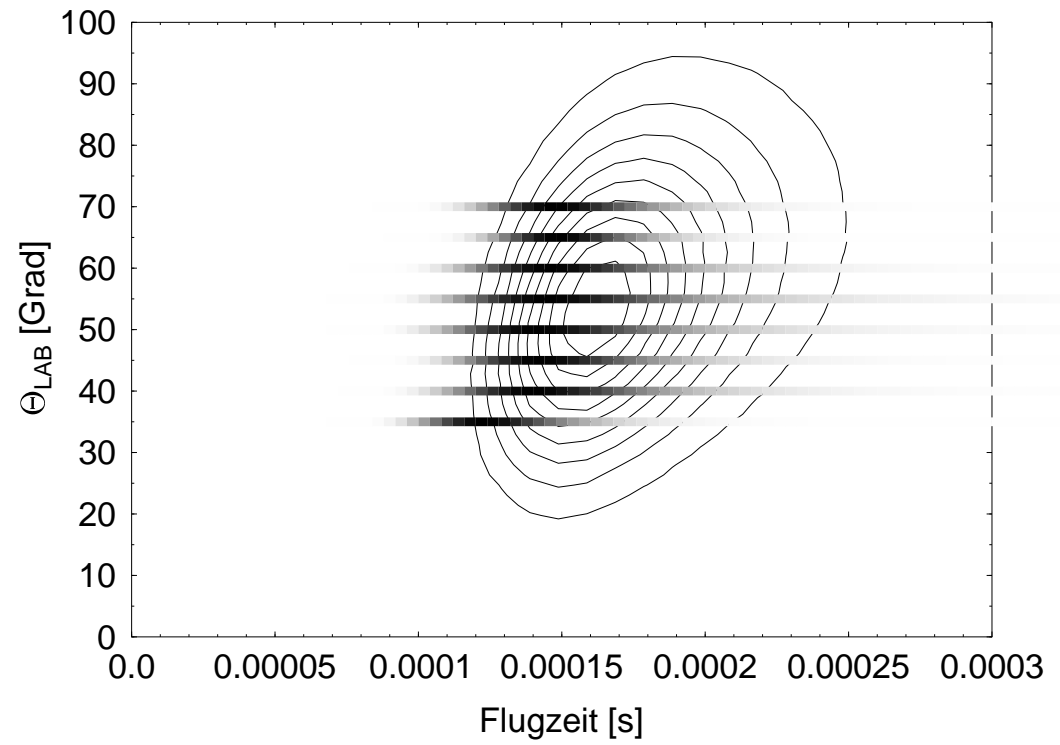

Abb. 7.6: Vergleich einer Simulation der Kinematik für reine Rückwärtsstreuung (Höhenlinien, $10 \%$ bis $90 \%$ des Maximalwertes) mit dem reaktiven Anteil aus den Flugzeitspektren (codiert in Grauwerten) im Laborsystem. Die Simulation belegt, daß sich Rückwärtsstreuung für die betrachtete Situation in den beobachteten Flugzeitverteilungen äußert. 


\section{Kapitel 8}

\section{Zusammenfassung}

Thema der vorliegenden Arbeit ist die Streuung von $\mathrm{Na}$ im Grundzustand und im ersten elektronisch angeregten Zustand (3P) an mehratomigen Molekülen. Dazu wurden Experimente mit der Methode der gekreuzten Molekularstrahlen durchgeführt. Gemessen wurden doppelt differentielle Streuquerschnitte, d. h. es wurde die gestreute Intensität in Abhängigkeit von Winkel und Geschwindigkeit der Teilchen ermittelt. Diese Messungen wurden für den Grundzustand des Natriums und für die Differenz zwischen angeregtem und Grundzustand aufgenommen. Aus diesen Messungen wurden die jeweiligen Streuquerschnitte für die Wechselwirkung mit $\mathrm{Na}(3 \mathrm{~S})$ und $\mathrm{Na}(3 \mathrm{P})$ bestimmt.

Für die Wechselwirkung zwischen Natrium und $\mathrm{SF}_{6}$ konnte nachgewiesen werden, daß die nichtreaktive Streuung auf großen Ablenkwinkeln von inelastischen Prozessen dominiert wird. Es findet ein Übertrag von bis zu $60 \%$ der verfügbaren Translationsenergie in die innere Energie des $\mathrm{SF}_{6}$ statt.

Die reaktive Streuung zeigt im Grundzustand eine winkelabhängige Geschwindigkeitsverteilung des nachgewiesenen Produktes $(\mathrm{NaF})$ : Für Vorwärts- und Rückwärtsstreuung wird eine größere Geschwindigkeit beobachtet als für dazwischenliegende Streuwinkel. Die Winkelverteilung der Produkte zeigt ein Maximum in Vorwärts- und ein niedrigeres Maximum in Rückwärtsrichtung. Mit zunehmender Stoßenergie wird die Verteilung symmetrischer. Zwischen den beiden Extrema zeigt sich eine konstante Winkelverteilung. Das Verhalten wird derart interpretiert, daß die Reaktion zwischen $\mathrm{Na}_{\mathrm{and}} \mathrm{SF}_{6}$ einen Grenzfall zwischen der Bildung eines Stoßkomplexes und einer direkten Reaktion darstellt. Für die reaktive Streuung an $\mathrm{Na}(3 \mathrm{P})$ sind die Produkte deutlich schneller als für die reaktive Streuung im Grundzustand. Eine deutliche Asymmetrie der Verteilung der Geschwindigkeiten mit dem Winkel zeigt sich nicht. Die Winkelverteilungen ähneln im Verhalten den für den Grundzustand beobachteten. Für die Zunahme des Reaktionsquerschnittes mit der Anregung ergibt sich eine fallende Tendenz mit der Stoßenergie. Sowohl für den Grundzustand als auch für den angeregten Zustand wird die verfügbare Energie zum größten Teil in die innere Energie der Produkte transferiert.

Zusammen mit den in [55] dargestellten Messungen liegen nun umfangreiche experimentelle Ergebnisse vor. Wünschenswert ist jetzt eine adäquate theoretische Betrachtung des Systems.

Das System $\mathrm{Na}-\mathrm{SiF}_{4}$ zeigt weder im Grundzustand noch im angeregten Zustand des Natriums reaktive Streuung. Für den Grundzustand wird ausschließlich elastische bzw. für 


\section{4}

größere Ablenkwinkel inelastische Streuung beobachtet. Die Streuung im angeregten $\mathrm{Zu}-$ stand zeigt zusätzlich zu dem im Grundzustand beobachteten Prozeß noch einen Anteil gestreuten Natriums, der eine deutlich geringere kinetische Energie aufweist. Dieser Anteil wird auf einen Löschprozess (,quenching“) zurückgeführt, also die Abregung des Natriums im Stoß mit gleichzeitigen Energieübertrag auf das $\mathrm{SiF}_{4}$. Der Energieübertrag wird durch ein einfaches statistisches Modell gut wiedergegeben. Daraus ergibt sich die Interpretation der Dynamik des Prozesses als Bildung eines langlebigen Stoßkomplexes.

Für das System $\mathrm{Na}-\mathrm{NH}_{3}$ wird im Grundzustand elastische Streuung beobachtet. Befindet sich das Natrium im angeregten Zustand, wird eine Reaktion beobachtet. Die nachweisbaren Produkte werden ausschließlich in Rückwärtsrichtung gestreut. Daraus und aus energetischen Überlegungen folgt, daß das Produkt Natriumhydrid sein muß und weiterhin, daß es sich bei der Reaktion um einen direkten Prozeß handelt.

Die in dieser Arbeit untersuchten Systeme habe im Gegensatz zu vielen bisher mit der Molekularstrahltechnik untersuchten als gemeinsames Merkmal, daß es sich um verhältnismäßig große Moleküle handelt. Dadurch liegt den Untersuchungen die verallgemeinerte Fragestellung nach dem Einfluß vieler Freiheitsgrade auf die Reaktionsdynamik zugrunde. Es zeigt sich deutlich verschiedenes Verhalten bezüglich der elektronischen Anregung des atomaren Stoßpartners. Als Gemeinsamkeit läßt sich feststellen, daß ein großer Teil der zur Verfügung stehenden Energie in die Vibrations- und Rotationsfreiheitsgrade des Systems übertragen wird. Außerdem zeigt sich eine indirekte Rückwirkung dieser Eigenschaft auf die Reaktivität. Wenn auch angesichts der Vielfalt möglicher Targetmoleküle eine Verallgemeinerung nicht offensichtlich ist, läßt sich abschließend feststellen, daß auch diese Systeme experimentellen Untersuchungen mit exakten Methoden zugänglich sind, die detaillierte Aussagen ermöglichen. 


\section{Literaturverzeichnis}

[1] A.G.Suits, P. De Pujo, O. Sublemontier, J.-P. Visticot, J. Berlande, J. Cuvellier, T. Gustavsson, J.-M. Mestdagh, P. Meynadier und Y. T. Lee: The dynamics of electronic to vibrational, rotational, and translational energy transfer in collision of Ba $\left({ }^{1} P_{1}\right)$ with diatomic. J. Chem. Phys., 97(6):4094, 1992.

[2] Alagia, Michele, Nadia Balucani, Piergiorgio Casavecchia, DomenioCO Stranges und Gian Gualberto VolPi: Reactive Scattering of Atoms and Radicals. J. CHEM. SOC. FARADAY TRANS., 91(4):575, 1995.

[3] Anderson, K., M. P. Folscher, R. Lindh, P. A. Malmquist, J. Olsen, B. O. RoOS, A. J. SADLEY und P. O. WIDMARK: MOLCAS version 2. University of Lund and IBM Sweden, 1991.

[4] BASsI, D.: Ionisation Detectors, Band 1 der Reihe Atomic and Molecular Beam Methods, Kapitel 7. G. Scoles, 1988.

[5] BECKORD, Klaus: Bau eines elektrooptischen Resonanzmodulators zur vollständigen Spinpolarisation eines Natrium-Atomstrahls durch optisches Pumpen auf zwei Frequenzen. Diplomarbeit, Universität Bielefeld, Mai 1989.

[6] Behrens, R., R. R. Herm und C. M. Sholeen: Product magnetic deflection slotted disk velocity analysis molecular beams kinetics: $\mathrm{Li}+\mathrm{SnCl}_{4}, \mathrm{PCl}_{3}$, and $\mathrm{SF}_{6}$. J. Chem. Phys., 65(11):4791, 1976.

[7] Boschetti, A., M. Zen, D. BAssi und M. Scotoni: Thermal effects in collisionfree infrared multiphoton absorption by SF6 and CF3Br. Chem. Phys. Lett., 87:131, 1984.

[8] BUCK, U.: General Principals and Methods, Band 1 der Reihe Atomic and Molecular Beam Methods, Kapitel 18. G. Scoles, 1988.

[9] Buck, U., F. Gestermann und H. Pauly: Messungen differentieller Streuquerschnitte bei Stößen von Na-Atomen mit mehratomigen Molekülen, Band 109. MPI für Strömungsforschung, 1975.

[10] Chase, M. W., C. A. Davies, J. R. Downey, D. J. Frurip, R. A-. McDonald und A. N. SYveruD: JANAF Thermochemical Tables, Third Edition. J. Phys. Chem. Ref. Data, 14(1), 1985. 
[11] ChEn, EDWARD C. H.: The nagative ion states of sulfur hexafluoride. J. Chem. Phys., 88(8):4711, 1988.

[12] DemtröDER, W.: Laser Spectroscopy. Springer-Verlag Berlin Heidelberg New York, 2 Auflage, 1982.

[13] DÜREN, R.: Some aspects of the averaging effects in measured differential cross sections, Band 102. MPI für Strömungsforschung, 1975.

[14] Düren, R., M. Färber, B. Heumann, M. Knepper, S. Mohr, C. Weiss, S. TE Lintel HeKkert, A. F. Linskens und J. Reuss: Differential cross sections for state specific reactive scattering of $\mathrm{Na}-\mathrm{SF}_{6} \rightarrow \mathrm{NaF}-\mathrm{SF}_{5}$. J. Chem. Phys., 104(10):3620, 1996.

[15] Düren, R., W. GRÖGER und R. LiedtKe: Pseudostatistical light chopper for timeof-flight analysis of laser excited species. Rev. Sci. Insrum., 56(377), 1985.

[16] DÜREN, R. und H.O. HOPPE: Mesurement and evaluation of differential cross sections for $\mathrm{Na}^{2} \mathrm{P}_{3}{ }_{2}$ with Hg. J. Phys. B: At. Mol. Phys., 11:2143, 1978.

[17] Düren, R., M. Knepper, A. Linskens, S. Milosevic, S. Mohr und H.-J. WALDAPFEL: Differential Cross Sections for Reactive Scattering of Na from $\mathrm{SF}_{6}$. J. Phys. Chem., 97(10):2059, 1993.

[18] Düren, R., M. Knepper, S. Mohr, S.Te Lintel HeKkert, A. F. Linskens und J. REUSS: Differential cross sections for elastic and reactive collisions between $\mathrm{Na}$ and multiple photon excited $S_{6}$. Chem. Phys. Lett., 41:228, 1994.

[19] DÜREN, R., U. LACKSCHEwITZ und S. MilošEvić: Reactive Scattering of Sodium and Hydrogen Fluoride on an Electronically Excited Surface. Chem. Phys., 126:81, 1988.

[20] Düren, R., U. LACKschewitz, S. MilošEvić, H. PANKnin und N. SchiRawski: Differential Cross Sections for Reactive and Non-Reactive Scattering of Electronically Excited Na from HF Molecules. Chem. Phys. Lett., 143(1):45, 1988.

[21] DÜren, R., U. Lackschewitz, S. Milošević und H.-J. WaldaPfEL: Scattering on Anisotropic Potential Energy Surfaces: $\mathrm{Na}\left(3^{2} \mathrm{~S}\right)$ and $\mathrm{Na}\left(3^{2} \mathrm{P}\right)$ with $\mathrm{H}_{2} \mathrm{O}$. Chem. Phys., 140:199, 1990.

[22] Eccles, J., G. Pfeffer, E. Piper, G. Ringer und J. P. Toennies: The Observation of Rotational and Vibrational Excitation of $S_{6}$ in the Scattering from Ar at center-of-mass Energies around $1 \mathrm{eV}$. Chem. Phys., 89:1, 1984.

[23] Eyal, M., F.R. Grabiner, U. AgAm und L.A. Gams: Vibrational and total energy dependence of the reaction rate of $\mathrm{Na}$ with $\mathrm{CO}$ laser-excited SF6. J. Chem. Phys., 75:4396, 1981. 
[24] Fischer, A. und I.V. HerTEL: Alignment and Orientation of the Hyperfine Levels of a Laser Excited Na-Atom Beam. Z. Phys. A, 304:103, 1982.

[25] Fluendy, M. A. D. und K. P. LAwley: Chemical Applications of Molecular Beam Scattering. Chapman and Hall, London, 1973.

[26] Fussen, D.: On the global efficiency of the laser optical excitation of a fast atomic beam. J. of Modern Optics, 38(9):1787, 1991.

[27] Gieler, M., F. Aumayr, R. Gaggl, C. Neureiter und L. Windholz: Application of an electrooptical modulator in inelastic collision studies with laser-excited Na(3p) atoms. J. Phys. B: At. Mol. Opt. Phys., 26:297, 1993.

[28] Goerke, A., G. Leipelt, H. Palm, C. P. Schulz und I. V. Hertel: Reactive Scattering of sodium clusters with molecular oxygen. Z. Phys. D., 32:311-320, 1995.

[29] Gonzalez-Urena, A. und R. Vetter: Reactive Collisions with Excited-state Atoms. J. CHEM. SOC. FARADAY TRANS., 91(3):389, 1995.

[30] Haken, H. und H. C. Wolf: Atom- und Quantenphysik. Springer-Verlag, 1980.

[31] Haken, H. und H. C. Wolf: Molekülphysik und Quantenchemie. Springer-Verlag, 1991.

[32] Hertel, I. V., H. Hofmann und K. A. Rost: Polarization Effects in the Differential Quenching Cross Sections of Na* by Diatomic Molecules. Phys. Rev. Lett., 38(7):343, 1977.

[33] Hertel, I. V., H. Hofmann und K. A. Rost: Crossed beam (E-VRT) energy transfer experiment. J. Chem. Phys., 71(2):674, 1979.

[34] HeRTEL, I. V. und W. ReILAND: Electronic to vibrational rotational energy transfer in collisions of $\mathrm{Na}\left(3^{2} \mathrm{P}\right)$ with NOand small organic molecules. J. Chem. Phys., 74(12):6757, 1981.

[35] Huber, K.P. und G. Herzberg: Molecular Spectra and Molecular Structure, Band IV. van Nostrand Reinhold Company, New York, 1979.

[36] Hubers, M. M. und J. Los: Ion pair formation in alkali-SF 6 collisions: dependence on collisional and vibrational energy. Chem. Phys., 10:235, 1975.

[37] HuISKEN, F.: Messung differentieller Wirkungsquerschnitte für die Rotationsanregung von $H D$-Molekülen im Stoß mit He und $D_{2}$. Doktorarbeit, MPI für Strömungsforschung, Göttinen, 1978.

[38] Huisken, F. und T. PERTSCH: CAMAC time-of-flight analyzer for molecular beam diagnostics. Rev. Sci. Instrum., 58(6):1038, 1987. 
[39] HüWEL, L.: Potentialbestimmung des Systems $\mathrm{Na}\left(3^{2} P\right)+H g\left(6^{1} S\right)$ durch Messung und Auswertung der Regenbogenstreuung im überthermischen Energiebereich von 0.7 bis $3.2 \mathrm{eV}$. Doktorarbeit, MPI f. Strömungsforschung, Göttingen, 1980.

[40] JR, R. P. Mariella, D. R. Herschbach und W. Klemperer: Molecular beam electric resonance spectra of reaction products: vibrational energy of LiF from Li and $S F_{6}$. J. Chem. Phys., 58(9):3785, 1973.

[41] Kappes, M., W. Kunz und E. Schumacher: Production of Large Sodium Clusters by Seeded Beam Expansions. Chem. Phys. Lett., 91(6):413, 1982.

[42] Kawano, H., S. ItAsaka und S. OHnishi: Time Variation of the Effective Work Function for Thermal Positive Ion Production from Lithium Atoms Incident upon a Rhenium Surface in a high Vacuum. Int. J. of Mass Spec. and Ion. Proc., 75:233-241, 1987.

[43] Kelly, J. F. und A. GALlGHER: Efficient electro-opticmodulator for optical pumping of Na beams. Rev. Sci. Instrum., 58(4):563-566, 1987.

[44] KNePPer, MARKo: Entwicklung und Anwendung eines Detektors zum spezifischen Nachweis gestreuter Natriumatome durch laserinduzierte Fluoreszenz. Diplomarbeit, MPI f. Strömungsforschung, Göttingen, 1993.

[45] LACKSCHEWITZ, U.: Molekularstrahlexperimente zur reaktiven und nichtreaktiven Streuung von $\mathrm{Na}\left(3^{2} \mathrm{P}_{\frac{3}{2}}\right.$ an Fluorwasserstoff. Doktorarbeit, MPI f. Strömungsforschung, Göttingen, 1988.

[46] LEE, Y. T.: Molecular Beam Studies of Elementary Chemical Processes. Science, 236:793, 1987.

[47] Leuken, J. J. van, J. Bulthuis, S. Stolte und H. J. Loesch: KBr Angular and Velocity Distributions from a Crossed Molecular Beam Study between K and Brute Force Oriented and Nonoriented $\mathrm{CH}_{3} B r$ Molecules. J. Phys. Chem., 99:13582-13590, 1995.

[48] Levine, R. D. und R. B. Bernstein: Molekulare Reaktionsdynamik. B. G. Teubner, Stuttgart, 1991.

[49] Liedenbaum, C., S. Stolte und J. Reuss: Multi-photon excitation of a beam of $\mathrm{SF}_{6}$ molecules pumped and probed by $\mathrm{cw}-\mathrm{CO}_{2}$ lasers. Chem. Phys., 122:443, 1988.

[50] Lijnes, P. L.: Review of Literature on Quenching, Excitation and Mixing Collision Cross Sections for the First Resonance of the Alkalis. Physics Laboratory of Utrecht, 1972.

[51] MeIJer, H. A. J.: Associative Ionisazion of Two Laser Excited Na Atoms. Doktorarbeit, Rijksuniversiteit te Utrecht, 1988. 
[52] Mestdagh, J.M., B.A. Balko, M.H. Covinsky, P.S. Weiss, M.F. Vernon, H. SChMidT und Y.T. LeE. Faraday Discuss. Chem. Soc., 84:145, 1987.

[53] Mestdagh, J. M., B. A. Balko, M. H. Covinsky, P. S. Weiss, M. F. Vernon, H. SCHMIDT und Y. T. LEE: Reactive Scattering of Electronically Excited Alkali-metal Atoms with Molekules. Faraday Discuss. Chem. Soc., 84:145, 1987.

[54] Miller, D. R.: Free Jet Sources, Band 1 der Reihe Atomic and Molecular Beam Methods, Kapitel 2. G. Scoles, 1988.

[55] MoнR, S.: Molekularstrahlexperimente zur reaktiven Streuung an vibrationsangeregten Molekülen. Doktorarbeit, MPI f. Strömungsforschung, Göttingen, 1995.

[56] MormignY, J. und R. Locht: The appearance of $\mathrm{CH}_{3}^{+}$ions from methyl halides by non-resonant photoionization. A translational energy surprisal analysis. Part I. $\mathrm{CH}_{3} \mathrm{~F}$. Chem. Phys., 206:225, 1996.

[57] Motzkus, M., G. Pichler, K. L. Kompa und P. HeRing: Local laser superheating due to quenching determined by degenerate four-wave-mixing and absorption thermometry. Chem. Phys. Lett., 257:181, 1996.

[58] Murell, J. N. und S. D. Bosanac: Introduction to the Theory of Atomic and Molecular Collisions. John Wiley \& Sons Ltd., 1989.

[59] Nijland, J. H., J. J. Blangé, H. Rudolf, H. A. Dijkerman und H. G. M. HeIDEMANN: Energy pooling reactions between two $\mathrm{Na}(3 \mathrm{p})$ atoms specified according to magnetic substate combinations. J. Phys. B, 25:4835, 1992.

[60] PACK, R. T.: van der Waals Coefficients for Interactions Involving Sulfur Hexafluoride. J. Chem. Phys., 86:2794, 1982.

[61] Pauly, H.: Other Low-energy Beam Sources, Band 1 der Reihe Atomic and Molecular Beam Methods, Kapitel 4. G. Scoles, 1988.

[62] Popov, V. K., M. Rossberg, W. Strube, J. Wollbrandt und E. Linke: LASER-INDUCED FLUORESCENCE SPECTROSCOPY OF SiF PRODUCED BY IR MULTIPLE-PHOTON DISSOCIATION OF SiF 4 . J. Mol. Struct., 218:225, 1990.

[63] Poppe, Dirk, Daniela Papierowska-Kaminsky und Vlasta Bonačić: The quenching of $\mathrm{Na} *(3 p)$ in collisions with N2: A classical trajectory study. J. Chem. Phys., 86(2):822, 1987.

[64] Press, W. H., B. P. Flannery, S. A. Teukolsky und W. T. Vetterling: Numerical Recipes. Cambridge University Press, Cambridge, 1st Auflage, 1986.

[65] Reiland, W., C. P. Schulz, H.-U. Tittes und I. V. Hertel: CROSSED BEAM STUDY OF THE Na $\left(3^{2} P_{3} / 2\right)+N_{2}$ QUENCHING PROCESS: ANGULAR DISTRIBUTIONS WITH PRODUCT STATE ANALYSIS. Chem. Phys. Lett., 91(5):329, 1982. 
[66] Reiland, W., H.-U. Tittes, I. V. Hertel, V. Bonacic-Koutcky und M. PerSICO: Stereochemical Effects in the Quenching of $N a^{*}\left(3^{2} P\right)$ by CO: Crossed Beam Experiment and ab initio CI Potential energy surfaces. J. Chem. Phys., 77(4):1908, 1982.

[67] Reiland, W., U. Tittes und I. V. Hertel: Angular Distributions for a Model System of Nonadiabatic Molecular Collisions: The Quenching of $\mathrm{Na}^{*}$ by $\mathrm{H}_{2}$ and $\mathrm{N}_{2}$. Phys. Rev. Lett., 48(20):1389, 1982.

[68] RILEY, S. J. und D. R. HERSCHBACH: Molecular beam kinetics: Long-lived collision complexes in reactions of $K, R b$, and $C s$ with $\mathrm{SnCl}_{4}$ and $\mathrm{SF}_{6}$. J. Chem. Phys., 58(1):27, 1973.

[69] Sanjurjo, A., K. M. Sancier, R. M. Emerson, M. Rustinioni, G. MarchetTI, D. GANDOLFI und G. Missoni: IMPROVEMENTS IN THE SiF $-\mathrm{Na}$ PROCESS. In: 7th E.C. Photovoltaic solar energy conference proceedings, Sevilla, 1986.

[70] SCHÄFER, K. und E. LAX (HRSG.): Landolt-Börnstein, 6. Auflage, Band II/2a. 1985.

[71] Schmidt, H., A. B ̈̈hring, E. Meyer und B. Miller: Preparation of Molecular States in an Ion-Atom Scattering Experiment by Laser Excitation. Phys. Rev. Lett., 48(15):1008, 1982.

[72] Schulz, C. P., J. Höhndorf, P. Brockhaus, F. Noack und I. V. Hertel: Ultrafast fragmentation of small alkali atom-ammonia clusters. Chem. Phys. Lett., 239:18, 1995.

[73] SCHulZ, S.: private Mitteilung. 1995.

[74] Silver, J. A., N. C. Blais und G. H. KwEI: Crossed beam studies of the dynamics of the electronic energy transfer: Quenching of $\mathrm{Na}\left(3 \mathrm{p}^{2} \mathrm{P}_{3} / 2\right)$ atoms by $\mathrm{N}_{2}, \mathrm{O}_{2}, \mathrm{CO}$, and NO molecules. J. Chem. Phys., 71(8), 1979.

[75] SISKA, P. E.: Iterative unfolding of intensity data, with application to molecular beam scattering. J. Chem. Phys., 59(11):6052, 1973.

[76] StromeIER, P.: A two-mode-laser as an excellent tool for exciting sodium atoms from both ground state levels simultaneously. Opt. Comm., 79(3):187, 1990.

[77] Verdasco, E., C.S. Rabanos, A. Ure na, F.J. Aoiz und V.J. Herrero: Molecular beam study of the radical group effect in the $\mathrm{K}+\mathrm{RI}$ to $\mathrm{KI}+\mathrm{R}\left(\mathrm{R}=\mathrm{CH}_{3}, \mathrm{C}_{2} \mathrm{H}_{5}\right.$, $n \mathrm{C}_{3} \mathrm{H}_{7}$ ) reactive collisions. Mol. Phys., 59(4):707, 1986.

[78] Verolainen, Ya. F. und A. Ya. Nikolaich: Radiative lifetimes of excited states of atoms. Sov. Phys. Usp., 25(6):431, 1982.

[79] WALDAPFEL, H.J.: Molekularstrahlexperimente zur reaktiven Streuung von Natrium an Schwefelhexafluorid. Doktorarbeit, MPI für Strömungsforschung, Göttingen, 1992. 
[80] WeISS, Claus: Anregung eines Atomstrahl mit einem Zwei-Farben-Laser für Streuexperimente. Diplomarbeit, MPI f. Strömungsforschung, Göttingen, 1996.

[81] Wilson, A. D. und R. D. Levine: Simple models of vibrational excitation in energy transfer molecular collisions. Mol. Phys., 27(5):1197, 1974.

[82] YARIV, AMnON: Introduction to Optical Electronics. Holt, Rinehard \& Winston, New York, 2 Auflage, 1976. 


\section{Herstellernachweis}

[1] Fa. Radiant Dyes, Wermelskirchen Niederdruckdüse RDSN 02

[2] Fa. Heraeus, Köln Rhenium $99.9 \%$.

[3] Fa. Leybold-Heraeus, Köln Quadrupol-Massenspektrometer Typ Quadruvac Q 200

[4] Fa. Neumüller, Hamburg Hochtemperatur-Heizleiter WBT 1,6

[5] Fa. Phillips Components, Hamburg Channel Electron Multiplier X719BL 


\section{Abbildungsverzeichnis}

2.1 Schema der Apparatur. . . . . . . . . . . . . . . . . . . . 6

2.2 Newton-Diagramm für den Fall inelastischer Streuung. . . . . . . . . . . . 15

2.3 Hyperfeinstrukturaufspaltung der Natrium-Übergänge. . . . . . . . . . . . 16

2.4 Dampfdruckkurve von Natrium, entnommen aus [70] . . . . . . . . . 16

3.1 Schnitt durch die Apparatur in Strahlebene . . . . . . . . . . . . . . . 18

3.2 Na-Ofen. . . . . . . . . . . . . . . . . . . . . . . . . . . . . . 19

3.3 Düse . . . . . . . . . . . . . . . . . . . . . . . . . . . . . . . 19

3.4 Intensität der Seitenbänder . . . . . . . . . . . . . . . . . . . 23

3.5 Gemessenes Frequenzspektrum hinter dem EOM . . . . . . . . . . . . . 24

3.6 Technischer Aufbau des EOM . . . . . . . . . . . . . . . . . . 25

3.7 Strahlführung innerhalb der Streukammer. . . . . . . . . . . . . . . . 27

3.8 Geschwindigkeitsmessung am Primärstrahl mit dem Dopplereffekt. . . . . 28

3.9 Schematischer Aufbau des LIF-Detektors. . . . . . . . . . . . . . . . . . 31

3.10 Scheibe des Pseudo-Random-Choppers . . . . . . . . . . . . 32

3.11 Zeitdiagramm . . . . . . . . . . . . . . . . . 34

4.1 Fluoreszenz in der Streuzone . . . . . . . . . . . . . . . . . . . . . 37

4.2 Zur Bestimmung von $\eta \ldots \ldots \ldots \ldots \ldots$

4.3 F-Besetzung nach der Anregung . . . . . . . . . . . . . . . . . . . . . . 39

4.4 Wesentliche geometrische Apparaturparameter. . . . . . . . . . . . . . 44

4.5 Newtondiagramm Na-Xe . . . . . . . . . . . . . . . . . . . . . 45

4.6 Flugzeitspektrum Na-Xe auf $\theta_{l a b}=10^{\circ} \ldots \ldots \ldots \ldots$

4.7 Simuliertes Experiment für Streuung ausschließlich auf $\Theta_{c m}=20^{\circ} . \quad \ldots .48$

4.8 Kanalbreite im Geschwindigkeitsraum in Abhängigkeit von der Geschwindigkeit. . . . . . . . . . . . . . . . . . . 49

4.9 Flugzeitspektren für $\mathrm{Na}^{-\mathrm{SF}_{6}}$, versch. Kanalbreiten . . . . . . . . . . . . 51

$5.1 \quad$ Flugzeitspektrum bei $\Theta_{L A B}=20^{\circ} \ldots \ldots \ldots \ldots \ldots$

5.2 Differenz-Flugzeitspektrum bei $\Theta_{L A B}=20^{\circ} \ldots \ldots \ldots \ldots \ldots$

5.3 Flugzeitspektrum bei $\Theta_{L A B}=70^{\circ} \ldots \ldots \ldots \ldots \ldots$

5.4 Differenz-Flugzeitspektrum bei $\Theta_{L A B}=70^{\circ} \ldots \ldots \ldots \ldots$. . . . . . . 61

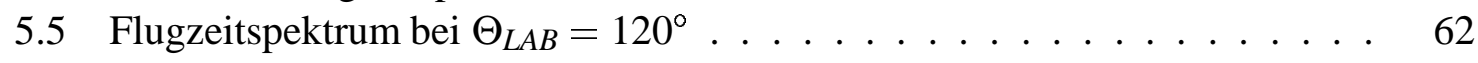

5.6 Differenz-Flugzeitspektrum bei $\Theta_{L A B}=120^{\circ} \ldots \ldots \ldots \ldots$

5.7 Auftragung des nichtreaktiven Anteils aus der Streuung von Na(3P) bzw. $\mathrm{Na}(3 \mathrm{P})$ an $\mathrm{SF}_{6}$ im Newton-Diagramm $(0.6 \mathrm{eV}) \quad \ldots \ldots \ldots \ldots$ 
5.8 Winkelverteilung des nichtreaktiven Anteils aus der Streuung von $\mathrm{Na}(3 \mathrm{~S})$ an $\mathrm{SF}_{6} \mathrm{im}$ Schwerpunktsystem. Die zugehörige Stoßenergie ist $0.6 \mathrm{eV}$. . . . .

5.9 Auftragung des reaktiven Anteils aus der Streuung von $\mathrm{Na}(3 \mathrm{~S})$ an $\mathrm{SF}_{6}$ im Newton-Diagramm. Die zugehörige Stoßenergie ist $0.6 \mathrm{eV}$. . . . . . . . 66

5.10 Winkelverteilung des reaktiven Anteils aus der Streuung von $\mathrm{Na}(3 \mathrm{~S})$ an $\mathrm{SF}_{6}$ im Schwerpunktsystem. Die zugehörige Stoßenergie ist $0.6 \mathrm{eV}$. . . . . . .

5.11 Auftragung des reaktiven Anteils aus der Streuung von $\mathrm{Na}(3 \mathrm{P})$ an $\mathrm{SF}_{6}$ im Newtondiagramm. Die zugehörige Stoßenergie ist $0.6 \mathrm{eV} . \quad \ldots \ldots \ldots$. . . .

5.12 Winkelverteilung des reaktiven Anteils aus der Streuung von $\mathrm{Na}(3 \mathrm{P})$ an $\mathrm{SF}_{6}$ im Schwerpunktsystem. Die zugehörige Stoßenergie ist $0.6 \mathrm{eV}$.

5.13 Auftragung des nichtreaktiven Anteils aus der Streuung von $\mathrm{Na} \mathrm{SF}_{6} \mathrm{im}$ Newton-Diagramm $(2.0 \mathrm{eV}) \ldots \ldots \ldots \ldots$

5.14 Winkelverteilung des nichtreaktiven Anteils aus der Streuung von $\mathrm{Na}(3 \mathrm{~S})$ an $\mathrm{SF}_{6}$ im Schwerpunktsystem. Die zugehörige Stoßenergie ist $2.0 \mathrm{eV}$. . . . .

5.15 Auftragung des reaktiven Anteils aus der Streuung von $\mathrm{Na}(3 \mathrm{~S})$ an $\mathrm{SF}_{6}$ im Newton-Diagramm. Die zugehörige Stoßenergie ist $2.0 \mathrm{eV}$. . . . . . . . .

5.16 Winkelverteilung des reaktiven Anteils aus der Streuung von $\mathrm{Na}(3 \mathrm{~S})$ an $\mathrm{SF}_{6}$ im Schwerpunktsystem. Die zugehörige Stoßenergie ist $2.0 \mathrm{eV}$. . . . . . .

5.17 Auftragung des reaktiven Anteils aus der Streuung von $\mathrm{Na}(3 \mathrm{P})$ an $\mathrm{SF}_{6}$ im Newtondiagramm. Die zugehörige Stoßenergie ist $2.0 \mathrm{eV}$. . . . . . . .

5.18 Winkelverteilung des reaktiven Anteils aus der Streuung von $\mathrm{Na}(3 \mathrm{P})$ an $\mathrm{SF}_{6}$ im Schwerpunktsystem. Die zugehörige Stoßenergie ist $2.0 \mathrm{eV}$. . . . . . 75

5.19 Auftragung der elastischen bzw. inelastischen Streuung von $\mathrm{Na}$ an $\mathrm{SF}_{6}$. . . 79

5.20 Anteil der in innere Energie des $\mathrm{SF}_{6}$ transferierten Stoßenergie. . . . . . . 80

5.21 Potentialfläche für den Grundzustand. . . . . . . . . . . . . . . 80

5.22 Potentialfläche für den ersten angeregten Zustand . . . . . . . . . . . 81

6.1 Newton-Diagramm $\mathrm{SiF}_{4} \ldots \ldots \ldots \ldots \ldots$. . . . . . . . . 84

6.2 Flugzeitspektrum $\mathrm{Na}(3 \mathrm{~S})-\mathrm{SiF}_{4} \ldots \ldots \ldots \ldots \ldots$

6.3 Flugzeitspektrum $\mathrm{Na}(3 \mathrm{P})-\mathrm{SiF}_{4} \ldots \ldots \ldots \ldots \ldots$

6.4 Winkelverteilungen $\mathrm{SiF}_{4} \ldots \ldots \ldots \ldots$

6.5 Energieverteilung $\mathrm{SiF}_{4} \ldots \ldots \ldots \ldots 1$

6.6 Flugzeitspektrum $\mathrm{Na}(3 \mathrm{P})-\mathrm{SiF}_{4}$, gefittet mit der a priori-Verteilung. . . . . . 92

7.1 Newton-Diagramm für die Streuung im Grundzustand. . . . . . . . . . . . 96

7.2 Winkelverteilung der elastischen Streuung von $\mathrm{Na}(3 \mathrm{~S})$ an $\mathrm{NH}_{3} \ldots \ldots \ldots$

7.3 Flugzeitspektrum $\mathrm{Na}(3 \mathrm{P})-\mathrm{NH}_{3}$ bei $\Theta_{L a b}=50^{\circ} \ldots \ldots \ldots \ldots$

7.4 Newton-Diagramm für den reaktiven Anteil der Streuung $\mathrm{Na}(3 \mathrm{P})-\mathrm{NH}_{3}$. . . 99

7.5 Integrierte Intensität der Flugzeitspektren für den reaktiven Anteil in Abhängigkeit vom Laborwinkel. . . . . . . . . . . . . . . . . . . . . . . . . 100

7.6 Vergleich einer Simulation der Kinematik für reine Rückwärtsstreuung mit dem reaktiven Anteil aus den Flugzeitspektren . . . . . . . . . . . . . 101 


\section{Anhang A}

\section{Eingesetzte Software}

Für die Ansteuerung des CAMAC-Systems und damit für die gesamte Apparatursteuerung und Meßwerterfassung wurden Programme aus eigener Entwicklung eingesetzt.

Die für die Auswertung der Daten und die Erstellung dieser Arbeit notwendigen Programme sind fast alle über das Internet frei verfügbar. Einige der Autoren bitten in Verbindung mit der Bereitstellung ihrer Programme um die Zitierung in mit deren Hilfe erstellten Publikationen; dem soll hier Genüge getan werden. Allen Autoren sei für die freie Veröffentlichung ihrer Programme gedankt.

Zum Anpassen der transformierten Gaussfunktionen an die Flugzeitspektren wurde FuDGIT von M. D. Lacasse verwendet. Dabei wurde ausgenutzt, daß FUDGIT einen relativ einfachen, interaktiven Zugang zu den der Anpassung von Funktionen dienenden Routinen von Numerical Recipes [64] bietet. Die Plotausgabe erfolgt dabei mittels GNUPLOT.

Die meisten graphischen Darstellungen wurden mit GLE von Chris Pugmire erzeugt.

Das Kinematik-Simulationsprogramm wurde unter GNU C++ erstellt; dabei wurde in erheblichem Maße von den mitgelieferten Bibliotheken Gebrauch gemacht.

Der Satz der Arbeit erfolgte mit $\mathrm{LT}_{\mathrm{E}} \mathrm{X}$; alle erwähnten Programme wurden unter dem frei verfügbaren Betriebssystem LINUX eingesetzt. 
118 


\section{Danksagung}

Die vorliegende Arbeit entstand in der Abteilung Atom- und Molekülphysik des Max-Planck-Instituts für Strömungsforschung unter Leitung von Herrn Prof. Dr. H. Pauly.

Besonders möchte ich mich bei Herrn Prof. Dr. R. Düren bedanken, der diese Arbeit betreut hat. Er hat in vielen Diskussionen den Gang der Experimente begleitet und zur Interpretation der Ergebnisse beigetragen.

Die meisten Messungen wurden gemeinsam mit Dipl.-Phys. Claus Weiß ausgeführt, dessen Diplomarbeit parallel zu dieser Arbeit entstanden ist. An einigen Messungen war auch Dr. S. te Lintel Hekkert beteiligt.

Für die Durchführung der Experimente war in erheblichem Maße technische Unterstützung notwendig. Für die vielen geleisteten Arbeiten sei hier den technischen Mitarbeitern W. Keiderling, R. Krohn, W. Kühn, G. Majewski und W. Stieler herzlich gedankt. Auch die Mitarbeiter und Mitarbeiterinnen der ServiceAbteilung des Instituts haben sich sehr entgegenkommend gezeigt.

Teilweise wurde diese Arbeit gefördert durch das EG-Netzwerk "Laser controlled dynamics". 